\title{
The homotopy exact sequence for the fundamental group scheme and infinitesimal equivalence relations
}

\author{
João Pedro dos Santos \\ Dedicated to the memory of Vikram Bhagvandas Mehta
}

\begin{abstract}
Let $f: X \rightarrow S$ be a morphism of smooth and connected schemes over an algebraically closed field $k$. What can be said about the relation between the three fundamental group schemes in sight: $\Pi(X), \Pi(S)$, and $\Pi($ Fibre)? This work addresses this question under the extra assumption that $f$ is smooth, projective, and geometrically connected. Moreover, it wishes to reach a natural answer by employing a method of proof analogous to the one presented by its precursor, Exposé X of [SGA1]. Our approach is then based on a new Tannakian criterion for studying exact sequences, and on infinitesimal equivalence relations. The latter objects are inspired by Ehresmann's image of connections on general fibre spaces. The proofs make no distinction between the cases $\operatorname{char}(k)=0$ and $\operatorname{char}(k)>0$.
\end{abstract}

\section{Introduction}

In this work we wish to convey a method and a means to prove the following.

TheOrem 1.1. Fix an algebraically closed field $k$. Let $X$ and $S$ be smooth and connected $k$ schemes. Let $f: X \rightarrow S$ be a projective and smooth morphism having geometrically connected fibres. Let $x_{0}$ be a $k$-point of $X$ and denote by $s_{0}$ its image in $S$.

(i) The homomorphism of fundamental group schemes $b: \Pi\left(X, x_{0}\right) \rightarrow \Pi\left(S, s_{0}\right)$ is faithfully flat.

(ii) If $X_{0}$ is the fibre of $f$ above $s_{0}$, then the image of $a: \Pi\left(X_{0}, x_{0}\right) \rightarrow \Pi\left(X, x_{0}\right)$ is precisely the kernel of $b$.

The fundamental group scheme $\Pi\left(X, x_{0}\right)$ alluded to in the statement is the one obtained by Tannakian duality [DM82] from the category of $\mathcal{D}_{X / k}$-modules which are $\mathcal{O}_{X}$-coherent [dSan07, 2.1.1]. Here, $\mathcal{D}_{X / k}$ is the ring of differential operators of $\left[\mathrm{EGA}, \mathrm{IV}_{4}, 16.8\right.$, p. 39ff] (which equals the expected one if $\operatorname{char}(k)=0)$. See $\S 6.1$ for more information concerning $\Pi$.

In [SGA1, X], the reader will find a result similar to that of Theorem 1.1, labelled the "homotopy exact sequence", where $\Pi(-,-)$ is replaced by Grothendieck's "algebraic fundamental group". Our strategy consists in following the natural transposition of the proof presented in

Received 19 March 2014, accepted in final form 18 February 2015.

2010 Mathematics Subject Classification 14F10, 14F35, 14L15.

Keywords: $\mathcal{D}$-modules, fundamental group schemes, formal schemes, connections on fibre bundles.

This journal is (C) Foundation Compositio Mathematica 2015. This article is distributed with Open Access under the terms of the Creative Commons Attribution Non-Commercial License, which permits non-commercial reuse, distribution, and reproduction in any medium, provided that the original work is properly cited. For commercial re-use, please contact the Foundation Compositio Mathematica. 


\section{J. P. DOS SANTOS}

[SGA1] and facing the inherent difficulties, including the immateriality of char $(k)$. There, since the quotient of a profinite group $\pi$ by an open subgroup $U$ still has a meaning in terms of finite $\pi$-sets, the most obvious set-theoretical criterion, [SGA1, V, Proposition 6.11, p. 114], makes perfect sense. The proof then makes use of this criterion and a method to "push forward" finite etale coverings [SGA1, X, 1.2, p. 202]. It is therefore reasonable to say that there are two main structures sustaining the "homotopy exact sequence". The understanding of these in the context of the fundamental group scheme is what motivates the present research.

As is well known, the theory of Tannakian categories meets an impediment in dealing with the fact that homogeneous spaces of algebraic groups are not necessarily affine. Although this difficulty is not found in the theory of [SGA1] (see the previous paragraph), in the problem we are tackling this is precisely what forces the introduction of new methods. In showing that $\Pi\left(S, s_{0}\right)$ is the cokernel of $a: \Pi\left(X_{0}, x_{0}\right) \rightarrow \Pi\left(X, x_{0}\right)$ we are led to understand properties of something which might not be an affine scheme with a group scheme action, viz. Coker $(a)$. Hence, it becomes necessary to consider quasi-projective schemes and projective spaces endowed with actions of the group schemes in sight. This addresses the part of the problem concerning the extension of the Tannakian methods (see Section 4).

In the geometric theory employed to interpret the group-theoretical directions, we turn to the study of projective stratified schemes and to the construction of a right adjoint to the obvious pullback functor

$$
f^{*}:\{\text { stratified schemes over } S\} \longrightarrow\{\text { stratified schemes over } X\} \text {. }
$$

(Strictly speaking, we content ourselves with the construction of a certain "universal object" instead of an adjoint; see Definition 15.1.) As we are mainly interested in stratified schemes, and not just stratified modules, it seemed pointless to study morphisms between topoi; we have opted to interpret the imagined adjoint as a manifestation of the Weil restriction [BLR90, 7.6, p. 191ff]. A blunt definition of the latter is simple enough to grasp: if $Z \rightarrow X$ is a scheme, its Weil restriction to $S$, denoted $f_{*} Z$, is the scheme of $S$-families of sections $\sigma: X \rightarrow Z$. Following the necessary adjustments, we arrive at the scheme of Section 10.

In order to treat stratified schemes, we thought important to bring in the concept of connections as imagined by Ehresmann [Ehr50], [CLN85, Chapter 5]. Ehresmann's connections are connections on fibre bundles which do not necessarily have an affine typical fibre and are best treated via the theory of foliations. Foliations then steer us to certain equivalence relations which are trivial in the topological sense and account only for nilpotent elements in the sheaves of rings: the infinitesimal equivalence relations; see Section 7. To get a good hold of these, the reader is advised to read Example 7.5 before anything. One advantage offered by working with equivalence relations is the possibility of making algebraic-geometric constructions as if they were banally set-theoretical. Another is the clear geometric way they give us the stratification on the scheme mentioned in the previous paragraph. Indeed, that scheme parametrizes certain families of sections, and we can imagine two among these to be equivalent if their images in the total space have equivalent points. See Definitions 11.1 and 11.3.

Here is an outline of the text.

Section 3 presents a proof of part (i) of Theorem 1.1. It is a straightforward exercise.

In Section 4 we introduce two criteria based on actions on projective spaces for a short sequence of affine group schemes to be exact. As said before, this is parallel to the criterion presented in [SGA1, V, $\S 6$ ], and the appearance of projective spaces is suggested by the fact that homogeneous spaces are quasi-projective. 


\section{HOMOTOPY EXACT SEQUENCE}

Section 5 deals with stratified schemes. It insists on the point of view that these stratified schemes are simply schemes endowed with an action of a "formal groupoid"; see $\S 5.2$. This idea seems to go back to Berthelot and Illusie; see [Ber74, Chapter II].

Section 6 takes up the idea of interpreting stratifications as actions of a formal groupoid of Section 5 and applies it to obtain instances where a stratified proper scheme "comes from the fundamental group scheme"; see Proposition 6.4. There the reader will also find a result which lets us conclude that stratified proper schemes are locally trivial for the faithfully flat of finite presentation (fppf) topology; see Lemma 6.2. This resembles the fact that stratified vector bundles are always locally free [BO78, Proposition 2.16].

The text then takes a different stand starting from Section 7, where we introduce infinitesimal equivalence relations (i.e.r.'s). This is one possible analogue of foliations [CLN85] in the algebraicgeometric setting. Such a path follows what seems to be Ehresmann's departure point for studying connections [Ehr50], although this view seems to have lost appeal in our particular branch due to the simplicity of the standard purely "abelian" definition $\nabla: E \rightarrow E \otimes \Omega^{1}$. Usually, to link the theory of foliations to algebraic geometry [Eke87, 3.1], [Miy87], one reinterprets the foliation as a sheaf of differential operators, but our choice insists in promoting the idea of orbits of vector fields.

Infinitesimal equivalence relations are simply equivalence relations which are trivial whenever points take values in reduced schemes. In Section 7 we only state definitions and prove basic properties of these. The introduction of the linearization (Definition 7.6) of an i.e.r. is particularly useful in the rest of the work, while the notion of total complementarity (Definition 7.10) shows how i.e.r.'s relate to stratifications. It should become clear to the reader that the notion of i.e.r. can be more useful than that of stratification.

In the next section, Section 8, we introduce a more specific and adapted kind of infinitesimal equivalence relation: the differentially smooth ones (Definition 8.1). These are more sophisticated analogues of foliations and mimic Grothendieck's definition of differential smoothness [EGA, IV 16.10]. In this important section, we observe that differentially smooth i.e.r.'s are locally given by formal actions of formal affine spaces and describe these precisely on the level of completions; see $\S \S 8.2,8.3$. This is parallel to the local interpretation of foliations as actions of affine spaces; see the appendix on the Frobenius theorem in [CLN85] and the proof of [ESB05, Theorem 2.7, p. 117].

Section 9 is devoted to the study of two fundamental properties of the theory: invariance and tangency. Continuing with the formal analysis made in $\S \S 8.2,8.3$, we study the shape of the complete local rings of an invariant subscheme of maximal codimension (a leaf); see Corollary 9.9. This study is in preparation for the analysis of the scheme of tangent sections.

In Section 10 we introduce the main character in the proof of Theorem 1.1: the relative scheme of tangent sections, or simply scheme of tangent sections, $H_{f}(Z)$. See Definition 10.4 and the lines following it. In the above notation, this scheme is the natural candidate for a "push-forward" of a stratified scheme $Z \rightarrow X$ along $f: X \rightarrow S$ :

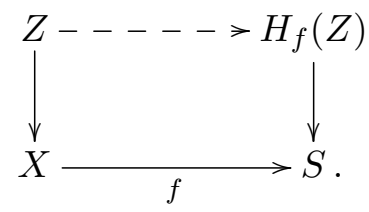

Its construction follows Grothendieck's analysis of the Weil restriction by means of Hilbert schemes [TDTE, IV, $\S 4 \mathrm{c}$, p. 19ff]. We note nonetheless that the construction of the scheme 


\section{J. P. DOS SANTOS}

of tangent sections works in a slightly more general setting than that of a stratification.

Building on the formal description of invariant subschemes of maximal codimension ( $\S \S 8.2$, 8.3), we show that under natural assumptions the scheme of tangent sections $H_{f}(Z)$ is proper over $S$ (Theorem 10.5). This characteristic usually escapes the Weil restriction.

In Section 11 we are concerned with the stratified structure of the scheme of tangent sections $H$ of the previous paragraph. In accordance with our principle that i.e.r.'s are more suitable objects than stratifications, we look for an i.e.r. on $H$. In doing so, we are led to present an equivalence relation among closed subschemes of a scheme endowed with an i.e.r. (Definition 11.3). This equivalence relation is the evident one: two closed subschemes are equivalent if their "orbits" intersect.

Section 12 concentrates in showing that the i.e.r. previously proposed turns the scheme of tangent sections $H_{f}(Z)$ into a universal object from $f^{*}$ to $Z$; see Definition 15.1. This property, though less popular, is the heart of "adjointness." Briefly, it implies that morphisms from an i.e.r. $(T, \Psi)$ over $S$, viz. a stratified $S$-scheme, to $H_{f}(Z)$ are in bijection with morphisms of i.e.r.'s $f^{*}(T, \Psi) \rightarrow Z$; see Corollary 12.3. From that, we deduce directly that the formation of $H$ commutes with base change; see Corollary 12.4.

Section 13 concentrates on showing that, under certain conditions, the infinitesimal equivalence relation defined on $H_{f}(Z)$ (see Section 11) comes from an $S$-stratification. This is condensed in Corollary 13.10 and stated in terms of "total complementarity" of infinitesimal equivalence relations (Definition 7.10). The basis of the argument is Proposition 13.4, which uses Hensel's lemma.

In Section 14 we study the scheme $H_{f}(Z)$ in the case where $S$ is a point. The obvious goal, in light of the "base change property" written in Corollary 12.4, is to gain knowledge concerning the fibres of the scheme of tangent sections over a positive-dimensional base. The main result is Proposition 14.5. To arrive at its statement, one important assumption on the stratified scheme $Z$ is made: it "comes from the fundamental group scheme"; see the introduction of $\S 14.2$ and the lines following Proposition 14.5. It should be noted that by imagining everything in terms of Ehresmann's theorem [CLN85, Chapter 5, Theorem 3, p. 99],

$$
\left\{\begin{array}{c}
\text { left actions of } \\
\text { the fundamental group }
\end{array}\right\} \cong\left\{\begin{array}{c}
\text { fibre bundles with } \\
\text { Ehresmann connections }
\end{array}\right\},
$$

the statement of Proposition 14.5 is no surprise.

In Section 15 we put the developed material together to furnish a proof of Theorem 1.1(ii). As mentioned earlier, the idea is to employ that the (relative) scheme of tangent sections $H_{f}(Z)$ of a certain stratified scheme $Z \rightarrow X$ (Section 10) is a "right adjoint" to- or better, a universal object from- $f^{*}$ of (1.1). (Such a property was made explicit in Section 12.) Through general nonsense (Lemma 15.2) we are then able to compare the fibre of $H_{f}(Z)$ above $s_{0} \in S(k)$ with the scheme of fixed points $\mathbb{P}(V)^{\operatorname{Ker}(b)}$; here $V$ is some representation of $\Pi(X)$. Such a manoeuvre is possible because $\mathbb{P}(V)^{\operatorname{Ker}(b)}$ is universal from

$$
\operatorname{Res}(b):\left\{\text { schemes with } \Pi\left(S, s_{0}\right) \text { action }\right\} \longrightarrow\left\{\text { schemes with } \Pi\left(X, x_{0}\right) \text { action }\right\}
$$

to $\mathbb{P}(V)$. This comparison, together with the depiction of $\left.H_{f}(Z)\right|_{s_{0}}$ from Section 14, allows us to verify the conditions for the equality $\operatorname{Ker}(b)=\operatorname{Im}(a)$ of Section 4 .

The article closes with Section 16, which tackles the question of checking the affinity of certain automorphism group schemes of projective schemes. It is written to permit the application of Proposition 6.4, which elucidates when a stratified scheme "comes from the fundamental group 


\section{HOMOTOPY EXACT SEQUENCE}

scheme".

We end this introduction with a brief review of recent literature on the "exact sequence". In [Hai13] the reader will find a proof of Theorem 1.1 for group schemes associated with $\Pi(-,-)$ and under the assumption $\operatorname{char}(k)>0$. In [Zha13], employing the complex analytic case, Zhang gives a proof of Theorem 1.1 under the assumption $\operatorname{char}(k)=0$. The case where $\Pi$ is replaced by Nori's fundamental group scheme was the subject of the incomplete work [EHV].

\section{Terminology}

(1) Given a morphism of ringed spaces $f:\left(X, \mathcal{O}_{X}\right) \rightarrow\left(Y, \mathcal{O}_{Y}\right)$, we denote the underlying morphism of topological spaces by $|f|$ and the accompanying morphism of rings $|f|^{-1} \mathcal{O}_{Y} \rightarrow \mathcal{O}_{X}$ by $f^{\#}$.

(2) For any scheme $X$, we let $X_{\text {red }}$ denote the reduced closed subscheme associated with $X$. See [EGA, I, Proposition 5.1.4, p. 128]. If $A$ is a ring, we let $A_{\text {red }}$ be the quotient of $A$ by its nilradical, for which we have $\operatorname{Spec}\left(A_{\text {red }}\right)=\operatorname{Spec}(A)_{\text {red }}$. Concerning this topic, we follow the conventions of [EGA, I].

(3) Let $B$ be a base scheme, let $S$ be a $B$-scheme, and let $G$ be a $B$-group scheme. Endow $S$ with the trivial $G$-action. A $G$-torsor over $S$ consists of an $S$-scheme $\psi: P \rightarrow S$ together with an action $\mu: P \times{ }_{B} G \rightarrow P$ on the right of $G$ such that (i) $\psi$ is faithfully flat and quasi-compact, and (ii) the obvious morphism $P \times G \rightarrow P \times{ }_{S} P$ is an isomorphism.

(4) Let $G$ and $H$ be affine group schemes over a field. A homomorphism $u: G \rightarrow H$ is called a quotient morphism if the induced arrow on rings $u^{\#}: \mathcal{O}(H) \rightarrow \mathcal{O}(G)$ is faithfully flat. Due to [Wat79, Theorem 14.1, p. 109], it suffices that $u^{\#}$ be injective.

(5) Given a group scheme $G$ over a field and a closed subgroup $H$, we let $G / H$ denote the fppf sheaf associated with the obvious functor [DG70, III.3.1.4, p. 324]. If $G$ is affine and algebraic, this sheaf is represented by an algebraic scheme [DG70, III.3.5.4, p. 341]. If $G$ is simply affine and $H$ is normal, then the obvious faithfully flat and quasi-compact (fpqc) sheaf $G / H$ is represented by an affine group scheme, as we learn from [SGA3, $\left.\mathrm{VI}_{\mathrm{B}}, 11.17\right]$.

\section{Surjectivity of $b: \Pi\left(X, x_{0}\right) \rightarrow \Pi\left(S, s_{0}\right)$}

We maintain the notation of Theorem 1.1. Let $\mathcal{D}_{\bullet}-\mathbf{m o d}$ denote the category of $\mathcal{O}_{\bullet}$-coherent $\mathcal{D} \bullet / k^{-m o d u l e s . ~ W e ~ r e m i n d ~ t h e ~ r e a d e r ~ t h a t, ~ d u e ~ t o ~ t h e ~ s m o o t h n e s s ~ o f ~} X$ and $S$, if $\mathcal{E} \in \mathcal{D}-\bmod$, then $\mathcal{E}$ is locally free over $\mathcal{O}$ [BO78, Proposition 2.16].

We wish to show that

(i) the functor

is fully faithful, and

$$
f^{*}: \mathcal{D}_{S^{-}} \bmod \longrightarrow \mathcal{D}_{X^{-}} \bmod
$$

(ii) the essential image of $\mathcal{D}_{S^{-}}$mod is stable under subobjects.

Together with [DM82, 2.21, p. 139], this shows part (i) of Theorem 1.1. We begin by noting that since $f_{*} \mathcal{O}_{X}=\mathcal{O}_{S}$, the functor $f^{*}$ from the category of locally free $\mathcal{O}_{S}$-modules to the category of locally free $\mathcal{O}_{X}$-modules is full and faithful. Thus, to show statement (i), we need to prove that if we are given $\mathcal{E}$ and $\mathcal{F}$ in $\mathcal{D}_{S}-\bmod$ and an $\mathcal{O}_{S}$-linear morphism $\varphi: \mathcal{E} \rightarrow \mathcal{F}$ such that $f^{*} \varphi$ is $\mathcal{D}_{X}$-linear, then $\varphi$ is $\mathcal{D}_{S}$-linear. This follows from direct local computations. Indeed, 


\section{J.P. DOS SAntos}

let $\left(s_{1}, \ldots, s_{m}\right): U \rightarrow \mathbb{A}_{k}^{m}$ be etale coordinates on an open subset of $S$. Let $V \subseteq X$ be an open subset mapping into $U$ and possessing functions $x_{1}, \ldots, x_{n}$ such that $(\boldsymbol{x}, \boldsymbol{s}): V \rightarrow \mathbb{A}_{k}^{m+n}$ is etale. Fix an arbitrary $\boldsymbol{p} \in \mathbb{N}^{m}$ and let $\partial^{[\boldsymbol{p}]} \in \mathcal{D}_{S}(U)$ be the differential operator satisfying

$$
\partial^{[p]}\left(s^{q}\right)=\left(\begin{array}{c}
\boldsymbol{q} \\
\boldsymbol{p}
\end{array}\right) \cdot \boldsymbol{s}^{\boldsymbol{q}-\boldsymbol{p}}
$$

see $\left[\right.$ EGA, $\mathrm{IV}_{4}$, Théorème 16.11.2, p. 54]. Let $\tilde{\partial}^{[\boldsymbol{p}]} \in \mathcal{D}_{X}(V)$ be the differential operator satisfying

$$
\tilde{\partial}^{[p]}\left(\boldsymbol{s}^{\boldsymbol{q}}\right)=\left(\begin{array}{c}
\boldsymbol{q} \\
\boldsymbol{p}
\end{array}\right) \cdot \boldsymbol{s}^{\boldsymbol{q}-\boldsymbol{p}} \quad \text { and } \quad \tilde{\partial}^{[\boldsymbol{p}]}\left(\boldsymbol{x}^{\boldsymbol{q}}\right)=0
$$

see [EGA, $\mathrm{IV}_{4}$, Théorème 16.11.2, p. 64]. It is not hard to show that for each $e \in \mathcal{E}(U)$, we have

$$
\tilde{\partial}^{[\boldsymbol{p}]} \cdot(1 \otimes e)=1 \otimes \partial^{[\boldsymbol{p}]} e .
$$

Using that $f$ is faithfully flat, we conclude that $\varphi$ is $\mathcal{D}_{S}$-linear.

The proof of statement (ii) also follows from a local calculation. Let $\mathcal{V}^{\prime} \in \mathcal{D}_{S^{-}}$mod and let $\mathcal{E}$ be a subobject in $\mathcal{D}_{X^{-}} \bmod$ of $f^{*} \mathcal{V}^{\prime}$. Let $s \in S$ be a closed point. Since $\left.\mathcal{V}^{\prime}\right|_{X_{s}}$ is a trivial $\mathcal{D}_{X_{s}}$-module, it follows that $\left.\mathcal{E}\right|_{X_{s}}$ is also trivial as a $\mathcal{D}_{X_{s}}$-module. In particular, $\left.\mathcal{E}\right|_{X_{s}}$ is trivial as an $\mathcal{O}_{X_{s}}$-module; this shows $\operatorname{dim} H^{0}\left(X_{s},\left.\mathcal{E}\right|_{X_{s}}\right)=\operatorname{rank} \mathcal{E}$. By [Mum70, II.5, Corollary 2, p. 50], $\mathcal{V}:=f_{*} \mathcal{E}$ is locally free and the restriction to $X_{s}$ of the canonical arrow of $\mathcal{O}_{X}$-modules

$$
\theta: f^{*} \mathcal{V} \longrightarrow \mathcal{E}
$$

is an isomorphism. As $f^{*} \mathcal{V}$ and $\mathcal{E}$ are locally free, it follows that $\theta$ is an isomorphism. Since $f^{*}$ is a fully faithful functor from the category of locally free $\mathcal{O}_{S}$-modules to the category of locally free $\mathcal{O}_{X}$-modules, there exists a unique $\mathcal{O}_{S}$-linear arrow $\sigma: \mathcal{V} \rightarrow \mathcal{V}^{\prime}$ such that $f^{*} \sigma$ is the composition $f^{*} \mathcal{V} \stackrel{\sim}{\rightarrow} \mathcal{G} f^{*} \mathcal{V}^{\prime}$.

By the faithful flatness of $f$, the arrow $\sigma$ is a monomorphism.

We now need to show that $\mathcal{V}$ possesses a $\mathcal{D}_{S}$-module structure which turns $\sigma$ into a morphism of $\mathcal{D}_{S}$-modules. Put differently, we need to show that $\operatorname{Im}(\sigma) \subseteq \mathcal{V}$ is invariant under $\mathcal{D}_{S}$. Once this is verified, the proof of statement (ii) is finished.

We will reduce the work to a local calculation using etale coordinates. Let $\left(s_{1}, \ldots, s_{m}\right): U \rightarrow$ $\mathbb{A}_{k}^{m}$ be etale coordinates on an open affine subset of $S$. Let $V \subseteq X$ be an open affine subset mapping into $U$ and possessing functions $x_{1}, \ldots, x_{n}$ such that $(\boldsymbol{x}, \boldsymbol{s}): V \rightarrow \mathbb{A}_{k}^{m+n}$ is etale. As in [EGA, $\mathrm{IV}_{4}$, Théorème 16.11.2, p. 54], for each multi-index $\boldsymbol{p} \in \mathbb{N}^{m}$, we write $\partial^{[\boldsymbol{p}]}$ for the differential operators on $U$ obtained from the functions $\boldsymbol{s}$, and for each multi-index $(\boldsymbol{p}, \boldsymbol{q}) \in$ $\mathbb{N}^{m} \times \mathbb{N}^{n}$, we write $\tilde{\partial}[\boldsymbol{p}, \boldsymbol{q}]$ for the differential operators on $V$ obtained from the functions $\boldsymbol{s}$ and $\boldsymbol{x}$. Moreover, we assume that $\mathcal{V}$ is free on the basis $v_{1}, \ldots, v_{h}$, while $\mathcal{V}^{\prime}$ is free on the basis $\sigma\left(v_{1}\right), \ldots, \sigma\left(v_{h}\right), v_{1}^{\prime}, \ldots, v_{c}^{\prime}$; this assumption poses no serious impediment since $f$ is faithfully flat and $\operatorname{Coker}\left(f^{*} \sigma\right)$ is locally free due to its $\mathcal{D}_{X}$-module structure. Then

$$
1 \otimes \partial^{[\boldsymbol{p}]} \sigma\left(v_{i}\right)=\tilde{\partial}^{[\boldsymbol{p}, \mathbf{0}]} \cdot\left(1 \otimes \sigma\left(v_{i}\right)\right)=\sum_{j=1}^{h} \xi_{i j} \cdot\left(1 \otimes \sigma\left(v_{j}\right)\right),
$$

where $\xi_{i j} \in \mathcal{O}_{X}(V)$. On the other hand, there exist functions $\alpha_{i j}, \beta_{i \ell} \in \mathcal{O}_{S}(U)$ such that $\partial^{[p]} \sigma\left(v_{i}\right)=\sum \alpha_{i j} \cdot \sigma\left(v_{j}\right)+\sum \beta_{i \ell} \cdot v_{\ell}^{\prime}$, so that $\xi_{i j}=f^{\#} \alpha_{i j}$ and $f^{\#} \beta_{i \ell}=0$. By the flatness of $f^{\#}$, we conclude that $\beta_{i \ell, P}=0$ for each $P \in f(V)$. This shows that $\operatorname{Im}(\sigma)$ is invariant under $\mathcal{D}_{S}$. 


\section{Homotopy EXACT SEQUENCE}

\section{Criterion for exactness using projective representations}

We fix an algebraically closed field $k$. All morphisms and objects are over $k$. Let

$$
K \stackrel{a}{\longrightarrow} \Pi \stackrel{b}{\longrightarrow} H
$$

be homomorphisms of affine group schemes such that $b \circ a$ is the trivial homomorphism and $b$ is a quotient morphism.

4.1. We define a property concerning the morphisms $a$ and $b$.

Property 4.1. For all affine algebraic quotients $q: \Pi \rightarrow G$ and all closed subgroup schemes $I \leqslant G$,

$$
(G / I)^{K} \neq \emptyset \quad \Longrightarrow \quad \operatorname{Ker}(b) \text { acts trivially on } G / I .
$$

Remark 4.1. Let $N \preccurlyeq \Pi$ be a closed subgroup. Let $q: \Pi \rightarrow G$ be a quotient morphism of affine group schemes. Then $N$ acts trivially on $G / I$ if and only if $q: N \rightarrow G$ factors through $I$. The proof of this fact is an exercise using [DG70, III.3.1.5, p. 324] and the fact that $\mathcal{O}(\Pi)$ is a direct limit of faithfully flat $\mathcal{O}(G)$-algebras [Wat79, 3.3 and 14.1].

Lemma 4.2. Property 4.1 holds if and only if $\operatorname{Ker}(b) \subseteq \operatorname{Im}(a)$.

Proof. $(\Rightarrow)$ Let $q: \Pi \rightarrow G$ be an affine and algebraic quotient. Let $I \leqslant G$ be the image of $q \circ a: K \rightarrow G$. There exists a $K$-invariant element in $G / I$ determined by the image of the identity

$$
\operatorname{Spec}(k) \stackrel{e_{G}}{\rightarrow} G \longrightarrow G / I \text {. }
$$

So $\operatorname{Ker}(b)$ acts trivially on $G / I$. Hence, $q: \operatorname{Ker}(b) \rightarrow G$ factors though $I$. The implication then follows, not without a small effort, from the fact that the Hopf algebra $\mathcal{O}(\Pi)$ is a direct limit of Hopf algebras which are finitely generated over $k$ [Wat79, 3.3].

$(\Leftarrow)$ Assume $\operatorname{Ker}(b)=\operatorname{Im}(a)$. Consequently, $\operatorname{Im}(a) \preccurlyeq \Pi$. Let $q: \Pi \rightarrow G$ be a faithfully flat homomorphism and let $I \leqslant G$ be a closed subgroup such that $(G / I)^{K} \neq \emptyset$. We claim that $q: \operatorname{Ker}(b) \rightarrow G$ factors through $I \rightarrow G$. Let $\mathfrak{p}: G \rightarrow G / I$ be the canonic morphism. Since $G / I$ is algebraic over $k$, and $\mathfrak{p}(k): G(k) \rightarrow(G / I)(k)$ is surjective, there exists a $g \in G(k)$ such that $\mathfrak{p}(g) \in(G / I)(k)$ is $K$-invariant. It also possible to assume $g=q(\gamma)$ for some $\gamma \in \Pi(k)$. Now for any $k$-algebra $R$ and any $y \in K(R)$, we have

$$
\mathfrak{p}\left(g^{-1} \cdot q a(y) \cdot g\right)=g^{-1} \cdot \mathfrak{p}(q a(y) \cdot g)=g^{-1} \cdot \mathfrak{p}(g)=\mathfrak{p}(e) .
$$

As the inverse image of $\mathfrak{p}(e)$ under $\mathfrak{p}(R): G(R) \rightarrow(G / I)(R)$ is $I(R)$ [DG70, III.3.1.5, p. 324], it follows that $g^{-1} \cdot q a \cdot g: K \rightarrow G$ factors though $I$. Using $\operatorname{Im}(a) \preccurlyeq \Pi$ and $g=q(\gamma)$, it is easy to conclude that $q a: K \rightarrow G$ factors through $I$.

4.2. Let us define another property. The arrows $a: K \rightarrow \Pi$ and $b: \Pi \rightarrow H$ are as before.

Property 4.2. For any finite-dimensional representation $V$ of $\Pi$,

$$
\mathbb{P}(V)^{\operatorname{Im}(a)}(k) \subseteq \mathbb{P}(V)^{\operatorname{Ker}(b)}(k) .
$$

Here we use the notation and definitions of [DG70, II.1.3.4, p. 163] (or [Jan87, I, 2.6, p. 27ff]) concerning schemes of fixed points. It is opportune to observe that Property 4.2 can be translated as forcing the equality of topological spaces

$$
\left|\mathbb{P}(V)^{\operatorname{Ker}(b)}\right|=\left|\mathbb{P}(V)^{\operatorname{Im}(a)}\right|,
$$




\section{J. P. DOS SANTOS}

as these fixed-point functors are all represented by closed subschemes of $\mathbb{P}(V)$ [DG70, II.1.3.6(d), p. 165].

\section{Lemma 4.3. Property 4.2 is equivalent to Property 4.1.}

Proof. $(4.2 \Rightarrow 4.1)$ Let $q: \Pi \rightarrow G$ be a faithfully flat homomorphism to an algebraic group scheme, and let $I \leqslant G$ be a closed subgroup. We assume $(G / I)^{K} \neq \emptyset$. By Chevalley's theorem, there exist a representation $V$ of $G$ and an equivariant immersion $G / I \rightarrow \mathbb{P}(V)$, as indicated in the proof of [DG70, III.3, Theorem 5.4]. Denote by $\mathfrak{p}$ the canonical morphism of schemes $G \rightarrow G / I$, and let $g \in G(k)$ be such that $\mathfrak{p}(g)$ is $K$-invariant. By hypothesis, $\mathfrak{p}(g)$ is also $\operatorname{Ker}(b)$-invariant. This means that for each $k$-algebra $R$ and each $y \in \operatorname{Ker}(b)(R)$, we have

$$
\begin{aligned}
q(y) \cdot \mathfrak{p}(g)=\mathfrak{p}(g) & \Rightarrow\left(g^{-1} \cdot q(y) \cdot g\right) \cdot \mathfrak{p}(e)=\mathfrak{p}(e) \\
& \Rightarrow g^{-1} \cdot q \cdot g: \operatorname{Ker}(b) \rightarrow G \text { factors though } I,
\end{aligned}
$$

due to [DG70, III.3.1.5, p. 324]. As $q: \Pi(k) \rightarrow G(k)$ is surjective and $\operatorname{Ker}(b)$ is normal, it follows that $q: \operatorname{Ker}(b) \rightarrow G$ factors though $I$. By Remark 4.1, the kernel $\operatorname{Ker}(b)$ acts trivially on $G / I$.

$(4.2 \Leftarrow 4.1)$ By virtue of Lemma 4.2 this is clear.

\section{Stratified schemes}

\subsection{Groupoids and actions of groupoids}

Let $\mathcal{C}$ be a category; from now on, we assume that all displayed fibered products exist in $\mathcal{C}$. Let

$$
G \underset{p_{1}}{\stackrel{p_{0}}{\longrightarrow}} S, \quad \delta: G \underset{p_{0}, S, p_{1}}{\times} G \longrightarrow G, \quad \sigma: G \longrightarrow G, \quad \varepsilon: S \longrightarrow G
$$

be a groupoid in $\mathcal{C}[\mathrm{MM} 03,5.1]$. The source of $\delta$, the object of "composable" arrows, will be denoted by $G(2)$. This object is also the source of other two arrows:

$$
\begin{aligned}
& \chi_{0}: G(2) \longrightarrow G, \text { the first projection, } \\
& \chi_{1}: G(2) \longrightarrow G, \text { the second projection. }
\end{aligned}
$$

These arrows satisfy

$$
\begin{gathered}
p_{0} \circ \delta=p_{0} \circ \chi_{1}, \quad p_{1} \circ \delta=p_{1} \circ \chi_{0}, \quad p_{1} \circ \chi_{1}=p_{0} \circ \chi_{0}, \\
p_{0} \varepsilon=p_{1} \varepsilon=\operatorname{Id}_{S}, \quad p_{0} \sigma=p_{1}, \quad p_{1} \sigma=p_{0} .
\end{gathered}
$$

Remark 5.1. There is some discrepancy in the literature concerning the definition of the object of "composable" arrows. We have followed the one which preserves graphical simplicity, saying that $\delta\left(g, g^{\prime}\right)$ should be thought of as $g \circ g^{\prime}$. This is what [MM03] adopts as definition. On the other hand, following Grothendieck [TDTE, III], it is common to work with the switched object of "composable" arrows, that is, $G \times_{p_{1}, S, p_{0}} G$. Berthelot [Ber74] also follows this convention. Further ahead we will find convenient to follow it.

We now want to study left actions of $G$. Given an object $\pi: E \rightarrow S$ over $S$, let

$$
\alpha: G \underset{p_{0}, S, \pi}{\times} E \longrightarrow E
$$

be an arrow satisfying

A1. $\pi \alpha(g, y)=p_{1}(g)$ for all $g \in G$ and $y \in E$.

A2. $\alpha(\varepsilon(\pi(y)), y)=y$ for all $y \in E$. 


\section{HOMOTOPY EXACT SEQUENCE}

Condition A1 amounts to there being an arrow

$$
\theta:=\left(\operatorname{pr}_{G}, \alpha\right): G \underset{p_{0}, S, \pi}{\times} E \longrightarrow G \underset{p_{1}, S, \pi}{\times} E
$$

of $G$-objects. Due to (5.2), pulling back $\theta$ by $\varepsilon: S \rightarrow G$ defines an arrow $E \rightarrow E$ and condition A2 just means that this is $\operatorname{id}_{E}$. Pulling back $\theta$ by $\chi_{1}$, we derive an arrow of $G(2)$-objects

$$
\chi_{1}^{*}(\theta): G(2) \underset{p_{0} \chi_{1}, S, \pi}{\times} E \longrightarrow G(2) \underset{p_{1} \chi_{1}, S, \pi}{\times} E, \quad(g, h, y) \longmapsto(g, h, \alpha(h, y)) .
$$

Pulling back $\theta$ by $\chi_{0}$, we obtain

$$
\chi_{0}^{*}(\theta): G(2) \underset{p_{0} \chi_{0}, S, \pi}{\times} E \longrightarrow G(2) \underset{p_{1} \chi_{0}, S, \pi}{\times} E, \quad(g, h, y) \longmapsto(g, h, \alpha(g, y)) .
$$

Pulling back $\theta$ by $\delta$, we obtain

$$
\delta^{*}(\theta): G(2) \underset{p_{0} \delta, S, \pi}{\times} E \longrightarrow G(2) \underset{p_{1} \delta, S, \pi}{\times} E, \quad(g, h, y) \longmapsto(g, h, \alpha(\delta(g, h), y)) .
$$

By means of (5.1), we see that $\delta^{*}(\theta)$ and $\chi_{0}^{*}(\theta) \circ \chi_{1}^{*}(\theta)$ have the same source and target in $\mathcal{C}$. The definition presented in [MM03, 5.3, p. 125] can now be given the following translation.

Definition 5.2. We say that $\alpha$ is a left action of $G$ on $\pi: E \rightarrow S$ if, together with conditions A1 and $\mathrm{A} 2$, we have

A3. $\delta^{*}(\theta)=\chi_{0}^{*}(\theta) \circ \chi_{1}^{*}(\theta)$.

Lemma 5.3. Let $\alpha$ be a left action of $G$ on $\pi: E \rightarrow S$. Then the arrow $\theta$ is an isomorphism.

Proof. We assume that the category $\mathcal{C}$ is a category of sets. Let $\theta(g, y)=\theta\left(g^{\prime}, y^{\prime}\right)$; by definition $g=g^{\prime}$ and $\alpha(g, y)=\alpha\left(g, y^{\prime}\right)$. As $\alpha(\sigma(g), \alpha(g, z))=\alpha(\varepsilon(\pi(z)), z)=z$, we obtain $y=y^{\prime}$. Thus $\theta$ is injective. We now prove surjectivity. Let $(g, y) \in G \times E$ be such that $p_{1}(g)=\pi(y)$. Since $p_{0}(\sigma(g))=p_{1}(g)$, we can consider the element $y^{\prime}:=\alpha(\sigma(g), y)$. Now, $\pi\left(y^{\prime}\right)=p_{1}(\sigma(g))=p_{0}(g)$. Then $\alpha\left(g, y^{\prime}\right)=\alpha(g, \alpha(\sigma(g), y))=y$.

In view of this easy lemma, Definition 5.4 below agrees with Definition 5.2.

Definition 5.4. An action of $G$ on $\pi: E \rightarrow S$ is an isomorphism of $G$-objects

$$
\theta: G \times_{p_{0}, S, \pi} E \stackrel{\simeq}{\longrightarrow} G \times_{p_{1}, S, \pi} E
$$

which satisfies $\varepsilon^{*}(\theta)=\mathrm{id}_{E}$ and axiom A3.

In more functional fashion, an action $\theta$ of the groupoid $G$ on $\pi: E \rightarrow S$ determines for each $g \in G(U)=\operatorname{Hom}(U, G)$ a $U$-isomorphism

$$
\theta_{g}: p_{0}(g)^{-1} E \stackrel{\sim}{\longrightarrow} p_{1}(g)^{-1} E,
$$

and these satisfy

$$
\begin{array}{ll}
\theta_{g^{\prime} g}=\theta_{g^{\prime}} \circ \theta_{g} & \text { if } p_{0}\left(g^{\prime}\right)=p_{1}(g), \text { and } \\
\theta_{\varepsilon(s)}=\operatorname{id}_{s^{-1} E} & \text { for each } s \in S(U) .
\end{array}
$$

\subsection{The formal diagonal groupoid and stratifications}

Let us fix a noetherian separated scheme $B$, and let $S$ be a $B$-scheme that is locally of finite type and separated. Let Sch denote the category of locally noetherian $B$-schemes and FSch the category of locally noetherian formal $B$-schemes. We regard $\mathbf{S c h}$ as a full subcategory of 


\section{J. P. DOS SANTOS}

FSch [EGA, I, 10.4.8, p. 186] and the latter as a full subcategory of Set $^{\text {Sch }}{ }^{\text {op }}$ following [ML98, X, $\S 6$, Proposition 2, p. 246] and [EGA, I, 10.6, p. 188ff]. Even though general fibre products do not exist in FSch, those considered in this text do exist. (It is profitable to recall that if $\mathfrak{X}$ is an adic formal scheme with ideal of definition $\mathcal{J}$ such that $(|\mathfrak{X}|, \mathcal{O} / \mathcal{J})$ is a locally noetherian scheme and the ideal $\mathcal{J} / \mathcal{J}^{2}$ is of finite type as a $\mathcal{O} / \mathcal{J}$-module, then $\mathfrak{X}$ is locally noetherian [EGA, $\mathrm{I}_{0}$, Corollaire 7.2.6, p. 64].) References to $B$ will be omitted as much as possible; for example, $\times$ will stand for $\times_{B}$.

We wish to harmonize Berthelot's terminology in [Ber74, Chapter II] with ours, so our groupoids will have the switched object of "composable" arrows for the moment. See Remark 5.1.

Let $S^{\prime}:=S \times S$ and $S^{\prime \prime}:=S \times S \times S$. Denote the obvious projections $S^{\prime} \rightarrow S$ by $\dot{p}_{0}$ and $\dot{p}_{1}$. Define the following morphisms

$$
\begin{aligned}
\dot{q}_{0}: S^{\prime \prime} \longrightarrow S^{\prime}, & \left(s_{1}, s_{2}, s_{3}\right) \longmapsto\left(s_{1}, s_{2}\right), \\
\dot{q}_{1}: S^{\prime \prime} \longrightarrow S^{\prime}, & \left(s_{1}, s_{2}, s_{3}\right) \longmapsto\left(s_{2}, s_{3}\right), \\
\dot{\delta}: S^{\prime \prime} \longrightarrow S^{\prime}, & \left(s_{1}, s_{2}, s_{3}\right) \longmapsto\left(s_{1}, s_{3}\right) .
\end{aligned}
$$

Note that by means of the identification

$$
S^{\prime \prime}=S^{\prime} \times_{\dot{p}_{1}, S, \dot{p}_{0}} S^{\prime}, \quad\left(s_{1}, s_{2}, s_{3}\right) \longmapsto\left[\left(s_{1}, s_{2}\right) ;\left(s_{2}, s_{3}\right)\right],
$$

the morphisms $\dot{q}_{0}$ and $\dot{q}_{1}$ correspond to the first and second projections, respectively. Finally, let $\dot{\varepsilon}: S \rightarrow S^{\prime}$ denote the diagonal morphism and $\dot{s}: S^{\prime} \rightarrow S^{\prime}$ the "switch" morphism. We then obtain an obvious groupoid, sometimes called the kernel groupoid [MM03, 5.1(2), p. 113]:

$$
S^{\prime} \underset{\dot{p}_{1}}{\stackrel{\dot{p}_{0}}{\longrightarrow}} S, \quad \dot{\delta}: S^{\prime \prime} \longrightarrow S^{\prime}, \quad \dot{s}: S^{\prime} \longrightarrow S^{\prime}, \quad \dot{\varepsilon}: S \longrightarrow S^{\prime} .
$$

We now pass to formal schemes. Let $\left|\Delta^{\prime}\right|$, respectively $\left|\Delta^{\prime \prime}\right|$, denote the closed subspace of $S^{\prime}$, respectively $S^{\prime \prime}$, corresponding to the diagonal. Let

$$
\widehat{P}_{S} \text { and } \widehat{P}_{S}(2)
$$

denote the completion of $S^{\prime}$ along $\left|\Delta^{\prime}\right|$ and the completion of $S^{\prime \prime}$ along $\left|\Delta^{\prime \prime}\right|$, respectively; see [EGA, I, 10.8, p. 194ff].

Since $\dot{\varepsilon}(S) \subseteq\left|\Delta^{\prime}\right|, \dot{s}\left(\left|\Delta^{\prime}\right|\right) \subseteq\left|\Delta^{\prime}\right|, \dot{q}_{i}\left(\left|\Delta^{\prime \prime}\right|\right) \subseteq\left|\Delta^{\prime}\right|$, and $\dot{\delta}\left(\left|\Delta^{\prime}\right|\right) \subseteq\left|\Delta^{\prime \prime}\right|$, we deduce morphisms

$$
p_{i}: \widehat{P}_{S} \longrightarrow S, \quad \varepsilon: S \longrightarrow \widehat{P}_{S}, \quad \delta: \widehat{P}_{S}(2) \longrightarrow \widehat{P}_{S}, \quad \text { etc. }
$$

Note that (5.4) and [EGA, I, Proposition 10.9.7, p. 200] allow us to identify

$$
\widehat{P}_{S}(2)=\widehat{P}_{S} \times_{p_{1}, S, p_{0}} \widehat{P}_{S},
$$

$q_{0}$ with the first projection, and $q_{1}$ with the second. (In particular, the fibre product in question exists in FSch.) It is interesting to note that the restriction $\delta: P_{S}^{\nu} \times_{S} P_{S}^{\nu} \rightarrow \widehat{P}_{S}$ does not factor through $P_{S}^{\nu}$, a fact which hides the property that the composition of differential operators of order $\nu$ will not usually be of order $\nu$; see [EGA, $\mathrm{IV}_{4}$, Lemme 16.8.9.1, p. 43]. By "functoriality of completions" [EGA, I, 10.9.3, p. 199], the arrows in (5.5) together with the identification $\widehat{P}_{S}(2)=\widehat{P}_{S} \times_{p_{1}, p_{0}} \widehat{P}_{S}$ give us a groupoid

$$
\widehat{P}_{S} \underset{p_{1}}{\stackrel{p_{0}}{\longrightarrow}} S, \quad \delta: \widehat{P}_{S} \times_{p_{1}, p_{0}} \widehat{P}_{S} \longrightarrow \widehat{P}_{S}, \quad \varepsilon: S \longrightarrow \widehat{P}_{S}, \quad s: \widehat{P}_{S} \longrightarrow \widehat{P}_{S}
$$

in FSch, which we call the formal diagonal groupoid. We now want to make explicit the fact that actions of the formal diagonal groupoid are simply the stratifications of [Ber74]. 


\section{Homotopy EXACT SEQUENCE}

Let $P_{S}^{\nu}$ be the $\nu$ th infinitesimal neighbourhood of the diagonal in $S \times S$; see [EGA, $\mathrm{IV}_{4}$, Définitions 16.1 .2 , p. 5 and 16.3 .1$, p. 14]. Note that

$$
\widehat{P}_{S}=\underset{\nu}{\lim } P_{S}^{\nu},
$$

as explains [EGA, I, 10.6]. The schemes $P_{S}^{\nu}$ come with two evident morphisms to $S$, denoted $p_{0}^{\nu}$ and $p_{1}^{\nu}$, and one from $S$, denoted $\varepsilon^{\nu}: S \rightarrow P_{S}^{\nu}$. Analogously, let $P_{S}^{\nu}(2)$ denote the $\nu$ th infinitesimal neighbourhood of the diagonal in $S^{\prime \prime}=S \times S \times S$. Write $q_{0}^{\nu}, q_{1}^{\nu}$ and $\delta^{\nu}$ for the morphisms from $P_{S}^{\nu}(2)$ to $P_{S}^{\nu}$ induced by $\dot{q}_{0}, \dot{q}_{1}$, and $\dot{\delta}$ as above, respectively. Berthelot [Ber74, II.1.3, p. 96] defines a stratification of $\pi$ to be a family of compatible isomorphisms

$$
\theta_{\nu}: p_{0}^{\nu *} E \stackrel{\sim}{\longrightarrow} p_{1}^{\nu *} E
$$

of $P_{S}^{\nu}$-schemes which satisfies

$$
\varepsilon^{\nu *}\left(\theta_{\nu}\right)=\operatorname{id}_{E}, \quad \delta^{\nu *}\left(\theta_{\nu}\right)=q_{1}^{\nu *}\left(\theta_{\nu}\right) \circ q_{0}^{\nu *}\left(\theta_{\nu}\right), \quad \forall \nu \in \mathbb{N} .
$$

(Note that in [Ber74, II.1.1.3, p. 84], Berthelot prefers to work with the algebras instead of the schemes, so, in the preceding discussion, our $q_{i}^{\nu}$ is his Spec $q_{i}^{\nu}$.) Due to [EGA, I, 10.6.7, p. 191 and 10.7.4, p. 193], such families are in bijection with isomorphism

$$
\theta: E \times_{S, p_{0}} \widehat{P}_{S} \stackrel{\sim}{\longrightarrow} \widehat{P}_{S} \times_{p_{1}, S} E
$$

in $\mathbf{F S c h} / \widehat{P}_{S}$ satisfying $\varepsilon^{*}(\theta)=\mathrm{id}_{E}$ and

$$
\delta^{*}(\theta)=q_{1}^{*}(\theta) \circ q_{0}^{*}(\theta) .
$$

If we now reinstate our conventions of $\S 5.1$ and switch the factors in the objects of "composable" arrows, that is, define $\widehat{P}_{S}(2)$ as $\widehat{P}_{S} \times_{p_{0}, p_{1}} \widehat{P}_{S}$, then $q_{0}$ corresponds to what was $\chi_{1}$ in $\S 5.1$ and $q_{1}$ to $\chi_{0}$; consequently, (5.6) is equivalent to condition A3 of $\S 5.1$. All these trivial observations only find their raison d'être in sustaining the following.

Lemma 5.5. Let $\pi: E \rightarrow S$ be a morphism of schemes. The data of a $B$-linear stratification of $\pi$ is a left action (see Definition 5.4) of the formal diagonal groupoid on $E$.

The category of $B$-linearly stratified schemes over $S$ will be denoted by $\mathbf{S t r S}(S / B)$, and when there is no risk of confusion, we shall drop the reference to the base $B$. In the same spirit of economy and simplification, we shall adopt the following convention of Berthelot.

Convention 5.6. Whenever considering a fibre product in which one of the factors is $P_{S}^{\nu}$ (or $\widehat{P}_{S}$ ) we will put the symbol $P_{S}^{\nu}$ on the left to mean that the second projection is being considered, and on the right if the first projection is being used to form the product. For example, $P_{S}^{\nu} \times{ }_{S} X$ means that we consider $P_{S}^{\nu}$ as a scheme over $S$ via $p_{1}^{\nu}$ in order to form the fibre product.

\section{A non-affine Tannakian correspondence}

We fix an algebraically closed field $k$. In this section, Sch, respectively FSch, denotes the category of locally noetherian $k$-schemes, respectively locally noetherian formal $k$-schemes.

Let $S$ be a smooth and connected $k$-scheme. We fix $s_{0} \in S(k)$. Let $\mathcal{D}_{S}$ denote the ring of $k$-linear differential operators on $S$; see [EGA, IV 4 , Définition 16.3.1, p. 14] or [BO78, §2].

Our goal in this section is to study the relation between the stratified $S$-schemes of Section 5 and $\mathcal{O}_{S}$-coherent $\mathcal{D}_{S}$-modules. 


\section{J. P. DOS SANTOS}

\section{1 $\mathcal{O}_{S}$-coherent $\mathcal{D}_{S}$-modules}

The category of $\mathcal{D}_{S}$-modules, $\mathcal{D}_{S}-$ Mod, is an abelian category endowed with a tensor product. Its full subcategory consisting of those modules which are coherent over $\mathcal{O}_{S}$ will be denoted $\mathcal{D}_{S}-\bmod$ from now on. The functor

$$
s_{0}^{*}: \mathcal{D}_{S}-\mathbf{m o d} \longrightarrow k-\bmod , \quad \mathcal{M} \longmapsto \mathcal{M} \otimes \boldsymbol{k}\left(s_{0}\right)
$$

endows $\mathcal{D}_{S}-\mathbf{m o d}$ with a structure of neutral Tannakian category [DM82, § 2], and the affine group scheme associated with this data is denoted by $\Pi\left(S, s_{0}\right)$; see [DM82, Theorem 2.11, p. 130] for the construction of $\Pi\left(S, s_{0}\right)$, [SR72, VI, 1.2.2] for one of its earliest appearances, and [dSan07] for more basic properties. It is common to call $\Pi\left(S, s_{0}\right)$ the stratified fundamental group scheme, and we will refer to it simply as the fundamental group scheme of $S$. To ease the notation, we will write $\Pi(S)$ instead of $\Pi\left(S, s_{0}\right)$.

Let $\psi: \mathcal{U}_{S} \rightarrow S$ be the universal $\Pi(S)$-torsor as constructed in [dSan07, 2.3.2], or in [Nor76, $\S 2]$. This is the object of the ind-category of $\mathcal{D}_{S}$-modules corresponding to the left-regular representation of $\Pi(S)$. Note that the morphism $\psi$ is affine, so that $\psi$ amounts to the data of the quasi-coherent $\mathcal{O}_{S}$-algebra $\psi_{*} \mathcal{O}_{\mathfrak{U}_{S}}$. This $\mathcal{O}_{S}$-algebra is in fact a particular kind of $\mathcal{D}_{S}$-module, which we now set to describe.

First, just as for affine group schemes,

$$
\mathcal{U}_{S}=\underbrace{\lim }_{\lambda} \mathcal{U}_{\lambda}
$$

where $\psi_{\lambda}: \mathcal{U}_{\lambda} \rightarrow S$ is a torsor under an algebraic affine group $\Pi(S)_{\lambda}$ and the arrows in the projective systems are all morphisms of torsors. Each $\mathcal{U}_{\lambda}$ is a stratified torsor over $S$, the meaning of which we recall. For each $\lambda$, there exists a $k$-linear stratification of $\psi_{\lambda}$,

$$
\left\{\alpha_{\lambda, \nu}: \mathfrak{U}_{\lambda} \times_{S} P_{S}^{\nu} \longrightarrow \mathcal{U}_{\lambda}: \nu \in \mathbb{N}\right\}
$$

such that the canonical isomorphism

$$
\mathcal{U}_{\lambda} \times \Pi(S) \stackrel{\sim}{\longrightarrow} \mathcal{U}_{\lambda} \times{ }_{S} \mathcal{U}_{\lambda}, \quad(u, \gamma) \longmapsto(u, u \cdot \gamma)
$$

sends the standard stratification on $\mathcal{U}_{\lambda} \times \Pi(S)$, that is, the stratification induced by the stratification on $\mathcal{U}_{\lambda}$, to the product stratification on $\mathcal{U}_{\lambda} \times_{S} \mathcal{U}_{\lambda}$. In addition, the transition morphisms in the projective family $\mathcal{U}_{\lambda}$ are morphisms of stratified schemes.

Of course, all these conditions can be translated into "abelian terms" upon passage to the category of quasi-coherent $\mathcal{O}_{S}$-algebras; this is explained in [dSan07, 2.3.1, 2.3.2]. Due to the fact that each $\Pi(S)_{\lambda}$-module is a direct limit of finite-dimensional $\Pi(S)_{\lambda}$-modules [Wat79, 3.3, p. 24], it follows that the $\mathcal{D}_{S}$-module $\mathcal{O}_{\mathcal{U}_{\lambda}}$ is a direct limit of $\mathcal{O}_{S}$-coherent $\mathcal{D}_{S}$-modules. A fortiori $\mathcal{O}_{\mathcal{U}_{S}}=\lim _{\mathcal{O}_{\mathcal{U}_{\lambda}}}$ is a direct limit of objects in $\mathcal{D}_{S^{-}} \mathbf{m o d}$. The role of the fundamental group scheme $\overrightarrow{\Pi(S)}$ and $\mathcal{U}_{S}$ is to control all $\mathcal{D}_{S}$-modules of this particular type.

\subsection{Proper stratified schemes}

Let $\operatorname{Str} \mathbf{P}(S)$ be the full subcategory of $\operatorname{StrS}(S)$ (see Section 5) consisting of those objects whose underlying $S$-scheme is proper. One simple way to construct objects in this category is by contraction with $\mathcal{U}_{S}$. Choose a proper $k$-scheme $F$ together with a representation $\rho: \Pi(S) \rightarrow$ $\operatorname{Aut}_{k}(F)$; assume that the quotient of $\mathcal{U}_{S} \times F$ by the diagonal action of $\Pi(S)$ on the right is a scheme

$$
\mathcal{U}_{S} \times^{\rho} F \longrightarrow S
$$


(A good source for learning the gymnastics around contracted products $\bullet \times^{\rho} \bullet$ is [Jan87, Part 1, Chapter 5].) The stratification of $\mathcal{U}_{S} \times F$ "on the first factor" then gives a stratified $S$-scheme $\mathcal{U}_{S} \times{ }^{\rho} F \rightarrow S$; this can be seen using the two following arguments: (i) There is an obvious stratification on the presheaf $\left(\mathcal{U}_{S} \times F\right) / \Pi(S): \mathbf{S c h}^{o} \rightarrow$ Set. (ii) Any stratification on a presheaf induces one on the associated sheaf, due to the fact that "taking the associated sheaf commutes with limits". (This last statement is explained in [SGA4]; see [SGA4, II, Proposition 4.3, p. 237 and I, Définition 2.5, p. 13]) Moreover, flat descent [EGA, $\mathrm{IV}_{2}$, Proposition 2.7.1, p. 29] ensures that $\mathcal{U}_{S} \times{ }^{\rho} F \rightarrow S$ is proper, so that $\mathcal{U}_{S} \times{ }^{\rho} F \in \mathbf{S t r P}(S)$.

Remark 6.1. Of course, restrictions are needed for the quotient $\left(\mathcal{U}_{S} \times F\right) / \Pi(S)$ to be a scheme. Two important instance in which this occurs are the case where $F$ is affine and that where there exist a finite-dimensional $\Pi(S)$-module $V$ and a $\Pi(S)$-equivariant immersion $F \rightarrow \mathbb{P}(V)$. The first class of examples follows directly from [TDTE, I, Théorème 2], while the second follows from [BLR90, §6.2, Theorem 7, p. 138].

Our goal is to introduce some elementary constructions which will allow us to identify some objects of $\operatorname{StrP}(S)$ arising from $\mathfrak{U}_{S}$. For this matter, let

$$
\mathcal{R}_{S}=\left\{\begin{array}{l}
\text { pairs }\left(F, \rho: \Pi(S) \rightarrow \mathbf{A u t}_{k}(F)\right) \text { consisting of a proper } k \text {-scheme } F \\
\text { and a left action } \rho \text { of } \Pi(S) \text { on } F \text { such that } \mathcal{U}_{S} \times^{\rho} F \text { is a scheme }
\end{array}\right\} .
$$

Together with the obvious arrows, $\mathcal{R}_{S}$ is a category and $(F, \rho) \mapsto \mathcal{U}_{S} \times^{\rho} F$ is a functor to $\operatorname{Str} \mathbf{P}(S)$. Objects of $\operatorname{StrP}(S)$ stemming from $\mathcal{R}_{S}$ are called stratified proper schemes coming from the fundamental group scheme.

\subsection{Local triviality of proper stratified schemes}

The following result is reminiscent of the fact that coherent modules with stratifications are locally free $[\mathrm{BO} 78,2.16]$.

Lemma 6.2. Let $\left(\pi: E \rightarrow S,\left\{\theta_{\nu}\right\}\right)$ be a stratified scheme and assume that $\pi$ is proper. Let $F$ denote the fibre of $\pi$ above $s_{0}$. Then, there exist an fppf covering $S^{\prime} \rightarrow S$ and an isomorphism

$$
S^{\prime} \times{ }_{S} E \stackrel{\simeq}{\longrightarrow} S^{\prime} \times F .
$$

In particular, $\pi$ is flat.

Proof. Let $\widehat{E}_{s_{0}}$ be the formal fibre of $\pi$ at $s_{0}$. Using the stratification, we can easily construct an isomorphism of formal schemes over $\operatorname{Spf} \widehat{\mathcal{O}}_{s_{0}}$

$$
\widehat{E}_{s_{0}} \simeq \operatorname{Spf} \widehat{\mathcal{O}}_{s_{0}} \times F .
$$

As $\pi$ is proper, we can apply Grothendieck's algebraization theorem [EGA, III $_{1}$, Théorème 5.4.1, p. 156] to obtain an isomorphism of $\widehat{\mathcal{O}}_{s_{0}}$-schemes

$$
\operatorname{Spec} \widehat{\mathcal{O}}_{s_{0}} \times_{S} E \stackrel{\simeq}{\longrightarrow} \operatorname{Spec} \widehat{\mathcal{O}}_{s_{0}} \times F .
$$

Using [EGA, IV $\mathrm{IV}_{3}$, Corollaire 8.8.2.5, p. 32] and the fact that $\widehat{\mathcal{O}}_{s_{0}}$ is a direct limit of flat $\mathcal{O}_{s_{0}}{ }^{-}$ algebras, it follows that there exist a flat morphism of finite type $U \rightarrow S$ whose image contains $s_{0}$ and an isomorphism of $U$-schemes

$$
E \times{ }_{S} U \stackrel{\simeq}{\longrightarrow} U \times F .
$$

Moreover, since $U \rightarrow S$ is open, for each $k$-point $s$ on the image of $U$, we have $F \simeq \pi^{-1}(s)$. Consequently, for any $s \in S(k)$ we have $\pi^{-1}(s) \simeq F$. 


\section{J.P. DOS SAntos}

Remark 6.3. The reader must have realized that in the preceding proof we used that $\widehat{\mathcal{O}}_{S, s_{0}}$ is a direct limit of flat $\mathcal{O}_{S, s_{0}}$-algebras of finite type. This result is an immediate consequence of a much more powerful one [Tei93]. We would be put in a hard position were we to demonstrate this weaker version from first principles.

\subsection{Proper stratified schemes obtained from $\mathcal{U}_{S}$}

Proposition 6.4. Let $\left(\pi: E \rightarrow S,\left\{\theta_{\nu}\right\}\right)$ be an object of $\operatorname{StrP}(S)$. Put $F:=\pi^{-1}\left(s_{0}\right)$ and assume that $\mathbf{A u t}_{k}(F)$ is affine. Then there exist a representation $\rho: \Pi(S) \rightarrow \mathbf{A u t}_{k}(F)$ and an isomorphism in $\operatorname{Str} \mathbf{P}(S)$ between $E$ and $\mathcal{U}_{S} \times{ }^{\rho} F$. Said differently, $E$ comes from the fundamental group scheme.

The proof will rely on a concept arising from differential geometry. Define the functor of frames

$$
\boldsymbol{I}:(\mathbf{S c h} / S)^{\mathrm{op}} \longrightarrow \text { Set }, \quad(U \rightarrow S) \longmapsto \operatorname{Isom}_{U}\left(F \times U, E \times{ }_{S} U\right) .
$$

To make the notation more suggestive, given $U \in \mathbf{S c h} / S$ and $a \in \operatorname{Hom}_{S}(U, \boldsymbol{I})$, we write

$$
\varphi_{a}: F \times U \stackrel{\sim}{\longrightarrow} E \times_{S} U
$$

to signify the obtained isomorphism of $U$-schemes. We shall find convenient to think of $\boldsymbol{I}$ as a functor $\mathbf{S c h}^{\text {op }} \rightarrow$ Set together with a morphism $\psi: \boldsymbol{I} \rightarrow S$, so that for each $a \in \boldsymbol{I}(U)=$ $\operatorname{Hom}_{k}(U, \boldsymbol{I})$, we have

$$
\varphi_{a}: F \times U \stackrel{\sim}{\longrightarrow} E \times_{S, \psi(a)} U
$$

It is also worth noting that $\boldsymbol{I}$ is a sheaf for the fpqc topology on Sch [TDTE, I, B.1, Théorème 2, p. 317] and that it possesses an action of the group scheme $\operatorname{Aut}_{k}(F)$ on its right. Explicitly, given $U \in \mathbf{S c h}$, a $U$-automorphism $\gamma: F \times U \rightarrow F \times U$, and $a \in \boldsymbol{I}(U)$, we define an element $a * \gamma$ by means of the isomorphism

$$
\varphi_{a * \gamma}: F \times U \stackrel{\gamma}{\longrightarrow} F \times U \stackrel{\varphi_{a}}{\longrightarrow} E \times \times_{S, \psi a} U .
$$

Before going any further, we remark that nothing guarantees that $\boldsymbol{I}$ is not the empty functor; it is by means of Lemma 6.2 that we avoid this.

To prove Proposition 6.4, we will show that $E$ is obtained from $\boldsymbol{I}$, and that $\boldsymbol{I}$ is a scheme induced from $\mathcal{U}_{S}$.

We begin with a sequence of exercises in descent theory and non-abelian cohomology. Let $S^{\prime} \rightarrow S$ be the morphism mentioned in the statement of Lemma 6.2. Then we have isomorphisms of functors

$$
\boldsymbol{I} \times{ }_{S} S^{\prime} \simeq \operatorname{Isom}_{S^{\prime}}\left(F \times S^{\prime}, E \times{ }_{S} S^{\prime}\right) \simeq \operatorname{Isom}_{S^{\prime}}\left(F \times S^{\prime}, F \times S^{\prime}\right)=\operatorname{Aut}_{k}(F) \times S^{\prime} .
$$

Moreover, letting $\mathbf{A u t}_{k}(F)$ act on itself on the right, these isomorphisms are all $\mathbf{A u t}_{k}(F)$ equivariant. As $\operatorname{Aut}_{k}(F)$ is affine by assumption, descent theory tells us that $\boldsymbol{I}$ is a scheme, affine over $S$. From the isomorphism $\boldsymbol{I} \times{ }_{S} S^{\prime} \simeq \mathbf{A u t}_{k}(F) \times S^{\prime}$, we conclude that $\psi: \boldsymbol{I} \rightarrow S$ is an $\mathbf{A u t}_{k}(F)$-torsor: $\psi$ is faithfully flat and affine due to [EGA, IV 2 , Propositions 2.5.1, p. 22 and 2.7.1, p. 29], and $\boldsymbol{I} \times{ }_{S} \boldsymbol{I} \simeq \boldsymbol{I} \times \operatorname{Aut}_{k}(F)$.

Let $q: F \times \boldsymbol{I} \rightarrow E$ be the composition of the "universal" isomorphism $\varphi_{\text {univ }}: F \times \boldsymbol{I} \cong E \times{ }_{S} \boldsymbol{I}$ with the projection $E \times{ }_{S} \boldsymbol{I} \rightarrow E$. If $U$ is a $k$-scheme and $a: U \rightarrow \boldsymbol{I}$ is a morphism, then $\varphi_{a}$ is just $\left(q \circ\left(\operatorname{id}_{F} \times a\right), \operatorname{pr}_{U}\right): F \times U \rightarrow E \times{ }_{S} U$. Therefore, for each $(y, a) \in(F \times \boldsymbol{I})(U)$, we have

$$
\varphi_{a}\left(y, \operatorname{id}_{U}\right)=\left(q(y, a), \operatorname{id}_{U}\right) .
$$




\section{Homotopy EXACT SEQUENCE}

Note that, in addition, $\pi(q(y, a))=\psi(a)$.

Lemma 6.5. (i) Let $\mathbf{A u t}_{k}(F)$ act on $F \times \boldsymbol{I}$ on the right by $(y, a) \cdot \gamma=\left(\gamma^{-1} y, a * \gamma\right)$. Then, for each $U \in \mathbf{S c h}$ and each $(y, a) \in(F \times \boldsymbol{I})(U)$, we have

$$
q[(y, a) \cdot \gamma]=q(y, a) .
$$

(ii) The morphism $q: F \times \boldsymbol{I} \rightarrow E$ induces an isomorphism

$$
\bar{q}:(F \times \boldsymbol{I}) / \mathbf{A u t}_{k}(F) \stackrel{\simeq}{\longrightarrow} E .
$$

Proof. (i) This follows directly from (6.3) defining the right action of $\mathbf{A u t}_{k}(F)$ on $\boldsymbol{I}$ and from the above characterization of $q$.

(ii) Endow $F \times \boldsymbol{I}$ with the action envisaged in part (i) and $E \times{ }_{S} \boldsymbol{I}$ with the action on $\boldsymbol{I}$. It follows that $\varphi_{\text {univ }}=\left(q, \operatorname{pr}_{\boldsymbol{I}}\right)$ is $\mathbf{A u t}_{k}(F)$-equivariant. We then obtain an isomorphism of quotient sheaves

$$
\bar{\varphi}_{\text {univ }}:(F \times \boldsymbol{I}) / \mathbf{A u t}_{k}(F) \stackrel{\simeq}{\longrightarrow}\left(E \times \times_{S} \boldsymbol{I}\right) / \mathbf{A u t}_{k}(F) .
$$

Due to the fact that formation of the sheaf associated with a presheaf commutes with fibre products [DG70, III.1.1.8, p. 287] and the fact that $\boldsymbol{I} \rightarrow S$ is an $\mathbf{A u t}_{k}(F)$-torsor, the quotient on the right-hand side above is simply $E$. Now, it is immediate that $\bar{q}$ equals $\bar{\varphi}_{\text {univ }}$.

We now show how to obtain a stratification on $\boldsymbol{I}$ starting from that on $E$. Fix a groupoid of Set $^{\text {sch }^{\text {op }}}$

$$
\mathfrak{P} \underset{p_{1}}{\stackrel{p_{0}}{\longrightarrow}} S, \quad \delta: \mathfrak{P} \times_{p_{0}, p_{1}} \mathfrak{P} \longrightarrow \mathfrak{P}, \quad \varepsilon: S \longrightarrow \mathfrak{P}, \quad s: \mathfrak{P} \longrightarrow \mathfrak{P},
$$

and admit the existence of an action of $\mathfrak{P}$ on the left of $\pi: E \rightarrow S$ :

$$
\theta: E \underset{\pi, S, p_{0}}{\times} \mathfrak{P} \stackrel{\sim}{\longrightarrow} \mathfrak{P} \underset{p_{1}, S, \pi}{\times} E .
$$

(See Definition 5.4.) Let $U \in \mathbf{S c h}, a \in \boldsymbol{I}(U)$, and $\partial \in \mathfrak{P}(U)$ be such that $p_{0}(\partial)=\psi(a)$ in $S(U)$. By means of $\varphi_{a}$ and the isomorphism $\theta_{\partial}$ of (5.3), we produce another isomorphism of $U$-schemes

$$
F \times U \stackrel{\varphi_{a}}{\longrightarrow} E \times_{S, \psi a} U=E \times_{S, p_{0} \partial} U \stackrel{\theta_{\partial}}{\longrightarrow} E \times_{S, p_{1} \partial} U .
$$

Hence, we arrive at an element

$$
\partial \bullet a \in \operatorname{Isom}_{U}\left(F \times U, E \times \times_{S, p_{1}(\partial)} U\right)=\boldsymbol{I}\left(U \stackrel{p_{1} \partial}{\longrightarrow} S\right)
$$

characterized by the condition that $\varphi_{\partial \bullet a}$ be the composition in (6.5). Note that

$$
\psi(\partial \bullet a)=p_{1}(\partial) \text {. }
$$

Lemma 6.6. The map

$$
(\partial, a) \in\left(\mathfrak{P} \times{ }_{p_{0}, S, \psi} \boldsymbol{I}\right)(U) \longrightarrow \boldsymbol{I}(U), \quad(\partial, a) \longmapsto \partial \bullet a
$$

defines an action of $\mathfrak{P}$ on $\psi: \boldsymbol{I} \rightarrow S$.

Proof. Let $\partial^{\prime}: U \rightarrow \mathfrak{P}$ be another arrow and assume $p_{1}(\partial)=p_{0}\left(\partial^{\prime}\right)$. Since $\theta_{\partial^{\prime}} \circ \theta_{\partial}=\theta_{\partial^{\prime} \partial}$, we conclude that $\partial^{\prime} \bullet(\partial \bullet a)=\partial^{\prime} \partial \bullet a$, so that axiom A3 of $\S 5.1$ holds. The verification of axiom A2 is trivial. Finally, that the arrow constructed in the statement is a natural transformation of functors is immediate. 


\section{J. P. DOS SANTOS}

Lemma 6.7. (i) The actions of $\mathfrak{P}$ and $\mathbf{A u t}_{k}(F)$ on $\boldsymbol{I}$ commute. More precisely, let $U \in \mathbf{S c h}$, $\partial \in \mathfrak{P}(U), \gamma \in \operatorname{Aut}_{k}(F)(U)$, and $a \in \boldsymbol{I}(U)$. Assume $p_{0}(\partial)=\psi(a)$. Then

$$
\partial \bullet(a * \gamma)=(\partial \bullet a) * \gamma .
$$

(ii) Endow $F \times \boldsymbol{I}$ with the left action of $\mathfrak{P}$ via $\boldsymbol{I}$. Then $q$ is equivariant.

Proof. (i) It is immediate to see that the composition

$$
F \times U \stackrel{\gamma}{\longrightarrow} F \times U \stackrel{\varphi_{a}}{\longrightarrow} E \times_{S, \psi a} U \stackrel{=}{\longrightarrow} E \times_{S, p_{0} \partial} \stackrel{\theta_{\partial}}{\longrightarrow} E \times_{S, p_{1} \partial} U
$$

defines $\varphi_{\partial \bullet(a * \gamma)}$ and $\varphi_{(\partial \bullet a) * \gamma}$; see (6.5) and (6.3).

(ii) This is again a triviality. Let $U \in \mathbf{S c h}, \partial \in \mathfrak{P}(U), \gamma \in \mathbf{A u t}_{k}(F)(U), y \in F(U)$, and $a \in \boldsymbol{I}(U)$. Assume $p_{0}(\partial)=\psi(a)$, and recall that in this case $\psi(\partial \bullet a)=p_{1}(\partial)$. For every $\left(e, \operatorname{id}_{U}\right) \in\left(E \times_{S, p_{0} \partial} U\right)(U)$ we have by definition $\theta_{\partial}\left(e, \operatorname{id}_{U}\right)=\left(\partial \cdot e, \operatorname{id}_{U}\right)$; see (5.3). Then, by definition of $q$, we have

$$
\left(\partial \cdot q(y, a), \operatorname{id}_{U}\right)=\theta_{\partial}\left(q(y, a), \operatorname{id}_{U}\right)=\theta_{\partial}\left(\varphi_{a}\left(y, \operatorname{id}_{U}\right)\right) .
$$

By definition of $\partial \bullet a$, the last term above is $\varphi_{\partial \bullet a}\left(y, \operatorname{id}_{U}\right)$. By $(6.4)$, this is just $\left(q(y, \partial \bullet a), \operatorname{id}_{U}\right)$.

Corollary 6.8. The left action of $\mathfrak{P}$ on $F \times \boldsymbol{I}$ considered in Lemma 6.7 descends to a left action of $\mathfrak{P}$ on the quotient $S$-scheme $(F \times \boldsymbol{I}) / \mathbf{A u t}_{k}(F)$. For this action, the isomorphism of $S$-schemes $\bar{q}$, defined in Lemma 6.5, is equivariant.

We now assume that $\mathfrak{P}=\widehat{P}_{S}$ is the formal diagonal groupoid of $S(\S 5.2)$; it acts on the left on $\pi: E \rightarrow S$ by means of its stratification; see Lemma 5.5. Then, $\psi: I \rightarrow S$ becomes a stratified affine scheme over $S$, so that the quasi-coherent $\mathcal{O}_{S}$-algebra $\mathcal{O}_{\boldsymbol{I}}$ associated with it becomes a stratified $\mathcal{O}_{S}$-module. In order to relate $\mathcal{O}_{\boldsymbol{I}}$ with the universal torsor $\mathcal{U}_{S}$, we need to show that the $\mathcal{D}_{S}$-module $\mathcal{O}_{\boldsymbol{I}}$ is a direct limit of elements in $\mathcal{D}_{S}-\mathbf{m o d}$. This will be hidden in the fact that every representation of an affine group scheme is a direct limit of finite-dimensional ones.

Endow $\boldsymbol{I} \times{ }_{S} \boldsymbol{I}$ with the obvious stratification and $\boldsymbol{I} \times \mathbf{A u t}_{k}(F)$ with the stratification coming from $\boldsymbol{I}$. It is straightforward to see that the canonical isomorphism

$$
\boldsymbol{I} \times \mathbf{A u t}_{k}(F) \stackrel{\simeq}{\longrightarrow} \boldsymbol{I} \times S \boldsymbol{I}, \quad(a, \gamma) \longmapsto(a, a * \gamma)
$$

is an isomorphism of stratified $S$-schemes. Such properties characterize what is sometimes called a stratified torsor. If we go by the terminology of [dSan07, 2.3.2(a)], this says that $\mathcal{O}_{\boldsymbol{I}}$ is a torsor algebra of the category $\mathcal{D}_{S}-$ Mod (but not necessarily of $\operatorname{Ind}\left(\mathcal{D}_{S}-\mathbf{m o d}\right)$ !). We are now capable of constructing a tensor functor

$$
\mathcal{L}_{\boldsymbol{I}}:\left\{\begin{array}{c}
\text { finite-dimensional } \\
\text { representations of } \mathbf{A u t}_{k}(F)
\end{array}\right\} \rightarrow \mathcal{D}_{S^{-}} \mathbf{\text { mod }}
$$

by imitating [Jan87, Part I, 5.8] or [dSan07, 2.3.2, p. 701].

Lemma 6.9. The stratified torsor $\psi: \boldsymbol{I} \rightarrow S$ is of the form $\mathcal{U}_{S} \times{ }^{\rho} \mathbf{A u t}_{k}(F)$ for a certain morphism $\rho: \Pi(S) \rightarrow \operatorname{Aut}_{k}(F)$.

Proof. We begin by constructing $\rho$. Since $\psi^{-1}\left(s_{0}\right)$ possesses a $k$-rational point (recall that $F:=\pi^{-1}\left(s_{0}\right)$ ), the composition of $\mathcal{L}_{\boldsymbol{I}}$ with $s_{0}^{*}: \mathcal{D}_{S^{-}} \mathbf{m o d} \rightarrow k-\bmod$ is naturally equivalent to the forgetful functor. By definition of $\Pi(S)$, it follows that $s_{0}^{*}$ factors through the category of finite-dimensional representations of $\Pi(S)$. Hence, Tannakian reconstruction of homomorphisms $\left[\mathrm{DM} 82,2.9\right.$, p. 130] allows us to say that $s_{0}^{*} \circ \mathcal{L}_{\boldsymbol{I}}$ is the restriction functor between representation categories associated with some homomorphism $\rho$. Now the proof can be finished following [Nor76, § 2] or [dSan07, 2.3.2]. 


\section{HOMOTOPY EXACT SEQUENCE}

Proof of Proposition 6.4. Just put together Lemma 6.9 and Corollary 6.8.

\section{Infinitesimal equivalence relations and stratifications}

We fix a noetherian separated scheme $B$. The category of separated noetherian $B$-schemes will be denoted by $\mathbf{S c h} / B$, that of noetherian formal separated $B$-schemes by $\mathbf{F S c h} / B$. The category $\mathbf{S c h} / B$ is considered as a full subcategory of $\mathbf{F S c h} / B$ following [EGA, I, 10.4.8, p. 186]. More importantly, [EGA, I, Proposition 10.6.2, p.189 and Corollaire 10.6.10, p. 192] show that Sch $/ B$ is a dense subcategory [ML98, X, $\S 6$, p. 245ff] of $\mathbf{F S c h} / B$, so that the restriction of the Yoneda functor $\mathbf{F S c h} / B \rightarrow \mathbf{S e t}^{(\mathbf{F S c h} / B)^{\mathrm{op}}}$ to $\mathbf{S c h} / B$ is full and faithful [ML98, X, $\S$ 6, Proposition 2, p. 246]. To lighten the notation we let $\mathbf{S c h} / B$ be the setting of all our constructions and arrows. In compliance with this, we usually omit references to $B$, for example, $\times=\times_{B}, \Omega_{Z}^{1}=\Omega_{Z / B}^{1}$, etc. Let $Z$ be a separated $B$-scheme of finite type.

\subsection{Notation concerning the sheaves of principal parts}

Let $P_{Z}^{\mu}$ denote the $\mu$ th infinitesimal neighbourhood of the diagonal in $Z \times Z\left(=Z \times{ }_{B} Z\right)$; this is just $\operatorname{Spec} \mathcal{P}_{Z}^{\mu}$ or $\left(|Z|, \mathcal{P}_{Z}^{\mu}\right)$, where $\mathcal{P}$ is as in [EGA, $\mathrm{IV}_{4}$, Définition 16.3.1, p. 14]. Moreover, following [EGA, $\mathrm{IV}_{4}, 16.3 .5$, p. 15], the scheme $\mathcal{P}_{Z}^{\mu}$ is always considered as an $\mathcal{O}_{Z}$-algebra or module, except when explicitly stated otherwise, via "the first projection"

$$
\mathcal{O}_{Z} \longrightarrow \mathcal{P}_{Z}^{\mu}, \quad a \longmapsto a \otimes 1 \text {. }
$$

The structure associated with the "second projection projection" will be denoted by

$$
\tau_{Z}^{\mu}: \mathcal{O}_{Z} \longrightarrow \mathcal{P}_{Z}^{\mu},
$$

or anything resembling such notation. This homomorphism should be thought of as associating with a function in $\mathcal{O}_{Z}$ its "Taylor series" or truncated "Taylor series". For convenience, given $a \in \mathcal{O}_{Z}$, we write

$$
d^{\mu}(a)=\tau^{\mu}(a)-a .
$$

Remark 7.1. This notation differs from that in [EGA, $\mathrm{IV}_{4}, 16.3 .5$, p. 15].

Instead of working with the schemes $P_{Z}^{\mu}$, we can use the formal completion $\widehat{P}_{Z}$ of $Z \times Z$ along the diagonal. This is a formal noetherian scheme [EGA, I, §10]. For purely algebraic purposes, it is interesting to regard $\widehat{P}_{Z}$ as the topological space $|Z|$ endowed with the sheaf of rings $\widehat{\mathcal{P}}_{Z}=\lim _{\mu} \mathcal{P}_{Z}^{\mu}$. As for its truncated version, $\widehat{\mathcal{P}}_{Z}$ (in [EGA, IV , Définition 16.3.1, p. 14], this is denoted by $\left.\mathcal{P}_{Z / B}^{\infty}\right)$ will be regarded as an $\mathcal{O}_{Z}$-algebra via $a \mapsto a \widehat{\otimes} 1$, and the "Taylor series" will be denoted by $\tau_{Z}$ or simply $\tau$. The ideal of $Z \hookrightarrow \widehat{P}_{Z}$ will be denoted by $\mathfrak{D}_{Z}$. We also write

$$
d(a)=\tau(a)-a,
$$

which is an element of $\mathfrak{D}_{Z}$. It should be remarked that this notation is not in conflict with the usual notation for a differential of a function, since $d(a)$ induces this differential upon passage to $\mathfrak{D}_{Z} / \mathfrak{D}_{Z}^{2} \simeq \Omega_{Z}^{1}$.

\subsection{Basic definitions}

Definition 7.2. Let $u_{0}, u_{1}: U \rightarrow Z \times Z$ be two $U$-points of $Z$. We say that $u_{0}$ is infinitesimally close to $u_{1}$, denoted by $u_{0} \equiv_{\infty} u_{1}$, if $u_{0 \text {,red }}=u_{1 \text {,red }}$. This defines an equivalence relation on $Z$ [DG70, III.1.2.4, p. 292] whose graph will be denoted by $(Z / B)_{\infty}$ or $Z_{\infty}$. 


\section{J.P. DOS SAntos}

As on a noetherian scheme $U$ the nilradical $\mathrm{Nil}_{U}$ is nilpotent, the functor $Z_{\infty}:(\mathbf{S c h} / B)^{\mathrm{op}} \rightarrow$ Set is represented by the formal scheme $\widehat{P}_{Z}$.

Definition 7.3. (a) An infinitesimal equivalence relation (i.e.r.) is a sub-presheaf $\Phi$ of $Z \times$ $Z:(\mathbf{S c h} / B)^{\text {op }} \rightarrow$ Set which is an equivalence relation and which coincides with $\Delta_{Z} \subseteq Z \times Z$ when evaluated at reduced schemes in $\mathbf{S c h} / B$. In other words, an infinitesimal equivalence relation is an equivalence relation on $Z$ which is weaker than $Z_{\infty}$.

(b) Let $\Phi$ and $\Phi^{\prime}$ be equivalence relations on $Z$ and $Z^{\prime}$, respectively. A morphism $h: Z \rightarrow Z^{\prime}$ interweaves $\Phi$ and $\Phi^{\prime}$ if, for every $U \in \mathbf{S c h} / B$, the map $h(U) \times h(U)$ sends $\Phi(U)$ into $\Phi^{\prime}(U)$. (In [SGA3, IV , 3.1.4] such an arrow is called "compatible".)

The category whose objects are pairs $(Z, \Phi)$ consisting of a scheme $Z \in \mathbf{S c h} / B$ and an infinitesimal equivalence relation on $Z$, and whose arrows are interweaving morphisms will be denoted by $\operatorname{IER}(B)$. Analogously, given an i.e.r. $\Phi$ in $\mathbf{I E R}(B)$, we write $\mathbf{I E R} / \Phi$ for the subcategory of $\mathbf{I E R}(B)$ of objects over $\Phi$, that is, $\mathbf{I E R} / \Phi:=\mathbf{I E R}(B)_{/ \Phi}$ in more standard notation.

Note that every $Z$ comes with two i.e.r.'s: the trivial one defined by the diagonal and denoted by $\Delta_{Z}$, and the full one, defined by $Z_{\infty}$.

Definition 7.4 (Compare to [Eke87, 1]). Let $\Phi$ be an infinitesimal equivalence relation on $Z$. We say that $\Phi$ is schematic if $\Phi:(\mathbf{S c h} / B)^{\text {op }} \rightarrow$ Set is represented by a formal closed subscheme of $\widehat{P}_{Z}$. We will sometimes write $\widehat{P}_{\Phi}$ to denote the formal closed subscheme associated with $\Phi$.

We recall from [EGA, I, 10.14] that the data of a closed formal subscheme of $\widehat{P}_{Z}$ amounts to a sheaf $\mathfrak{A}$ of (coherent) ideals of the sheaf of rings $\widehat{\mathcal{P}}_{Z}$. The formal scheme associated with $\mathfrak{A}$ will then be the (topologically) ringed space

$$
V(\mathfrak{A}):=\left(\text { support of } \widehat{\mathcal{P}}_{Z} / \mathfrak{A}, \text { restriction of } \widehat{\mathcal{P}}_{Z} / \mathfrak{A}\right) \text {. }
$$

By [EGA, I, Proposition 10.6.2, p. 189], the space $V(\mathfrak{A})$ is the direct limit of the schemes defined by the ideals

$$
\frac{\mathfrak{A}+\mathfrak{D}_{Z}^{\mu+1}}{\mathfrak{D}_{Z}^{\mu+1}} \subseteq \frac{\widehat{\mathcal{P}}_{Z}}{\mathfrak{D}_{Z}^{\mu+1}}=\mathcal{P}_{Z}^{\mu},
$$

and the morphism $V(\mathfrak{A}) \rightarrow \widehat{P}_{Z}$ is adic [EGA, I, 10.12]. If $\mathfrak{A} \subseteq \mathfrak{D}_{Z}$ is an ideal, then for $V(\mathfrak{A})$ to define a schematic equivalence relation, we only need

$$
\delta^{\#}(\mathfrak{A}) \subseteq \mathfrak{A} \widehat{\otimes}_{\mathcal{O}} \widehat{\mathcal{P}}_{Z}+\widehat{\mathcal{P}}_{Z} \widehat{\otimes}_{\mathcal{O}} \mathfrak{A} \text { and } s^{\#}(\mathfrak{A}) \subseteq \mathfrak{A},
$$

where $\delta: \widehat{P}_{Z} \times_{Z} \widehat{P}_{Z} \rightarrow \widehat{P}_{Z}$ and $s: \widehat{P}_{Z} \rightarrow \widehat{P}_{Z}$ are the morphisms of formal schemes considered in $\S 5.2$. In particular, the morphisms $\delta^{\#}$ and $s^{\#}$ pass to the ring

$$
\widehat{\mathcal{P}}_{\Phi}:=\widehat{\mathcal{P}}_{Z} / \mathfrak{A} \text {. }
$$

If $\Phi$ is a schematic equivalence relation defined by the ideal $\mathfrak{A}$, we write

$$
\mathcal{A}_{\mu}(\Phi):=\frac{\mathfrak{A}+\mathfrak{D}_{Z}^{\mu+1}}{\mathfrak{D}_{Z}^{\mu+1}} \quad \text { and } \quad \mathcal{P}_{\Phi}^{\mu}=\mathcal{P}_{Z}^{\mu} / \mathcal{A}_{\mu}(\Phi) \text {. }
$$

In other words,

$$
\left(|Z|, \mathcal{P}_{\Phi}^{\mu}\right)=\widehat{P}_{\Phi} \cap P_{Z}^{\mu}
$$

So, the question of how to find families of ideals $\mathcal{A}_{\mu} \subseteq \mathcal{P}_{Z}^{\mu}$ such that $\lim _{\leftarrow} \mathcal{A}_{\mu}$ defines a schematic equivalence relation is at hand. This is treated in the following example. 


\section{HоMOTOPY EXACT SEQUENCE}

Example 7.5 ([Miy87]). Let $B=\operatorname{Spec}(K)$ be the spectrum of a field of characteristic zero, and let $Z$ be a smooth $K$-scheme. Denote by $\mathcal{F}$ a subbundle of $\mathcal{D e r}_{Z / K}$ which is stable under the Lie bracket (an integrable distribution). Fixing a closed point $z \in Z$, we can find an affine open neighbourhood $U=\operatorname{Spec}(A)$ of $z$, etale coordinates $\left(x_{1}, \ldots, x_{n}\right): U \rightarrow \mathbb{A}^{n}$, and commuting vector fields $D_{1}, \ldots, D_{r}$ freely generating $\left.\mathcal{F}\right|_{U}$ such that $D_{i} x_{j}=\delta_{i j}$ for $1 \leqslant i, j \leqslant r$. (See the argument in the proof of Frobenius' theorem in the appendix of [CLN85].) From this arrangement, we can consider the formal flow of $\mathcal{F}$, which is the following homomorphism of $K$-algebras

$$
\exp (\boldsymbol{t} \cdot \boldsymbol{D}): A \longrightarrow A \llbracket t_{1}, \ldots, t_{r} \rrbracket, \quad \psi \longmapsto \sum_{\boldsymbol{j} \in \mathbb{N}^{r}} \boldsymbol{D}^{\boldsymbol{j}}(\psi) \cdot \frac{\boldsymbol{t}^{\boldsymbol{j}}}{\boldsymbol{j} !}
$$

The formal flow induces a formal action of $\widehat{\mathbb{G}}_{a, K}^{r}=\operatorname{Spf} K \llbracket \boldsymbol{t} \rrbracket$ on $U$. Define $\rho: A \otimes_{K} A \rightarrow A \llbracket \boldsymbol{t} \rrbracket$ by sending $\psi_{0} \otimes \psi_{1}$ to $\psi_{0} \cdot \exp (\boldsymbol{t} \cdot \boldsymbol{D})\left(\psi_{1}\right)$. Since the kernel of the multiplication $A \otimes_{K} A \rightarrow A$ is generated by elements of the form $1 \otimes \psi_{0}-\psi_{0} \otimes 1$, see for example [BO78, p. 2.2], it follows that $\rho$ induces a morphism of $A$-algebras

$$
\hat{\rho}: \widehat{\mathcal{P}}_{A} \longrightarrow A \llbracket \boldsymbol{t} \rrbracket
$$

Moreover, $\hat{\rho}$ is surjective because

$$
\exp (\boldsymbol{t} \cdot \boldsymbol{D})\left(x_{i}\right)=x_{i}+t_{i}, \quad i=1, \ldots, r .
$$

Write $\mathfrak{A}$ for $\operatorname{Ker}(\hat{\rho})$. Using the aforementioned action of $\widehat{\mathbb{G}}_{a, K}^{r}$, it is not difficult to verify that

$$
s^{\#}(\mathfrak{A}) \subseteq \mathfrak{A} \quad \text { and } \quad \delta^{\#}(\mathfrak{A}) \subseteq \mathfrak{A} \widehat{\otimes}_{A} \widehat{\mathcal{P}}_{A}+\widehat{\mathcal{P}}_{A} \widehat{\otimes}_{A} \mathfrak{A},
$$

where $\delta$ and $s$ are the morphisms used to define the diagonal formal groupoid; see $\S 5.2$.

Let

$$
\langle-,-\rangle: \mathcal{D}_{A}^{\mu} \otimes_{A} \mathcal{P}_{A}^{\mu} \longrightarrow A
$$

be the canonical pairing between the module of principal parts and the module of differential operators of order at most $\mu$ [EGA, $\mathrm{IV}_{4}$, Proposition 16.8.4, p. 41]. Since $\hat{\rho}$ maps the augmentation ideal $\mathfrak{D}_{A} \subseteq \widehat{\mathcal{P}}_{A}$ into $(\boldsymbol{t})$, we obtain a morphism of $A$-algebras

$$
\rho_{\mu}: \mathcal{P}_{A}^{\mu} \longrightarrow A[\boldsymbol{t}] /(\boldsymbol{t})^{\mu+1}
$$

which is none other than

$$
\xi \longmapsto \sum_{|i| \leqslant \mu}\left\langle D^{i}, \xi\right\rangle \frac{t^{i}}{i !}
$$

see the beginning of $\left[\mathrm{EGA}, \mathrm{IV}_{4}, 16.8\right]$ Write $\mathfrak{A}_{\mu}:=\operatorname{Ker}\left(\rho_{\mu}\right)$. Since

$$
\lim _{\mu} \rho_{\mu}: \lim _{\mu} \mathcal{P}_{A}^{\mu} \longrightarrow \underset{\longleftarrow}{\lim } A \llbracket \boldsymbol{t} \rrbracket /(\boldsymbol{t})^{\mu+1}
$$

is simply $\hat{\rho}$, we conclude that $\mathfrak{A}=\lim _{\longleftarrow} \mathfrak{A}_{\mu}$. Clearly, $\mathfrak{A}_{\mu}$ is simply $\Gamma\left(U, \mathcal{A}_{\mu}(\mathcal{F})\right)$, where

$$
\mathcal{A}_{\mu}(\mathcal{F})=\left\{\begin{array}{c}
\text { principal parts } \omega \in \mathcal{P}_{Z}^{\mu} \text { which are annihilated by all differential } \\
\text { operators of positive degree obtained by composition of fields in } \mathcal{F}
\end{array}\right\} .
$$

It follows that $\lim _{\mu} \mathcal{A}_{\mu}(\mathcal{F})$ defines a closed formal subscheme of $\widehat{P}_{Z}$ which is an infinitesimal equivalence relation due to (7.4).

Definition 7.6. Let $g: Z \rightarrow S$ be a morphism in $\mathbf{S c h} / B$, and let $\Phi$ be an equivalence relation on $Z$. We say that $\Phi$ is $S$-linear if $\Phi \subseteq Z \times{ }_{S} Z$. 


\section{J. P. DOS SANTOS}

If $\Phi$ is arbitrary, then its $S$-linearization is the equivalence relation $\Phi_{/ S}=\Phi \cap\left(Z \times_{S} Z\right)$ in $Z$; that is, two $U$-points $u_{0}, u_{1}$ of $Z$ are $\Phi_{/ S^{-}}$equivalent if and only if $u_{0} \equiv_{\Phi} u_{1}$ and $g\left(u_{0}\right)=g\left(u_{1}\right)$.

Remark 7.7. What we called an $S$-linear equivalence relation is called an equivalence relation compatible with $Z \rightarrow S$ in [SGA3, IV, 3.1.2]. The phrase "equivalence relation in $Z$ over $S$ " [SGA3, IV, 3.1.3] is also used.

Definition 7.8. Let $(Z, \Phi)$ and $(T, \Psi)$ be infinitesimal equivalence relations. Let $g: Z \rightarrow S$ and $h: T \rightarrow S$ be morphisms. On $Z \times_{S} T$ we have the i.e.r. defined on $U$-points by the following rule: two $U$-points $\left(z_{0}, t_{0}\right)$ and $\left(z_{1}, t_{1}\right)$ of $Z \times_{S} T$ are $\Phi \times_{S} \Psi$ equivalent if $z_{0} \equiv_{\Phi} z_{1}$ and $t_{0} \equiv_{\Psi} t_{1}$. To simplify the notation, when $\Psi$ is the trivial i.e.r. $\Delta_{T}$, we write $\Phi \times_{S} T$ instead of $\Phi \times_{S} \Delta_{T}$.

\subsection{Relation to stratifications}

DeFinition 7.9. Let $g: Z \rightarrow S$ be a morphism, and let $\Phi$ be an infinitesimal equivalence relation on $Z$. We say that $\Phi$ is complementary to $g$ if

$$
\Phi \cap\left(Z \times{ }_{S} Z\right) \subseteq \Delta_{Z},
$$

in other words, if $z_{0} \equiv_{\Phi} z_{1}$ and $g\left(z_{0}\right)=g\left(z_{1}\right)$ implies $z_{0}=z_{1}$.

Here is another way to say that an i.e.r. $\Phi$ in $Z$ is complementary to $g: Z \rightarrow S$. Let $p_{0}: \Phi \rightarrow Z$ be the composition of the inclusion $\Phi \subseteq Z \times Z$ with the first projection, let $\pi_{0}: S_{\infty} \rightarrow S$ be the first projection, and let $g_{\infty}: Z_{\infty} \rightarrow S_{\infty}$ be the obvious arrow of functors. Then $\Phi$ is complementary to $g$ if and only if the arrow

is a monomorphism.

$$
\Phi \stackrel{\left(p_{0}, g_{\infty}\right)}{\longrightarrow} Z \times_{S, \pi_{0}} S_{\infty}
$$

Definition 7.10. Let $g: Z \rightarrow S$ be a morphism of schemes and let $\Phi$ be an infinitesimal equivalence relation on $Z$. We say that $\Phi$ is totally complementary to $g$ if the arrow in (7.5) defines an isomorphism. if

Rephrasing Definition 7.10, we see that the i.e.r. $\Phi$ in $Z$ is totally complementary to $g: Z \rightarrow S$

TC1. the $S$-linearization $\Phi_{/ S}$ is trivial;

TC2. for any given pair $(z \in Z(U), s \in S(U))$ satisfying $g(z) \equiv_{\infty} s$, there exists a $\widetilde{z} \in Z(U)$ such that $\widetilde{z} \equiv_{\Phi} z$ and $g(\widetilde{z})=s$.

If we think of the condition $g(z) \equiv_{\infty} s$ as being "there exists a path from $g(z)$ to $s$ ", and of the condition $\widetilde{z} \equiv_{\Phi} z$ as "there exists a path in $Z$ contained in a leaf of $\Phi$ ", then condition TC2 affirms the possibility of lifting paths "horizontally". This is reminiscent of what Ehresmann calls a connection on a fibered space [Ehr50, 3, definition on p. 36]. However, this parallel is simply verbal, as totally complementary infinitesimal equivalence relations are just stratifications (see below).

Remark 7.11. The role played by the base scheme $B$ goes unmentioned just to simplify the notation. However, when studying restrictions of infinitesimal equivalence relations, its utility will become apparent. Whenever necessary, we will say that $\Phi$ is totally complementary to some morphism with respect to the base $B$.

To illustrate with a pertinent instance, we consider an i.e.r. $\Phi$ which is totally complementary to the $B$-morphism $g: Z \rightarrow S$. If $f: S \rightarrow T$ is another arrow of $B$-schemes, then $\Phi_{/ T}$ becomes totally complementary to $g$ with respect to the base $T$. 


\section{HOMOTOPY EXACT SEQUENCE}

The connection between this concept and stratifications is obtained as follows. Let $g: Z \rightarrow S$ be endowed with a stratification as explained in $\S 5$ :

$$
\alpha: Z \times{ }_{S} \widehat{P}_{S} \longrightarrow Z \text {. }
$$

(It is useful to remind the reader that we are following Convention 5.6 when forming fibre products involving principal parts.) We write $\alpha_{\mu}$ for the composition of $\alpha$ with the canonical morphism $Z \times{ }_{S} P_{Z}^{\mu} \rightarrow Z \times{ }_{S} \widehat{P}_{S}$. Let $\pi_{0}: Z \times Z \rightarrow Z$ be the first projection and set

$$
\beta_{\mu}:=\left(\operatorname{pr}_{Z}, \alpha_{\mu}\right) .
$$

From the definition of an action of $\widehat{P}_{S}$, the composition

$$
Z \times{ }_{S} P_{S}^{\mu} \stackrel{\beta_{\mu}}{\longrightarrow} Z \times Z \stackrel{\left(\pi_{0}, g \times g\right)}{\longrightarrow} Z \times_{S}(S \times S)
$$

is just

$$
\operatorname{id}_{Z} \times{ }_{S} \text { (natural inclusion) }: Z \times{ }_{S} P_{S}^{\mu} \longrightarrow Z \times{ }_{S}(S \times S) .
$$

Since $\beta_{\mu}$ factors through the closed embedding $P_{Z}^{\mu} \rightarrow Z \times Z$, what we just observed implies that $\beta_{\mu}$ is a section of

$$
\left(\pi_{0 \mu}, g_{\mu}\right): P_{Z}^{\mu} \longrightarrow Z \times_{S} P_{S}^{\mu}
$$

where we write $\left(\pi_{0 \mu}, g_{\mu}\right)$ to denote the restriction of $\left(\pi_{0}, g \times g\right)$ to $P_{Z}^{\mu}$. Passing to the limit, we get a morphism

$$
\beta: Z \times{ }_{S} \widehat{P}_{S} \longrightarrow \widehat{P}_{Z}
$$

which is a section of $\left(\pi_{0}, g_{\infty}\right): \widehat{P}_{Z} \rightarrow Z \times_{S} \widehat{P}_{S}$. It follows from the fact that $\beta_{0}=\mathrm{id}_{Z}$ and [EGA, I, Lemme 10.14.4, p. 210] that $\beta$ is a closed embedding of formal schemes. Write $\widehat{P}_{\Phi}$ for the image of $\beta$ in $\widehat{P}_{Z}$. The reader will have no difficulty in establishing that $\widehat{P}_{\Phi}$ is the graph of an infinitesimal equivalence relation. From the very construction, $\widehat{P}_{\Phi}$ is totally complementary to $g$.

On the other hand, interpreting $\mathbf{F S c h} / B$ as a full subcategory of $\mathbf{S e t}{ }^{(\mathbf{S c h} / B)^{\mathrm{op}}}$ (see the beginning of this section), it is not hard to show that if $g: Z \rightarrow S$ is totally complementary to $\Phi$, then $\Phi$ actually comes from a stratification. Indeed, given any $U \in \mathbf{S c h} / B$ and any pair $(z, \partial) \in\left(Z \times{ }_{S} \widehat{P}_{S}\right)(U)$, there exists a unique $(z, \alpha(\partial, z)) \in \Phi(U)$ which is taken by $\left(\pi_{0}, g_{\infty}\right)$ to $(z, \partial)$. The association $(\partial, z) \mapsto \alpha(\partial, z)$ defines an action of $\widehat{P}_{S}$ on $g: Z \rightarrow S$, which is equivalent to a stratification by Lemma 5.5 .

\section{Smooth schematic infinitesimal equivalence relations}

In this section, $B$ denotes a noetherian and separated scheme. We follow the flow of the notation introduced in $\S 7.1$, so that $\mathbf{S c h} / B$ is the category of separated noetherian $B$-schemes. We let $g: Z \rightarrow B$ be a separated morphism of finite type. For the sake of graphical simplicity, we will omit notational references to $B$ whenever the omission is harmless: in this spirit, $\widehat{\mathcal{P}}_{Z}=\widehat{\mathcal{P}}_{Z / B}$, $\mathfrak{D}_{Z}=\mathfrak{D}_{Z / B}$ (see $\S 7.1$ for this notation), $\Omega_{Z}^{1}=\Omega_{Z / B}^{1}$, etc.

\subsection{Basic objects}

Let $\Phi$ be a schematic infinitesimal equivalence relation on $Z$ cut out by the ideal $\mathfrak{A}$ of $\widehat{\mathcal{P}}_{Z}$. The sheaf of rings of the formal scheme $\widehat{P}_{\Phi}$ (the underlying space is simply $|Z|$ ) is denoted by $\widehat{\mathcal{P}}_{\Phi}$. The "Taylor expansion in the direction of $\Phi$ ",

$$
\tau_{\Phi}: \mathcal{O}_{Z} \longrightarrow \widehat{\mathcal{P}}_{\Phi}
$$




\section{J. P. DOS SANTOS}

will be the evident homomorphism of sheaves of algebras on $Z: \mathcal{O}_{Z} \stackrel{\tau}{\rightarrow} \widehat{\mathcal{P}}_{Z} \rightarrow \widehat{\mathcal{P}}_{\Phi}$, where $\tau$ is the morphism of sheaves of rings alluded to in $\S 7.1$. In like fashion, if the need presents itself, we will write

$$
d_{\Phi}(a):=\tau_{\Phi}(a)-a
$$

Note that $\tau_{\Phi}$ is a homomorphism of rings, while $d_{\Phi}$ is additive and satisfies

$$
d_{\Phi}(a \cdot b)=a \cdot d_{\Phi}(b)+b \cdot d_{\Phi}(a)+d_{\Phi}(a) \cdot d_{\Phi}(b) .
$$

We let $\mathfrak{D}_{\Phi}$ denote the image of $\mathfrak{D}_{Z}$ in $\widehat{\mathcal{P}}_{\Phi}$.

DeFinITION 8.1. The schematic infinitesimal equivalence relation $\Phi \hookrightarrow Z_{\infty}$ is differentially smooth of rank $r$ if $\mathfrak{D}_{\Phi} \subseteq \widehat{\mathcal{P}}_{\Phi}$ is a quasi-regular ideal [EGA, $\mathrm{IV}_{4}$, Définition 16.9.1, p. 46] and the locally free $\widehat{\mathcal{P}}_{\Phi} / \mathfrak{D}_{\Phi}=\mathcal{O}_{Z}$-module

$$
\Omega_{\Phi}:=\mathfrak{D}_{\Phi} / \mathfrak{D}_{\Phi}^{2},
$$

called henceforth the cotangent module of $\Phi$, is of rank $r$.

One important class of examples of differentially smooth i.e.r.'s is given by the totally complementary i.e.r.'s of Definition 7.10. Indeed, if $g: Z \rightarrow S$ is a smooth morphism of smooth schemes, then an infinitesimal equivalence relation $\Phi$ on $Z$ which is totally complementary to $g$ must be differentially smooth of $\operatorname{rank} \operatorname{dim} S / B$.

Recall that a more direct way to say that an ideal $\mathcal{J}$ of some locally noetherian formal scheme $\mathfrak{V}$ is quasi-regular is to say that the $\mathcal{O}_{\mathfrak{V}} / \mathcal{J}$-module

$$
\mathcal{G} r_{\mu}(\mathcal{J})=\mathfrak{J}^{\mu} / \mathfrak{J}^{\mu+1}
$$

is locally free of rank $\left(\begin{array}{c}r-1+\mu \\ \mu\end{array}\right)$; this can be easily deduced from [EGA, $\mathrm{IV}_{4}$, Corollaire 16.9.4, p. 47] and Nakayama's lemma. This allows us to prove the following.

Lemma 8.2. If $\Phi$ is differentially smooth of rank $r$ in the $B$-scheme of finite type $Z$, then for each $B^{\prime} \in \mathbf{S c h} / B$, the i.e.r. $\Phi \times B^{\prime}$ is differentially smooth of rank $r$ over $B^{\prime}$.

Proof. Let $\Phi$ be a differentially smooth i.e.r. of rank $r$ in $Z$. We fix some $\mu \in \mathbb{N}$ and let $P:=$ $P_{\Phi}^{\mu}$; denote by $\Delta$ the diagonal embedding $Z \rightarrow P$. It follows that for all $\nu<\mu$, the quotient $\mathcal{G r}_{\nu}(\Delta)=\mathfrak{D}_{\Phi}^{\nu} / \mathfrak{D}_{\Phi}^{\nu+1}$ is locally free of rank $\left(\begin{array}{c}r-1+\nu \\ \nu\end{array}\right)$ over $\mathcal{O}_{Z}$. Now, let $u: Z^{\prime} \rightarrow Z$ be a morphism in $\mathbf{S c h} / B$, and write $P^{\prime}=Z^{\prime} \times{ }_{Z} P$ and $\Delta^{\prime}=\mathrm{id}_{Z^{\prime}} \times{ }_{Z} \Delta \circ u$. From [EGA, IV $V_{4}$, Corollaire 16.2.4, p. 12], we conclude that

$$
\mathcal{G} r_{\nu}(\Delta) \otimes_{\mathcal{O}_{Z}} \mathcal{O}_{Z^{\prime}} \stackrel{\simeq}{\longrightarrow} \mathcal{G} r_{\nu}\left(\Delta^{\prime}\right)
$$

for all $\nu<\mu$. From the arbitrariness of $\mu$ and the above remark, we derive that the obvious morphism

$$
Z^{\prime} \longrightarrow Z^{\prime} \times{ }_{Z} \widehat{P}_{\Phi}=\underset{\mu}{\lim } Z^{\prime} \times{ }_{Z} P_{\Phi}^{\mu}
$$

is a quasi-regular embedding. Now, if $u: Z^{\prime} \rightarrow Z$ is just the projection from $Z^{\prime}=Z \times B^{\prime}$, it is not hard to deduce an isomorphism in $\mathbf{S e t}^{(\mathbf{S c h} / B)^{\text {op }}}$ between $Z^{\prime} \times{ }_{Z} \widehat{P}_{\Phi}=B^{\prime} \times \widehat{P}_{\Phi}$ and $\Phi \times B^{\prime}$ under which the closed embedding in (8.4) corresponds to the diagonal $Z \times B^{\prime} \rightarrow \Phi \times B^{\prime}$.

Whenever we study a differentially smooth i.e.r. $\Phi$, the cotangent module $\Omega_{\Phi}$ will play a prominent role. Before proceeding, we wish to attach a more familiar expression to it. Let $\mathfrak{A}$ be 
the ideal of $\Phi$ and denote by $\mathcal{A}_{1}(\Phi)$ the image of $\mathfrak{A}$ in $\mathcal{P}_{Z}^{1}=\mathcal{O}_{Z} \oplus \Omega_{Z}^{1}$. We have exact sequences

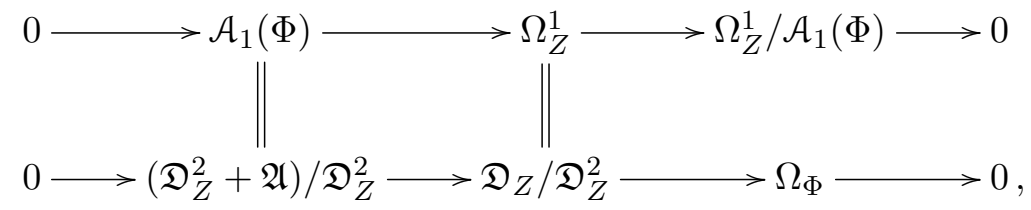

which lead to

$$
\Omega_{\Phi} \simeq \Omega_{Z}^{1} / \mathcal{A}_{1}(\Phi)
$$

We mention that this should be more familiar because of the following. Let $T_{\mathcal{F}} \subseteq T_{M}$ be the tangent bundle of a $r$-dimensional foliation on the differentiable manifold $M$. Let $A_{\mathcal{F}}$ be the sheaf of $C^{\infty}$ one-forms which annihilate the vectors in $T_{\mathcal{F}}$, that is, the kernel of $T_{M}^{*} \rightarrow T_{\mathcal{F}}^{*}$. This is usually called the conormal bundle of $\mathcal{F}$, as it can be identified with the dual of the normal bundle $N_{\mathcal{F}}=T_{M} / T_{\mathcal{F}}$; see $\left[\mathrm{MM} 03,1.3\right.$, p. 12]. The cotangent bundle $\Omega_{\mathcal{F}}:=T_{\mathcal{F}}^{*}$ of $\mathcal{F}$ is then isomorphic to $T_{M}^{*} / A_{\mathcal{F}}$. In particular, the rank of $\Omega_{\mathcal{F}}$ is the dimension of the leaves of the foliation $\mathcal{F}$.

\subsection{Local behaviour and transverse sections}

Let $\Phi$ be a differentially smooth infinitesimal equivalence relation on $Z$ of rank $r$, let $z$ be a point of $Z$, and let $b=g(z)$. We assume that $\boldsymbol{k}(b) \rightarrow \boldsymbol{k}(z)$ is bijective. As $\Omega_{Z}^{1}(z)$ is generated by elements of the form $d \psi(z)$, the equality $\boldsymbol{k}(b)=\boldsymbol{k}(z)$ allows us to find $x_{1}, \ldots, x_{r} \in \mathfrak{M}_{Z, z}$ such that the images of $d x_{1}, \ldots, d x_{r} \in \Omega_{Z, z}^{1}$ freely generate $\Omega_{\Phi, z}$. As $\mathfrak{D}_{\Phi}$ is a quasi-regular ideal of $\widehat{\mathcal{P}}_{\Phi}$, an application of [EGA, $\mathrm{IV}_{4}$, Corollaire 16.9.5, p. 47] gives $\left(d x_{1}, \ldots, d x_{r}\right)=\mathfrak{D}_{\Phi}$ near $z$, where we use the notation introduced in $\S 7.1$. Hence, near $z$, we have

$$
\mathcal{O}_{Z} \llbracket d x_{1}, \ldots, d x_{r} \rrbracket=\widehat{\mathcal{P}}_{\Phi},
$$

due to $\left[\mathrm{EGA}, \mathrm{IV}_{1}, 19.5 .4\right.$, p. 91].

Definition 8.3. Let $x_{1}, \ldots, x_{r} \in \mathfrak{M}_{Z, z}$ be functions such that the images of the differentials $d x_{1}, \ldots, d x_{r} \in \Omega_{Z, z}^{1}$ in $\Omega_{\Phi, z}$ form a basis of the latter. Then the closed subscheme $\Sigma=\left\{x_{1}=\right.$ $\left.\cdots=x_{r}=0\right\}$ is called a transverse section of $\Phi$ at $z$.

We observe that there are usually many transverse sections. If $\left\{x_{1}=\ldots=x_{r}=0\right\}$ is such a section and $\left(a_{i j}\right) \in \mathbf{G L}_{r}\left(\mathcal{O}_{Z, z}\right)$, then $\Sigma^{\prime}=\left\{x_{1}^{\prime}=\cdots=x_{r}^{\prime}=0\right\}$, where $x_{j}^{\prime}:=\sum_{i} a_{i j} \cdot x_{i}$, is another transverse section. However, finding an element $y \in \mathcal{O}_{Z, z}$ such that $d y$ belongs to the kernel of $\Omega_{Z, z}^{1} \rightarrow \Omega_{\Phi, z}$ is not a problem of linear algebra: such functions are first integrals.

For further reference we record a direct consequence of the Jacobian criterion [BLR90, $\S 2.2$, Proposition 7, p. 39].

Lemma 8.4. Assume that $Z \rightarrow B$ is smooth of relative dimension $n$ at $z$. Let $\Sigma$ be a transverse section of $\Phi$ at $z$. Then $\Sigma$ is smooth of relative dimension $c=n-r$ at $z$.

A transverse section induces a decomposition of $\Omega_{Z}^{1}$ as follows. Let $\Sigma=\left\{x_{1}=\cdots=x_{r}=0\right\}$ be a transverse section at $z \in Z$. If $y_{1}, \ldots, y_{l}$ are elements of $\mathcal{O}_{Z, z}$ such that

$$
d\left(\left.y_{1}\right|_{\Sigma}\right), \ldots, d\left(\left.y_{l}\right|_{\Sigma}\right)
$$

generate $\Omega_{\Sigma, z}^{1}$, then the conormal exact sequence

$$
\mathcal{N}_{\Sigma / Z, z} \rightarrow \Omega_{Z, z}^{1} \otimes \mathcal{O}_{\Sigma, z} \rightarrow \Omega_{\Sigma, z}^{1} \rightarrow 0
$$




\section{J. P. DOs SANTOS}

shows that $\Omega_{Z, z}^{1} \otimes \mathcal{O}_{\Sigma, z}$ is generated by (the images of) $d y_{1}, \ldots, d y_{l}$ and $d x_{1}, \ldots, d x_{r}$. Hence, by Nakayama's lemma,

$$
\Omega_{Z, z}^{1}=\sum_{i=1}^{r} \mathcal{O}_{Z, z} \cdot d x_{i}+\sum_{j=1}^{l} \mathcal{O}_{Z, z} \cdot d y_{j}
$$

\subsection{Formal behaviour}

Let $\Phi$ be a differentially smooth infinitesimal equivalence relation on $Z$ of rank $r$, and let $z$ be a point of $Z$ whose residue field $\boldsymbol{k}(z)$ coincides with $\boldsymbol{k}(g(z))$. We will concentrate on the "formal" behaviour of $\Phi$, and therefore assume $Z=\operatorname{Spec}(\mathcal{O})$ and $B=\operatorname{Spec}(\Lambda)$, and that there exist functions $x_{1}, \ldots, x_{r} \in \mathcal{O}$ vanishing on $z$ such that $d x_{1}, \ldots, d x_{r}$ generate $\mathfrak{D}_{\Phi}$; the existence of these was clarified in $\S 8.2$. In particular, $\Sigma=\left\{x_{1}=\cdots=x_{r}=0\right\}$ is a transverse section of $\Phi$ (Definition 8.3) at $z$.

Recall from $\S 5.2$ that we have a morphism of $\mathcal{O}$-algebras

$$
\delta: \widehat{\mathcal{P}}_{\mathcal{O}} \longrightarrow \widehat{\mathcal{P}}_{\mathcal{O}} \widehat{\otimes}_{\mathcal{O}} \widehat{\mathcal{P}}_{\mathcal{O}}
$$

which is employed to define the formal diagonal groupoid. By (7.2), the morphism $\delta$ passes to the quotient and defines a morphism of $\mathcal{O}$-algebras $\delta: \widehat{\mathcal{P}}_{\Phi} \rightarrow \widehat{\mathcal{P}}_{\Phi} \widehat{\otimes}_{\mathcal{O}} \widehat{\mathcal{P}}_{\Phi}$ fitting into the commutative diagram

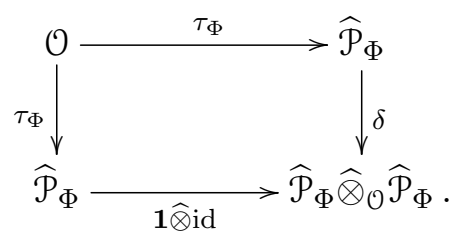

(We write 1 for the identity of $\widehat{\mathcal{P}}_{\mathcal{O}}$ or $\widehat{\mathcal{P}}_{\Phi}$.) Let $\Lambda \llbracket d_{1}, \ldots, d_{r} \rrbracket$ be the formal Hopf algebra associated with the completion of $\mathbb{G}_{a, \Lambda}^{r}$ at the identity. Using the identification $\widehat{\mathcal{P}}_{\Phi}=\mathcal{O} \llbracket d x_{1}, \ldots, d x_{r} \rrbracket$ made in (8.7), we can write

$$
\tau_{\Phi} y=\sum_{\alpha \in \mathbb{N}^{r}} \tau_{\Phi, \alpha}(y) \cdot d \boldsymbol{x}^{\alpha}
$$

where $\tau_{\Phi, \alpha}(y) \in \mathcal{O}$ and $d \boldsymbol{x}^{\alpha}=\left(d x_{1}\right)^{\alpha_{1}} \cdots\left(d x_{r}\right)^{\alpha_{r}}$. In terms of this notation, the composition $(\mathbf{1} \widehat{\otimes} \mathbf{i d}) \circ \tau_{\Phi}$ is simply

$$
y \longmapsto \mathbf{1} \widehat{\otimes} \sum_{\alpha \in \mathbb{N}^{r}} \tau_{\Phi, \alpha}(y) \cdot d \boldsymbol{x}^{\alpha}=\sum_{\alpha \in \mathbb{N}^{r}} \tau_{\Phi}\left(\tau_{\Phi, \alpha}(y)\right) \widehat{\otimes} d \boldsymbol{x}^{\alpha} .
$$

This fact and the identities $\delta\left(d x_{i}\right)=\mathbf{1} \widehat{\otimes} d x_{i}+d x_{i} \widehat{\otimes} \mathbf{1}$ show that $\tau_{\Phi}$ defines a co-action, in the category of topological $\Lambda$-algebras, of $\Lambda \llbracket d_{1}, \ldots, d_{r} \rrbracket$ on the discrete topological algebra $\mathcal{O}$. (The cautious reader will remark that we have left out the considerations concerning the behaviour of the co-identity; this is readily bypassed using the fact that $\tau(y) \equiv y \bmod \mathfrak{D}_{\mathcal{O}}$.) Clearly, this is reminiscent of the proof of Frobenius' theorem by means of $\mathbb{R}^{r}$-actions; see [CLN85, Appendix, Corollary 1, p. 181] and the pages that follow it. In our setting, a formal analogue of the Frobenius theorem, pointed out in [ESB05], is the following.

Proposition 8.5. Let $\mathcal{O}^{*}$ be the $(\boldsymbol{x})$-adic completion of $\mathcal{O}$, and write $\mathcal{O}(\Sigma)$ for the quotient $\mathcal{O} /(\boldsymbol{x})$. Then

$$
y \longmapsto \sum_{\alpha}\left\{\tau_{\Phi, \alpha}(y) \bmod (\boldsymbol{x})\right\} d_{1}^{\alpha_{1}} \cdots d_{r}^{\alpha_{r}}
$$


induces an isomorphism $\sigma: \mathcal{O}^{*} \rightarrow \mathcal{O}(\Sigma) \llbracket \boldsymbol{d} \rrbracket$ of adic rings. Moreover, if $\tau_{\Phi}^{*}: \mathcal{O}^{*} \rightarrow \mathcal{O}^{*} \llbracket d x_{1}, \ldots, d x_{r} \rrbracket$ is the obvious morphism, then the diagram

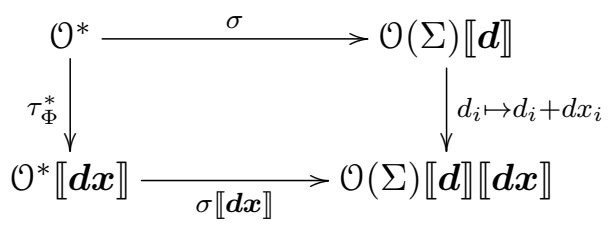

commutes. More geometrically, the formal scheme $\widehat{Z}_{/ \Sigma}$ is $\widehat{\mathbb{G}}_{a, \Lambda}^{r}$-equivariantly isomorphic to the product $\Sigma \times \widehat{\mathbb{G}}_{a, \Lambda}^{r}$.

Proof. The co-action of $\Lambda \llbracket \boldsymbol{d} \rrbracket$ on $\mathcal{O}$ extends to a co-action on $\mathcal{O}^{*}$, since $\tau_{\Phi}\left(x_{i}\right)=x_{i}+d x_{i}$ and the $(\boldsymbol{x}, \boldsymbol{d})$-adic completion of $\mathcal{O} \llbracket \boldsymbol{d} \rrbracket=\mathcal{O} \widehat{\otimes} \Lambda \llbracket \boldsymbol{d} \rrbracket$ is $\mathcal{O}^{*} \widehat{\otimes} \Lambda \llbracket \boldsymbol{d} \rrbracket$. Then [ESB05, Lemma 2.1, p. 115] can be applied since

$$
\Lambda \llbracket \boldsymbol{d} \rrbracket \longrightarrow \mathcal{O}^{*}, \quad d_{i} \longmapsto x_{i}
$$

is equivariant. See also [ESB05, Theorem 2.15].

Remark 8.6. One should observe, as is done in [ESB05], that this technique goes back to Zariski.

Corollary 8.7. For all $\mathcal{O}$-modules in sight, denote with an asterisk the (x)-adic completion. Let $\tau^{*}: \mathcal{O}^{*} \rightarrow \widehat{\mathcal{P}}_{\mathcal{O}}^{*}$ be the morphism obtained from $\tau$. If $\psi \in \mathcal{O}^{*}$ is such that $\sigma(\psi) \in \mathcal{O}(\Sigma)$, then $d^{*}(\psi):=\tau^{*}(\psi)-\psi \in \mathfrak{A} \cdot \widehat{\mathcal{P}}_{\mathcal{O}}^{*}$.

Proof. Use the diagram in the statement of Proposition 8.5 to obtain $\tau_{\Phi}^{*}(\psi)=\psi$.

\section{Tangency and invariance of subschemes}

Let $B$ be a noetherian and separated scheme. We work with the category $\mathbf{S c h} / B$ of $B$-schemes which are noetherian and separated, so that a "morphism", a "scheme", etc. should always be taken in $\mathbf{S c h} / B$. Continuing with the line of previous sections, we omit notational references to $B$ as much as possible, for example, $\times=\times_{B}, \mathcal{P}_{Z}^{\mu}=\mathcal{P}_{Z / B}^{\mu}$, etc. Let $Z$ be a scheme of finite type and let $\Phi$ be an infinitesimal equivalence relation on it.

\subsection{Tangency}

Let $\theta: Y \rightarrow Z$ be a closed embedding whose image is defined by the sheaf of ideals $I$. There is a canonical surjective homomorphism of sheaves of $\mathcal{O}_{Y}$-algebras, the "restriction of principal parts" [EGA, $\mathrm{IV}_{4},(16.4 .3 .3)$, p. 18]

$$
\theta^{*} \mathcal{P}_{Z}^{\mu} \longrightarrow \mathcal{P}_{Y}^{\mu}
$$

More precisely, (9.1) is constructed from the morphism of $\theta^{-1} \mathcal{O}_{Z}$-rings

$$
\theta^{-1} \mathcal{P}_{Z}^{\mu} \longrightarrow \mathcal{P}_{Y}^{\mu}
$$

which is used to define the "functoriality" morphism $P_{Y}^{\mu} \rightarrow P_{Z}^{\mu}$; see [EGA, $\mathrm{IV}_{4}, 16.2 .1$, p. 9 and (16.4.1.3), p. 16]. If $Z=\operatorname{Spec}(\mathcal{O}), Y=\operatorname{Spec}\left(\mathcal{O}_{0}\right)$, and $B=\operatorname{Spec}(\Lambda)$, then this is just induced by

$$
\mathcal{O}_{0} \otimes_{\mathcal{O}}\left(\left(\mathcal{O} \otimes_{\Lambda} \mathcal{O}\right) / \mathfrak{D}^{\mu+1}\right) \longrightarrow\left(\mathcal{O}_{0} \otimes_{\Lambda} \mathcal{O}_{0}\right) / \mathfrak{D}_{0}^{\mu+1}
$$

where $\mathfrak{D}$ and $\mathfrak{D}_{0}$ are the kernels of the corresponding multiplication homomorphism. The kernel of (9.1) is generated, as an $\mathcal{O}_{Y}$-module, by the image of $\tau_{Z}^{\mu}(I)$ [EGA, $\mathrm{IV}_{4}$, Proposition 16.4.20, p. 24]. 


\section{J.P. DOS SAntos}

Definition 9.1. Assume that $\Phi$ is schematic. For each $\mu \in \mathbb{N}$, let $\mathcal{A}_{\mu} \subseteq \mathcal{P}_{Z}^{\mu}$ be the sheaf of ideals of $\widehat{P}_{\Phi} \cap P_{Z}^{\mu}$. We say that a closed embedding $\theta: Y \hookrightarrow Z$ is $\nu$-tangent to $\Phi$ (respectively, tangent to $\Phi)$ relatively to $B$, or rel. $B$, if the natural composition

$$
\theta^{*} \mathcal{A}_{\mu} \longrightarrow \theta^{*} \mathcal{P}_{Z}^{\mu} \longrightarrow \mathcal{P}_{Y}^{\mu}
$$

is null for every $\mu \leqslant \nu$ (respectively, for every $\mu$ ).

A word of caution concerning the base scheme and the term "relatively to $B$ " is in order, and this is best illustrated by an example. Let $k$ be a field and put $B=\operatorname{Spec} k$. Let $g: \mathbb{A}^{2} \rightarrow \mathbb{A}^{1}$ be the projection on the first coordinate, and let $\Phi$ be the $\mathbb{A}^{1}$-linear i.e.r. in $\mathbb{A}^{2}$ defined by $\left(\mathbb{A}^{2} / \mathbb{A}^{1}\right)_{\infty}$ : two points $u_{0}, u_{1}: U \rightarrow \mathbb{A}^{2}$ are $\Phi$-equivalent if and only if $g u_{0}=g u_{1}$ and $\left.u_{0}\right|_{U_{\text {red }}}=\left.u_{1}\right|_{U_{\text {red }}}$. Therefore, $\mathbb{A}^{2} \subseteq \mathbb{A}^{2}$ is not tangent to $\Phi$ relatively to $B$, but is clearly tangent to $\Phi$ relatively to $\mathbb{A}^{1}$. This is because tangency is a property on the fibres over $B$, and not an absolute one.

In the special case where $\nu=1$, so that $\mathcal{P}_{Z}^{\mu}=\mathcal{O}_{Z} \oplus \Omega_{Z}^{1}$ [EGA, $\mathrm{IV}_{4}$, Définition 16.3.1, p. 14], the requirement that $\theta: Y \rightarrow Z$ be 1-tangent to $\Phi$ implies that $\Omega_{Y}^{1}$ is a quotient of $\left.\Omega_{\Phi}^{1}\right|_{Y}$, where $\Omega_{\Phi}$ is the cotangent sheaf of $\Phi$ defined in (8.6).

An equivalent definition of tangency consists in saying that $Y \subseteq Z$ is tangent to $\Phi$ rel. $B$ if for any $U \in \mathbf{S c h} / B$ and any pair of infinitesimally close points $y_{0}, y_{1} \in Y(U)$, we have $y_{0} \equiv_{\Phi} y_{1}$. Or, in yet another clothing, $Y$ is tangent to $\Phi$ if $Y \rightarrow Z$ interweaves (Definition 7.3) $Y_{\infty}$ and $\Phi$. These definitions, being completely expressed in terms of functors, apply to any infinitesimal equivalence relation on $Z$, schematic or not. The reason behind our choice of presenting this concept through Definition 9.1 will become clear in Section 10.

Remark 9.2. In the usual setting, a submanifold $S \rightarrow M$ is tangent to a foliation $\mathcal{F}$ if and only if $T_{x} S \subseteq T_{x} \mathcal{F}$ for every $x \in S$. This is possible if and only if $\left.\omega\right|_{S}=0$ for every $\omega \in \operatorname{Ann}(\mathcal{F})$.

\subsection{Invariant subschemes}

We now deal with a neighbouring notion. Throughout, $\theta: Y \rightarrow Z$ denotes a closed subscheme defined by a sheaf of ideals $I \subseteq \mathcal{O}_{Z}$.

Definition 9.3. We say that $\theta: Y \rightarrow Z$ is $\Phi$-invariant, or $\Phi$-saturated, if, for each $U \in \mathbf{S c h} / B$, the subset $Y(U) \subseteq Z(U)$ is saturated under the equivalence relation $\Phi(U)$.

We now take $\Phi$ schematic. Let us assume that $\theta: Y \rightarrow Z$ is $\Phi$-invariant; fix some $\nu \in \mathbb{N}$ and let $U$ be the inverse image of $Y$ by $p_{0}^{\nu}: P_{\Phi}^{\nu} \rightarrow Z$. Projecting to $Y$ defines a morphism $u_{0}: U \rightarrow Y$ and projecting to $P_{\Phi}^{\nu}$ and then composing with $p_{1}^{\nu}: P_{\Phi}^{\nu} \rightarrow Z$ defines a morphism $u_{1}: U \rightarrow Z$. Obviously, $u_{0} \equiv_{\Phi} u_{1}$ which, by invariance of $Y$, entails that $u_{1}$ factors through $\theta: Y \rightarrow Z$; this shows $\left(p_{0}^{\nu}\right)^{-1}(Y) \subseteq\left(p_{1}^{\nu}\right)^{-1}(Y)$.

On the other hand, assume that for each $\nu \in \mathbb{N}$, the second projection $p_{1}^{\nu}: P_{\Phi}^{\nu} \rightarrow Z$ restricted to $\left(p_{0}^{\nu}\right)^{-1}(Y)$ factors through $\theta: Y \rightarrow Z$. Let $u_{0}: U \rightarrow Y$ and $u_{1}: U \rightarrow Z$ be $\Phi$-close, so that, for some $\nu \in \mathbb{N}$, the map $\left(u_{0}, u_{1}\right): U \rightarrow P_{Z}^{\nu}$ factors through $P_{\Phi}^{\nu}$. As $u_{0}$ is a point of $Y$, it follows that $\left(u_{0}, u_{1}\right)$ factors through $\left(p_{0}^{\nu}\right)^{-1}(Y)$, so that $u_{1}=p_{1}^{\nu} \circ\left(u_{0}, u_{1}\right)$ must equally factor through $Y$. We state these findings in a more algebraic form, which will suit us later on.

Lemma 9.4. We assume that $\Phi$ is schematic. Let $I$ be an ideal of $\mathcal{O}_{Z}$, and let $Y$ be the closed subscheme cut out by $I$. Then $Y$ is $\Phi$-invariant if and only if

$$
\tau_{\Phi}(I) \subseteq I \cdot \widehat{\mathcal{P}}_{\Phi}
$$


The reader should note that for any ideal $I \subseteq \mathcal{O}_{Z}$, the inclusion $\tau_{\Phi}(I) \subseteq I \cdot \widehat{\mathcal{P}}_{\Phi}$ implies

$$
\tau_{\Phi}(I) \cdot \widehat{\mathcal{P}}_{\Phi}=I \cdot \widehat{\mathcal{P}}_{\Phi}
$$

since the switch automorphism

$$
s: \widehat{\mathcal{P}}_{\Phi} \longrightarrow \widehat{\mathcal{P}}_{\Phi}, \quad \psi_{0} \widehat{\otimes} \psi_{1} \longmapsto \psi_{1} \widehat{\otimes} \psi_{0}
$$

permutes $\tau_{\Phi}(I) \cdot \widehat{\mathcal{P}}_{\Phi}$ and $I \cdot \widehat{\mathcal{P}}_{\Phi}$.

One of the pleasing characteristics of invariance is that it is a "closed condition".

Proposition 9.5. Let $Y \subseteq Z$ be a closed subscheme of $Z$ cut out by the ideal $I$. Assume that there exists an open subset $Z^{\circ} \subseteq Z$ such that $Y^{o}=Y \cap Z^{\circ}$ is saturated under $\Phi$ and schematically dense in $Y$. Then $Y$ is saturated.

Proof. This is a local problem, so we assume $Z=\operatorname{Spec}(\mathcal{O})$. Moreover, we can suppose that $\mathcal{P}_{\Phi}^{\mu}$ is free over $\mathcal{O}$ on the basis

$$
d \boldsymbol{x}^{\alpha}=d x_{1}^{\alpha_{1}} \cdots d x_{r}^{\alpha_{r}}, \quad \alpha_{1}+\cdots+\alpha_{r} \leqslant \mu .
$$

It follows in particular that $I \cdot \mathcal{P}_{\Phi}^{\mu}=\bigoplus_{|\boldsymbol{\alpha}| \leqslant \mu} I \cdot d \boldsymbol{x}^{\alpha}$. Let $\psi \in I$. Then, $\tau_{\Phi}^{\mu}(\psi)=\sum_{\boldsymbol{\alpha}} \psi_{\boldsymbol{\alpha}} \cdot d \boldsymbol{x}^{\boldsymbol{\alpha}}$, and from the hypothesis, $\left(\psi_{\boldsymbol{\alpha}} \mid Y\right) \mid Y^{o}=0$. Since $Y^{o}$ is schematically dense in $Y$, we have $\psi_{\boldsymbol{\alpha}} \mid Y=0$. As $\mu$ is arbitrary, we arrive at the desired conclusion.

\subsection{Relation between the two concepts}

LEMmA 9.6. Let $Y \subseteq Z$ be a closed subscheme of $Z$ which is smooth of relative dimension $r$ overB. Assume that $\Phi$ is differentially smooth of rank $r$. Then $Y$ is tangent rel. $B$ if and only if $Y$ is invariant.

Proof. This is just a matter of counting ranks, applying Nakayama's lemma, and using [EGA, $\mathrm{IV}_{4}, 16.4 .20$, p. 24], which says that

$$
\mathcal{P}_{Y}^{\mu}=\frac{\mathcal{P}_{Z}^{\mu}}{I \cdot \mathcal{P}_{Z}^{\mu}+\tau^{\mu}(I) \cdot \mathcal{P}_{Z}^{\mu}} .
$$

It should be remarked that the assumption on smoothness is necessary. Just take the i.e.r. $\Phi$ on $\mathbb{A}_{\mathbb{C}}^{2}$ defined by $(x, y) \equiv\left(x^{\prime}, y^{\prime}\right)$ if and only if $x \equiv_{\infty} x^{\prime}$ and $y=y^{\prime}$ and note that $Y=\left\{y^{2}=0\right\}$ is invariant, but not tangent.

\subsection{The formal shape of invariant subschemes of maximal codimension}

We suppose $B=\operatorname{Spec}(\Lambda)$ and that $Z$ is the spectrum of a smooth $\Lambda$-domain $\mathcal{O}$ of relative dimension $n$. We let $\mathfrak{m}$ be a closed point of $Z$ whose residue field coincides with that of its image $\mathfrak{n}$ in $B$. We fix a differentially smooth i.e.r. $\Phi$ in $Z$ of rank $r$.

We now introduce a series of working properties, all of which can be guaranteed by passing to a smaller open subset of $Z$. We assume that $\Omega_{\Phi}$ (Definition 8.1) is freely generated by the image of differentials $d x_{1}, \ldots, d x_{r}$, where $x_{i} \in \mathfrak{m}$ for all $i$. The associated transverse section (see Definition 8.3) is

$$
\Sigma=\left\{x_{1}=\cdots=x_{r}=0\right\}
$$

this is a smooth $B$-scheme of relative dimension $c=n-r$, by Lemma 8.4. There is no serious impediment in assuming that

$$
\Omega_{\Sigma}^{1}=\bigoplus_{i=1}^{c} \mathcal{O}(\Sigma) \cdot d y_{i},
$$




\section{J. P. DOs SANTOS}

with $y_{i} \in \mathcal{O}(\Sigma)$ vanishing on $\mathfrak{m}$. So we include this hypothesis. Moreover, we assume that (8.8) actually gives

$$
\Omega_{Z}^{1}=\sum_{i=1}^{r} \mathcal{O} \cdot d x_{i}+\sum_{j=1}^{c} \mathcal{O} \cdot d \tilde{y}_{j},
$$

where $\tilde{y}_{i} \in \mathcal{O}$ lifts $y_{i}$. Let $y_{i}^{*} \in \mathcal{O}^{*}$ correspond to $y_{i}$ under the isomorphism

$$
\sigma: \mathcal{O}^{*} \longrightarrow \mathcal{O}(\Sigma) \llbracket \boldsymbol{d} \rrbracket, \quad \boldsymbol{d}=\left(d_{1}, \ldots, d_{r}\right),
$$

of Proposition 8.5.

Lemma 9.7. Let $\mathcal{O}^{\S}$, respectively $\mathfrak{m}^{\S}$, denote the $\mathfrak{m}$-adic completion of $\mathcal{O}$, respectively the maximal ideal of $\mathcal{O}^{\S}$. We shall consider $\mathcal{O}^{\S}$ as an $\mathcal{O}^{*}$-algebra in the obvious way.

(i) $y_{i}^{*} \in \mathfrak{m}^{\S}$.

(ii) Denote by $\mathfrak{n}$ the image of $\mathfrak{m}$ in $\operatorname{Spec}(\Lambda)$. Let $\boldsymbol{X}=\left(X_{1}, \ldots, X_{r}\right), \boldsymbol{Y}^{*}=\left(Y_{1}^{*}, \ldots, Y_{c}^{*}\right)$ be variables. Then the natural morphism $\hat{\Lambda}_{\mathfrak{n}} \llbracket \boldsymbol{Y}^{*}, \boldsymbol{X} \rrbracket \rightarrow \mathcal{O}^{\S}$ sending $\boldsymbol{X}$ to $\boldsymbol{x}$ and $\boldsymbol{Y}^{*}$ to $\boldsymbol{y}^{*}$ is an isomorphism.

(iii) We maintain the setting of the previous item. Let $\tau_{\Phi}^{\S}: \mathcal{O}^{\S} \rightarrow \mathcal{O}^{\S} \llbracket \boldsymbol{d} \boldsymbol{x} \rrbracket$ be the morphism obtained from $\tau_{\Phi}$ upon passage to the m-adic completion. Then, under the aforementioned isomorphism, $\tau_{\Phi}^{\S}$ corresponds to

$$
\hat{\Lambda}_{\mathfrak{n}} \llbracket \boldsymbol{Y}^{*}, \boldsymbol{X} \rrbracket \longrightarrow \hat{\Lambda}_{\mathfrak{n}} \llbracket \boldsymbol{Y}^{*}, \boldsymbol{X} \rrbracket \llbracket \boldsymbol{d} \boldsymbol{x} \rrbracket, \quad X_{i} \longmapsto X_{i}+d x_{i}, Y_{i}^{*} \longmapsto Y_{i}^{*} .
$$

Proof. (i) It is enough to show that $y_{i}^{*} \in \mathfrak{m} \cdot \mathcal{O}^{*}$. Since $\tau_{\Phi}(\psi) \equiv \psi \bmod (\boldsymbol{d} \boldsymbol{x})$ for all $\psi \in \mathcal{O}$, the very definition of $\sigma$ gives $\sigma\left(\tilde{y}_{i}\right) \equiv y_{i} \bmod (\boldsymbol{d})$. As $\sigma\left(x_{i}\right)=d_{i}$, it follows that

$$
\tilde{y}_{i} \equiv y_{i}^{*} \bmod (\boldsymbol{x}) \text {, }
$$

and this is enough to finish the proof.

(ii) We are assuming $\boldsymbol{k}(\mathfrak{n}) \cong \boldsymbol{k}(\mathfrak{m})$, so [EGA, IV 4 , Proposition 17.5.3, p. 68] guarantees that $\mathcal{O}^{\S}$ is isomorphic to a power series algebra over $\hat{\Lambda}_{\mathfrak{n}}$ in $n$ variables. Hence, we only need to show that the classes of $\boldsymbol{x}, \boldsymbol{y}^{*}$ generate the radical of $\mathcal{O}^{\S} / \mathfrak{r}\left(\hat{\Lambda}_{\mathfrak{n}}\right) \cdot \mathcal{O}^{\S}$. (From now on, $\mathfrak{r}$ refers to the maximal ideal, which equals the radical of a local ring.) From (9.4), it is sufficient to show that $\boldsymbol{x}, \tilde{\boldsymbol{y}}$ generate this radical. Since $\mathcal{O}^{\S} / \mathfrak{r}\left(\hat{\Lambda}_{\mathfrak{n}}\right) \cdot \mathcal{O}^{\S}$ is simply the completion of the local ring of the fibre of $Z \rightarrow B$ above $\mathfrak{n}$, namely $\mathcal{O}_{Z_{\mathfrak{n}}, \mathfrak{m}}=\mathcal{O}_{\mathfrak{m}} / \mathfrak{r}\left(\Lambda_{\mathfrak{n}}\right) \cdot \mathcal{O}_{\mathfrak{m}}$, the proof amounts to showing that $\boldsymbol{x}, \boldsymbol{y}$ generate the radical of $\mathcal{O}_{Z_{\mathfrak{n}}, \mathfrak{m}}$. This is obvious given (9.4), the isomorphims

$$
\begin{aligned}
\Omega_{\mathcal{O} / \Lambda}^{1} \otimes \boldsymbol{k}(\mathfrak{m}) & \cong \Omega_{Z_{\mathfrak{n}} / \boldsymbol{k}(\mathfrak{n})}^{1} \otimes \boldsymbol{k}(\mathfrak{m}) \\
& \cong \mathfrak{r}\left(\mathcal{O}_{Z_{\mathfrak{n}}, \mathfrak{m}}\right) / \mathfrak{r}\left(\mathcal{O}_{Z_{\mathfrak{n}}, \mathfrak{m}}\right)^{2},
\end{aligned}
$$

and Nakayama's lemma.

(iii) We begin by noting that the definition of $\tau_{\Phi}^{\S}$ is legitimate. For any $\psi \in \mathcal{O}$, we have $\tau_{\Phi}(\psi) \equiv \psi \bmod (\boldsymbol{d} \boldsymbol{x})$. Hence, $\tau_{\Phi}(\mathfrak{m}) \subseteq \mathfrak{m} \cdot \mathcal{O} \llbracket \boldsymbol{d} \boldsymbol{x} \rrbracket+(\boldsymbol{d} \boldsymbol{x})$, and this allows us to pass to completions. The rest follows immediately from the commutative diagram of Proposition 8.5, since $\sigma\left(x_{i}\right)=d_{i}$ and $\sigma\left(y_{i}^{*}\right)=y_{i}$.

We now apply ideas of Seidenberg [Sei67], see Theorem 1 of this reference, to study invariant subschemes of maximal codimension.

Lemma 9.8. Let $K$ be a field. Let $\boldsymbol{\xi}=\xi_{1}, \ldots, \xi_{r} ; \boldsymbol{\xi}^{\prime}=\xi_{1}^{\prime}, \ldots, \xi_{r}^{\prime}$; and $\boldsymbol{\eta}=\eta_{1}, \ldots, \eta_{c}$ be variables. Denote by $\alpha$ the ring homomorphism

$$
K \llbracket \boldsymbol{\xi}, \boldsymbol{\eta} \rrbracket \longrightarrow K \llbracket \boldsymbol{\xi}, \boldsymbol{\eta}, \boldsymbol{\xi}^{\prime} \rrbracket, \quad \xi_{i} \longmapsto \xi_{i}+\xi_{i}^{\prime}, \quad \eta_{i} \longmapsto \eta_{i},
$$




\section{Homotopy EXACT SEQUENCE}

and by $\iota: K \llbracket \boldsymbol{\xi}, \boldsymbol{\eta} \rrbracket \rightarrow K \llbracket \boldsymbol{\xi}, \boldsymbol{\eta}, \boldsymbol{\xi}^{\prime} \rrbracket$ the inclusion. Let $\mathfrak{K}$ be a proper ideal of $K \llbracket \boldsymbol{\xi}, \boldsymbol{\eta} \rrbracket$ satisfying ${ }^{\alpha} \mathfrak{K} \subseteq{ }^{\iota} \mathfrak{K}$. Then the following hold.

(i) $\mathfrak{K} \subseteq(\boldsymbol{\eta})$.

(ii) Assume that $\mathfrak{K}$ is primary and $\operatorname{ht}(\mathfrak{K}) \geqslant c$; then $\sqrt{\mathfrak{K}}=(\boldsymbol{\eta})$.

(iii) Assume only that $\operatorname{ht}(\mathfrak{K}) \geqslant c$; then $\mathfrak{K}$ is $(\boldsymbol{\eta})$-primary.

Proof. (i) Let $\psi_{1}(\boldsymbol{\xi}, \boldsymbol{\eta}), \ldots, \psi_{m}(\boldsymbol{\xi}, \boldsymbol{\eta})$ be generators of $\mathfrak{K}$ and let $A_{i j}\left(\boldsymbol{\xi}, \boldsymbol{\eta}, \boldsymbol{\xi}^{\prime}\right)$ be such that

$$
\psi_{i}\left(\boldsymbol{\xi}+\boldsymbol{\xi}^{\prime}, \boldsymbol{\eta}\right)=\sum_{j=1}^{m} \psi_{j}(\boldsymbol{\xi}, \boldsymbol{\eta}) \cdot A_{i j}\left(\boldsymbol{\xi}, \boldsymbol{\eta}, \boldsymbol{\xi}^{\prime}\right) .
$$

Then, making $\boldsymbol{\xi}=\mathbf{0}$ and $\boldsymbol{\eta}=\mathbf{0}$ in the above equation, we have

$$
\psi_{i}\left(\boldsymbol{\xi}^{\prime}, \mathbf{0}\right)=\sum_{j=1}^{m} \psi_{j}(\mathbf{0}, \mathbf{0}) \cdot A_{i j}\left(\mathbf{0}, \mathbf{0}, \boldsymbol{\xi}^{\prime}\right)=0 .
$$

(ii) We have ht $(\boldsymbol{\eta}) \leqslant c$. From the inclusion $\mathfrak{K} \subseteq(\boldsymbol{\eta})$, it follows that $\sqrt{\mathfrak{K}} \subseteq(\boldsymbol{\eta})$; as $\sqrt{\mathfrak{K}}$ is a prime of height at least $c$, we have $\sqrt{\mathfrak{K}}=(\boldsymbol{\eta})$.

(iii) Let $\mathfrak{q}$ be a primary ideal of $\mathfrak{K}$ and denote by $\mathfrak{p}$ its associated prime. Then ${ }^{\iota} \mathfrak{q}$ is a primary ideal with associated prime ${ }^{\iota} \mathfrak{p}$; see [Sei67, Lemma 2, p. 24]. Also, it is straightforward to see that the extension $\mathfrak{a} \mapsto{ }^{\iota} \mathfrak{a}$ takes intersections to intersections and is injective. Hence, if $\mathfrak{K}=\mathfrak{q}_{1} \cap \cdots \cap \mathfrak{q}_{e}$ is an irredundant primary decomposition of $\mathfrak{K}$ [AM94, Chapter 4], then ${ }^{\iota} \mathfrak{q}_{1} \cap \cdots \cap{ }^{\iota} \mathfrak{q}_{e}$ is an irredundant primary decomposition of ${ }^{\iota} \mathfrak{K}$. As the associated prime ideals are uniquely determined [AM94, Theorem 4.5], it follows that each associated prime of ${ }^{\iota} \mathfrak{K}$ is of the form ${ }^{\iota} \mathfrak{p}$ for some associated prime $\mathfrak{p}$ of $\mathfrak{K}$.

Let $\lambda: K \llbracket \boldsymbol{\xi}, \boldsymbol{\eta}, \boldsymbol{\xi}^{\prime} \rrbracket \rightarrow K \llbracket \boldsymbol{\xi}, \boldsymbol{\eta}, \boldsymbol{\xi}^{\prime} \rrbracket$ be the automorphism $\left(\boldsymbol{\xi}, \boldsymbol{\eta}, \boldsymbol{\xi}^{\prime}\right) \mapsto\left(\boldsymbol{\xi}+\boldsymbol{\xi}^{\prime}, \boldsymbol{\eta},-\boldsymbol{\xi}^{\prime}\right)$. Then we have $\lambda \circ \iota=\alpha, \lambda \circ \alpha=\iota$ and $\lambda \circ \lambda=$ id. Hence, the inclusion ${ }^{\alpha} \mathfrak{K} \subseteq{ }^{\iota} \mathfrak{K}$ is an equality. It follows that if $\mathfrak{p}$ is an associated prime of $\mathfrak{K}$, then ${ }^{\alpha} \mathfrak{p}={ }^{\lambda}\left({ }^{\iota} \mathfrak{p}\right)$ is an associated prime of ${ }^{\alpha} \mathfrak{K}={ }^{\iota} \mathfrak{K}$. Therefore, there exists another associated prime $\mathfrak{l}$ of $\mathfrak{K}$ for which ${ }^{\alpha} \mathfrak{p}={ }^{\iota} \mathfrak{l}$. Considering this equality modulo $\boldsymbol{\xi}^{\prime}$, we conclude that $\mathfrak{l}=\mathfrak{p}$. Thus, $\mathfrak{p}$ is a prime of height at least $c$ which satisfies ${ }^{\iota} \mathfrak{p}={ }^{\alpha} \mathfrak{p}$. The previous item shows that $\mathfrak{p}=(\boldsymbol{\eta})$, and we are done.

Corollary 9.9. Maintain the assumptions and notation of Lemma 9.7. Let $L \hookrightarrow Z$ be a closed subscheme passing through $\mathfrak{m}$ and cut out by an ideal $I \subseteq \mathcal{O}$. Assume that $L$ is flat over $B$, of codimension at least $c$ at $\mathfrak{m}$, and invariant under $\Phi$. Then, if $Z_{\mathfrak{n}}$ is the fibre of $Z$ above $\mathfrak{n}$, we have

$$
\sqrt{I \cdot \widehat{\mathcal{O}}_{Z_{\mathfrak{n}}, \mathfrak{m}}}=\left(\boldsymbol{y}^{*}\right) \text {. }
$$

In particular, if the fibre $L_{\mathfrak{n}}$ is reduced at $\mathfrak{m}$, then $L \rightarrow B$ is smooth at $\mathfrak{m}$.

Proof. It is a simple exercise to show that

$$
\text { ht } I \cdot \widehat{\mathcal{O}}_{Z \mathfrak{n}, \mathfrak{m}}=\text { ht } I \cdot \widehat{\mathcal{O}}_{Z, \mathfrak{m}}=\text { ht } I \cdot \mathcal{O}_{Z, \mathfrak{m}} \text {. }
$$

(Use [Mat89, Theorem 15.1, p. 116 and Theorem 17.4, p. 135] for the first equality, and [BouAC, VIII.3.4, Corollaire 4] for the second.) From this, it follows that $I \cdot \widehat{\mathcal{O}}_{Z_{\mathfrak{n}}, \mathfrak{m}}$ is of height at least $c$. The first part of the statement now ensues instantaneously from Lemma 9.7 and Lemma 9.8.

To prove the final statement, we recall that a theorem of Zariski and Nagata, see the examples and [BouAC, IX.4.4, Théorème 3], guarantees that once $\mathcal{O}_{L_{\mathfrak{n}}, \mathfrak{m}}$ is reduced, then so is $\widehat{\mathcal{O}}_{L_{\mathfrak{n}}, \mathfrak{m}}$. 


\section{J.P. DOS SANTOS}

Thus, $\boldsymbol{k}(\mathfrak{n}) \rightarrow \widehat{\mathcal{O}}_{L_{\mathfrak{n}}, \mathfrak{m}}$ is formally smooth, and $L_{\mathfrak{n}} \rightarrow \operatorname{Spec}(\boldsymbol{k}(\mathfrak{n}))$ is smooth at $\mathfrak{m}\left[\mathrm{EGA}, \mathrm{IV}_{4}\right.$, Proposition 17.5.3, p. 69], so that $L \rightarrow B$ is smooth at $\mathfrak{m}$ [EGA, $\mathrm{IV}_{4}$, Théorème 17.5.1, p. 67].

\section{The Hilbert scheme of subschemes tangent to an infinitesimal equivalence relation}

Let $B$ be a noetherian separated scheme. All ensuing constructions are made in $\mathbf{S c h} / B$, which is the category of separated and noetherian $B$-schemes (not necessarily of finite type). Let

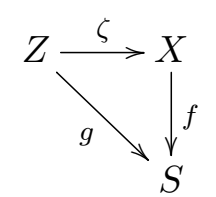

be a commutative diagram. We suppose that $f: X \rightarrow S$ is smooth of relative dimension $r$, geometrically connected, and proper. We also suppose that $g: Z \rightarrow S$ is quasi-projective and fix, tacitly, a $g$-ample invertible sheaf on $Z$. Under these assumptions, [TDTE, IV, $\S 4$ c, p. 19ff] guarantees that

$$
\mathbf{S c h} / S \longrightarrow \text { Set }, \quad T \longmapsto\left\{\begin{array}{l}
\text { closed subschemes } Y \hookrightarrow Z \times{ }_{S} T \text { flat over } T \\
\text { and isomorphic, via } \zeta \times \operatorname{id}_{T}, \text { to } X \times_{S} T
\end{array}\right\}
$$

is represented by a scheme

$$
\pi_{0}: H_{0} \longrightarrow S
$$

called the Weil restriction. Grothendieck's notation for $H_{0}$ is $\Pi_{X / S}(Z / X)$, see [TDTE, II, C.2] and [TDTE, IV, $\S 4 \mathrm{c}$ ], but $\mathfrak{R}_{X / S}(Z)$ can also be found in the literature, for example in [BLR90, $\S 7.6]$. The scheme $H_{0}$ is an open subscheme of the $S$-scheme $\operatorname{Hilb}(Z / S)$ and as such is locally of finite type and separated. In fact, $H_{0}$ is an open subscheme of the disjoint union

$$
\coprod_{\chi} \operatorname{Hilb}^{\chi}(Z / S)
$$

where $\chi \in \mathbb{Q}[\lambda]$ runs over all numerical polynomials (those which map $\mathbb{Z}$ into $\mathbb{Z}$ ) of degree $r=\operatorname{dim} f$. Given that each $\operatorname{Hilb}^{\chi}(Z / S)$ is a quasi-projective scheme, see [TDTE, IV, $\S 4 \mathrm{a}$ ] or [Nit05, Theorem 5.20], $H_{0}$ is a disjoint union of quasi-projective $S$-schemes. It is also worth noticing that if $\zeta$ is smooth, then so is $\pi_{0}$; this is a formal consequence of the "valuative criterion for smoothness"; see [BLR90, § 7.6, Proposition 5].

Remark 10.1. By calling $H_{0}$ a scheme, we have deviated from our initial convention: $H_{0}$ is not necessarily quasi-compact. This will cause no harm.

Notation 10.2. Let $\Phi$ be an infinitesimal $B$-linear equivalence relation on $Z$ such that $\Phi_{/ S}$ is differentially smooth of rank $r=\operatorname{dim} f$. We denote by $\mathcal{A}_{\mu}$ the sheaf of ideals in $\mathcal{P}_{Z / S}^{\mu}$ defining the closed formal subscheme $\Phi_{/ S} \cap P_{Z / S}^{\mu}$.

Let $y_{0} \rightarrow Z \times{ }_{S} H_{0}$ denote the universal closed subscheme. Composition of the canonical arrow $\left[\mathrm{EGA}, \mathrm{IV}_{4},(16.4 .3 .3)\right.$, p. 18]

with the obvious map

$$
\left.\mathcal{P}_{Z \times{ }_{S} H_{0} / H_{0}}^{\mu}\right|_{y_{0}} \longrightarrow \mathcal{P}_{y_{0} / H_{0}}^{\mu}
$$

$$
\left.\left.\left(\mathcal{A}_{\mu} \times{ }_{S} H_{0}\right)\right|_{y_{0}} \longrightarrow\left(\mathcal{P}_{Z / S}^{\mu} \times{ }_{S} H_{0}\right)\right|_{y_{0}}=\left.\mathcal{P}_{Z \times_{S} H_{0} / H_{0}}^{\mu}\right|_{y_{0}}
$$




\section{HOMOTOPY EXACT SEQUENCE}

(see [EGA, IV IV $_{4}$ Proposition 16.4.5, p. 19] to justify the identification made above) defines a homomorphism of $\mathcal{O}_{y_{0}}$-modules

$$
w_{\mu}:\left.\left(\mathcal{A}_{\mu} \times_{S} H_{0}\right)\right|_{y_{0}} \longrightarrow \mathcal{P}_{y_{0} / H_{0}}^{\mu} .
$$

Lemma 10.3. There exists a closed subscheme $H_{0}^{(\mu)} \subseteq H_{0}$ having the following property: an arrow $T \rightarrow H_{0}$ factors uniquely through $H_{0}^{(\mu)}$ if and only if the morphism obtained from (10.2)

$$
w_{\mu} \times H_{0} T:\left[\left(\mathcal{A}_{\mu} \times{ }_{S} H_{0}\right) \mid y_{0}\right] \times{ }_{H_{0}} T \longrightarrow \mathcal{P}_{y_{0} / H_{0}}^{\mu} \times_{H_{0}} T
$$

is null.

Proof. As $H_{0}$-schemes, $y_{0}$ and $X \times_{S} H_{0}$ are isomorphic. Hence, $\mathcal{P}_{y_{0} / H_{0}}^{\mu}$ is a locally free $\mathcal{O}_{y_{0}}$ module for any one of its two canonical structures [EGA, $\left.\mathrm{IV}_{4}, 16.10\right]$. A fortiori, $\mathcal{P}_{y_{0} / H_{0}}^{\mu}$ is flat over $H_{0}$. From the projectivity of $y_{0} \rightarrow H_{0}$, we are in the situation of [Nit05, Remark 5.9], and this results in what we expected. More precisely, there exists a linear scheme over $H_{0}$, call it $\mathbb{V} \rightarrow H_{0}$, which represents the functor

$$
T \in \mathbf{S c h} / H_{0} \longmapsto \operatorname{Hom}_{\mathcal{O}_{y_{0} \times H_{0}} T}\left(\left[\left.\left(\mathcal{A}_{\mu} \times_{S} H_{0}\right)\right|_{y_{0}}\right] \times_{H_{0}} T, \mathcal{P}_{y_{0} / H_{0}}^{\mu} \times_{H_{0}} T\right) .
$$

Since there are at least two homomorphisms of $\mathcal{O}_{y_{0}}$-modules

$$
\left.\left(\mathcal{A}_{\mu} \times{ }_{S} H_{0}\right)\right|_{y_{0}} \longrightarrow \mathcal{P}_{y_{0} / H_{0}}^{\mu}
$$

in sight, namely 0 and $w_{\mu}$, the morphism $\mathbb{V} \rightarrow H_{0}$ has two sections, and $H_{0}^{(\mu)}$ is their intersection.

From the surjectivity of the natural homomorphisms $\mathcal{A}_{\mu+1} \rightarrow \mathcal{A}_{\mu}$, it follows that the closed embedding $H_{0}^{(\mu+1)} \rightarrow H_{0}$ factors through $H_{0}^{(\mu)} \rightarrow H_{0}$.

Definition 10.4. The $S$-scheme $\cap_{\mu} H_{0}^{(\mu)}$ will be denoted by $H_{f}(Z, \Phi)$. The structure morphism to $S$ will be denoted by $\pi$.

Note that $H_{f}(Z, \Phi)$ is, as a closed subscheme of $H_{0}$, a disjoint union of quasi-projective schemes over $S$. Rewriting Lemma 10.3 in terms of flat families of closed subschemes, $H_{f}(Z, \Phi)$ represents the functor

$$
T \longmapsto\left\{\begin{array}{l}
\text { closed subschemes } Y \hookrightarrow Z \times_{S} T, \text { flat over } T \text { and isomorphic, } \\
\text { via } \zeta \times \operatorname{id}_{T}, \text { to } X \times_{S} T, \text { such that } \mathcal{A}_{\mu} \times_{S} T \rightarrow \mathcal{P}_{Y / T}^{\mu} \text { is null for all } \mu
\end{array}\right\} .
$$

Since the image of $\mathcal{A}_{\mu} \times{ }_{S} T$ in $\mathcal{P}_{Z / S}^{\mu} \times{ }_{S} T \cong \mathcal{P}_{Z \times{ }_{S} T / T}^{\mu}$ is just the ideal of $\Phi_{/ S} \times_{S} T$ in $P_{Z \times{ }_{S} T / T}^{\mu}$, $H_{f}(Z, \Phi)$ represents the functor

$$
T \longmapsto\left\{\begin{array}{l}
\text { closed subschemes } Y \hookrightarrow Z \times \times_{S} T, \text { flat over } T, \text { tangent rel. } T \\
\text { to } \Phi_{/ S} \times{ }_{S} T, \text { and isomorphic, via } \zeta \times \operatorname{id}_{T}, \text { to } X \times_{S} T
\end{array}\right\} .
$$

Or, in yet another interpretation, $H_{f}(Z, \Phi)$ represents the functor which parametrizes sections

$$
\sigma: X \times_{S} T \longrightarrow Z \times{ }_{S} T
$$

to

$$
\zeta \times{ }_{S} T: Z \times{ }_{S} T \longrightarrow X \times{ }_{S} T
$$




\section{J. P. DOS SANTOS}

which are tangent to the infinitesimal equivalence relation $\Phi_{/ S} \times_{S} T$. Using Lemma 9.6, we can also affirm that $H_{f}(Z, \Phi)$ represents

$$
T \longmapsto\left\{\begin{array}{l}
\text { closed subschemes } Y \hookrightarrow Z \times_{S} T, \text { flat over } T, \text { invariant under } \\
\Phi_{/ S} \times_{S} T, \text { and isomorphic, via } \zeta \times \operatorname{id}_{T}, \text { to } X \times_{S} T
\end{array}\right\} .
$$

With this reformulation, it is easier to establish the following result.

Theorem 10.5. Assume that $\zeta: Z \rightarrow X$ is smooth, proper, of relative dimension $c$, and that $\Phi_{/ S}$ is complementary to $\zeta$. Then the restriction of $\pi: H_{f}(Z, \Phi) \rightarrow S$ to each one of its connected components is projective.

Proof. We already know that $\pi$ is separated and locally of finite type, as $H_{f}(Z, \Phi)$ is a closed subscheme of the locally quasi-projective scheme $H_{0}$.

Let $T$ be an $S$-scheme which is the spectrum of a complete discrete valuation ring. The generic, respectively closed, point of $T$ will be denoted by $\gamma$, respectively $o$. We also include the hypothesis that $k:=\boldsymbol{k}(o)$ is algebraically closed. As is traditional, we abbreviate the operation of base-changing by decreeing $(*) \bullet=* \times_{S} \bullet$.

We consider a closed subscheme $Y_{\gamma} \subseteq Z_{\gamma}$ of $Z_{\gamma}$ which is saturated under $\Phi_{/ S} \times_{S} \gamma$ and isomorphic, via $\zeta_{\gamma}$, to $X_{\gamma}$. In particular, $Y_{\gamma}$ is an integral scheme. Let $Y_{T} \subseteq Z_{T}$ be a $T$-flat and closed subscheme of $Z_{T}$ which restricts to $Y_{\gamma}$ when intersected with the generic fibre $Z_{\gamma}=Z_{T} \backslash Z_{o}$ [EGA, $\mathrm{IV}_{2}$, Proposition 2.8.5, p. 35]. By construction, $Y_{\gamma} \rightarrow Y_{T}$ is schematically dense, so that $Y_{T}$ is integral. From Proposition 9.5, we know that $Y_{T}$ is saturated under $\Phi_{/ S} \times_{S} T$. We now prove that $Y_{T} \rightarrow X_{T}$ is quasi-finite [EGA, II, 6.2, p. 114ff], so that Zariski's main theorem [EGA, $\mathrm{III}_{1}$, Corollaire 4.4.9, p. 137] guarantees that $Y_{T} \stackrel{\sim}{\rightarrow} X_{T}$; the valuative criterion for properness, see [EGA, II, Théorème 7.3.8, p. 144 and Remarque 7.3.9i, p. 145], then concludes the proof.

To show that $Y_{T} \rightarrow X_{T}$ is quasi-finite, we pick a point $Q \in Y_{T}$; denote its image in $X_{T}$ by $P$. If $Q \in Y_{\gamma}$, then $\mathcal{O}_{X_{T}, P} \stackrel{\sim}{\rightarrow} \mathcal{O}_{Y_{T}, Q}$ by definition of $Y_{\gamma}$. Hence, we can assume that $Q$, respectively $P$, lies on the special fibre $Y_{o}$, respectively $X_{o}$. We assume that $Q$ is closed, or, equivalently, that $\boldsymbol{k}(P)=\boldsymbol{k}(Q)$ is the algebraically closed field $k$. Since the problem is now local, we write $Z_{T}=\operatorname{Spec}(\mathcal{O}), X_{T}=\operatorname{Spec}(A)$, and $T=\operatorname{Spec}(\Lambda)$. In order to simplify the notation, we let $\Psi$ denote the i.e.r. $\Phi_{/ S} \times_{S} T$. Recall that due to Lemma 8.2, the i.e.r. $\Psi$ is differentially smooth of rank $r=\operatorname{rel} . \operatorname{dim} .(A / \Lambda)$ over $T$.

Since $\Lambda \rightarrow A$ is smooth of relative dimension $r$, it is possible to find $x_{1}, \ldots, x_{r}$ such that the augmentation ideal $\mathfrak{D}_{A / \Lambda}$ of $\widehat{\mathcal{P}}_{A / \Lambda}$ is, near $P \in \operatorname{Spec}(A)$, generated by $d x_{1}, \ldots, d x_{r}$. As the ideal of $\widehat{P}_{Z_{T} / X_{T}}$ in $\widehat{P}_{Z_{T} / T}$ is generated by the image of the diagonal ideal $\mathfrak{D}_{A / \Lambda} \subseteq \widehat{\mathcal{P}}_{A / \Lambda}$ under the obvious morphism of topological rings

$$
\widehat{\mathcal{P}}_{A / \Lambda} \longrightarrow \widehat{\mathcal{P}}_{\mathcal{O} / \Lambda}
$$

the fact that $\Psi_{/ X_{T}}$ is trivial means that

$$
\mathfrak{D}_{A / \Lambda} \cdot \widehat{\mathcal{P}}_{\Psi}=\mathfrak{D}_{\Psi} .
$$

Since $\Psi$ is differentially smooth of relative dimension $r$, the quotient $\Omega_{\Psi}=\mathfrak{D}_{\Psi} / \mathfrak{D}_{\Psi}^{2}$ is then freely generated by the elements $d x_{1}, \ldots, d x_{r}$. Due to $\boldsymbol{k}(P)=k$, we can modify the $x_{i}$ to comply with the condition $x_{i}(P)=0$. A fortiori,

$$
\left\{x_{1}=\cdots=x_{r}=0\right\} \longleftrightarrow Z_{T}
$$

is a transverse section at $Q$ (Definition 8.3). Due to the smoothness assumption made on $\zeta$, we can apply [EGA, $\mathrm{IV}_{4}$, Proposition 17.5.3, p. 68], just as we did in Lemma 9.7, to identify $\widehat{\mathcal{O}}_{Z_{T}, Q}$ 
with $\Lambda \llbracket \boldsymbol{\xi}, \boldsymbol{\eta} \rrbracket$, where $\boldsymbol{\xi}=\left(\xi_{1}, \ldots, \xi_{r}\right)$ and $\boldsymbol{\eta}=\left(\eta_{1}, \ldots, \eta_{c}\right)$. Moreover, under this identification, $\xi_{i}$ corresponds to $x_{i}$.

Let $I \subseteq \mathcal{O}$ be the ideal of $Y_{T}$ in $Z_{T}$. As $Y_{T}$ is invariant under $\Psi$, flat over $T$, and of codimension at least $c$ at $Q$, we can use Corollary 9.9 to conclude that

$$
\sqrt{I \cdot \widehat{\mathcal{O}}_{Z_{o}, Q}}=(\boldsymbol{\eta}) \text {. }
$$

An important consequence of this equality is that for some integer $N$, the element $\eta_{i}^{N}$ belongs to $(\mathfrak{r}(\Lambda), I) \cdot \widehat{\mathcal{O}}_{Z_{T}, Q}$ for all $i$.

Since the residue field of $X_{T}$ at $P$, the image of $Q$, coincides with the residue field of $\Lambda$, an application of [EGA, IV IV $_{4}$ Proposition 17.5.3, p. 68] shows that it is possible to identify $\widehat{\mathcal{O}}_{X_{T}, P}$ with $\Lambda \llbracket \boldsymbol{\xi} \rrbracket$, where $\boldsymbol{\xi}=\left(\xi_{1}, \ldots, \xi_{r}\right)$. Moreover, the natural local morphism of complete local rings

$$
(\Lambda \llbracket \boldsymbol{\xi} \rrbracket=) \widehat{\mathcal{O}}_{X_{T}, P} \longrightarrow \widehat{\mathcal{O}}_{Z_{T}, Q}(=\Lambda \llbracket \boldsymbol{\xi}, \boldsymbol{\eta} \rrbracket)
$$

is the obvious inclusion of $\Lambda \llbracket \boldsymbol{\xi} \rrbracket$ in $\Lambda \llbracket \boldsymbol{\xi}, \boldsymbol{\eta} \rrbracket$. Hence, the radical of the local noetherian ring (the fibre local ring)

$$
\frac{\widehat{\mathcal{O}}_{Z_{T}, Q}}{\mathfrak{r}\left(\widehat{\mathcal{O}}_{X_{T}, P}\right) \cdot \widehat{\mathcal{O}}_{Z_{T}, Q}+I \cdot \widehat{\mathcal{O}}_{Z_{T}, Q}}=\frac{\widehat{\mathcal{O}}_{Z_{o}, Q}}{\mathfrak{r}\left(\widehat{\mathcal{O}}_{X_{o}, P}\right) \cdot \widehat{\mathcal{O}}_{Z_{o}, Q}+I \cdot \widehat{\mathcal{O}}_{Z_{o}, Q}}
$$

is generated by nilpotent elements. Therefore, this is a local Artin $k$-algebra of residue field $k$, and consequently a $k$-algebra of finite dimension. This means, by definition, that

$$
\widehat{\mathcal{O}}_{X_{T}, P} \longrightarrow \widehat{\mathcal{O}}_{Z_{T}, P} / I \cdot \mathcal{O}_{Z_{T}, P}=\widehat{\mathcal{O}}_{Y_{T}, Q}
$$

is quasi-finite [EGA, I, 7.4, p. 68ff], and therefore,

$$
\mathcal{O}_{X_{T}, P} \longrightarrow \mathcal{O}_{Y_{T}, Q}
$$

is quasi-finite. In conclusion, we have shown that $Y_{T} \rightarrow X_{T}$ is quasi-finite at any closed point. As the set of such points is open in $Y_{T}$ (a consequence of the main theorem; see [EGA, III 1 , Proposition 4.4.10, p. 137], we are done.

\section{Closed subschemes equivalent under an infinitesimal equivalence relation}

Let $B$ be a noetherian separated scheme. By $\mathbf{S c h} / B$ we understand the category of noetherian separated $B$-schemes and in what follows, unless explicitly stated otherwise, the words "morphism", "scheme", etc. should always be in this category. We endow $\mathbf{S c h} / B$ with the Zariski topology, so that the word "covering" will have that meaning. Finally, we fix $Z \in \mathbf{S c h} / B$ of finite type over $B$.

Let $Y_{0} \subseteq Z$ and $Y_{1} \subseteq Z$ be closed subschemes. For the sake of discussion, we will say that $Y_{0}$ is infinitesimally close to $Y_{1}$, and write $Y_{0} \equiv_{\infty} Y_{1}$, if they underlie the same topological space. Expanding this definition, we say that two closed embeddings $\theta_{0}: Y_{0} \rightarrow Z$ and $\theta_{1}: Y_{1} \rightarrow Z$ are infinitesimally close if the associated closed subschemes [EGA, I, 4.2.1, p. 122] are infinitesimally close. A more explicit way to say this is as follows: there exists a reduced scheme $R$, together with nilpotent closed embeddings $\omega_{0}: R \rightarrow Y_{0}$ and $\omega_{1}: R \rightarrow Y_{1}$, such that $\theta_{0} \circ \omega_{0}=\theta_{1} \circ \omega_{1}$.

Let $\Phi$ be an infinitesimal (B-linear) equivalence relation on $Z$.

Definition 11.1. Let $Y_{0}, Y_{1} \subseteq Z$ be closed subschemes. We say that $Y_{0} \leqslant_{\Phi} Y_{1}$ if, for each $U \in \mathbf{S c h} / B$ and each $y_{0} \in Y_{0}(U)$, there exist a covering $\tilde{U} \rightarrow U$ and a point $\tilde{y}_{1} \in Y_{1}(\tilde{U})$ which 


\section{J. P. DOS SANTOS}

is $\Phi$-equivalent to $y_{0} \mid \tilde{U} \in Y_{0}(\tilde{U})$. (Put in words, every $y_{0} \in Y_{0}$ is locally equivalent to some $y_{1}$ in $Y_{1}$.) We say that $Y_{0}$ and $Y_{1}$ are $\Phi$-equivalent, and write $Y_{0} \equiv Y_{1} \bmod \Phi$, if $Y_{0} \leqslant{ }_{\Phi} Y_{1}$ and $Y_{1} \leqslant{ }_{\Phi} Y_{0}$.

Obviously, the notion of $\Phi$-equivalence is an equivalence relation. Moreover, it is an infinitesimal equivalence relation in the following sense. Let Hilb be the scheme whose $U$-points correspond to the closed subschemes of $Z \times U$ which are proper and flat over $U$ (we assume that it exists). Fix some $u: U \rightarrow \mathbf{H i l b}$, and write $Y$ for the closed subscheme of $Z \times U$ corresponding to $u$. If $\omega: U_{\text {red }} \rightarrow U$ is the canonical nilpotent embedding, then $u \circ \omega: U_{\text {red }} \rightarrow$ Hilb defines the closed subscheme $Y^{\prime}=\left(\operatorname{id}_{Z} \times \omega\right)^{-1}(Y)$ of $Z \times U_{\text {red }}$. If assumptions are made to guarantee that $Y^{\prime}$ is reduced, we derive that $u \circ \omega$ actually corresponds to $Y_{\text {red }}$, which is a closed subscheme of $Z \times U_{\text {red }}$. In this setting, the following lemma, whose proof is omitted, explains the reason why the notion of $\Phi$-equivalence describes an i.e.r.

LEMMA 11.2. If $Y_{0} \equiv Y_{1} \bmod \Phi$, then $Y_{0, \text { red }}=Y_{1, \text { red }}$.

We now bring in the situation of Section 10 and let $H_{f}(Z, \Phi)$ be the scheme of Definition 10.4. Note that $H_{f}(Z, \Phi)$ is a $B$-scheme which comes with a natural morphism $\pi$ to $S$. (Of course, this just means that $H_{f}(Z, \Phi)$ is an $S$-scheme, but we want to emphasize that the morphism $\pi$ is to be regarded as an extra structure.) Consequently, for each morphism of $B$-schemes $u: U \rightarrow$ $H_{f}(Z, \Phi)$, we obtain a closed subscheme $Y(u)$ of $Z \times_{S, \pi u} U$. In the following definition, we identify, for any $u \in \operatorname{Hom}_{B}\left(U, H_{f}(Z, \Phi)\right)$, the scheme $Z \times_{S, \pi u} U$ as a closed subscheme of $Z \times U$.

DeFinition 11.3. Let $u_{0}$ and $u_{1}$ be morphisms of $B$-schemes from $U$ to $H_{f}(Z, \Phi)$. Let $Y_{0} \hookrightarrow$ $Z \times_{S, \pi u_{0}} U$ and $Y_{1} \hookrightarrow Z \times_{S, \pi u_{1}} U$ represent $u_{0}$ and $u_{1}$, respectively. We say that $u_{0} \equiv u_{1}$ $\bmod H_{\Phi}$, or $u_{0} \equiv u_{1} \bmod \Phi$ if confusion can be avoided, if, as closed subschemes of $Z \times U$, $Y_{0} \equiv Y_{1} \bmod \Phi \times U$. That is, the following two conditions must hold: (i) for each morphism of $U$-schemes $t_{0}: T \rightarrow Y_{0}$, there exist a covering $c: \widetilde{T} \rightarrow T$ and a $U$-morphism $\tilde{t}_{1}: \widetilde{T} \rightarrow Y_{1}$ such that $\tilde{t}_{1} \equiv t_{0} c \bmod \Phi \times U$, and (ii) the same as (i) but with $Y_{0}$ and $Y_{1}$ interchanged.

A direct application of Lemma 11.2, the fact that $X$ is smooth over $S$, and the discussion preceding it give the following result.

Lemma 11.4. The equivalence relation $H_{\Phi}$ is infinitesimal.

Remark 11.5. Let $U$ be a $B$-scheme, and let $Y_{0}, Y_{1} \subseteq Z \times U$ be closed subschemes. Assume $Y_{0} \leqslant \Phi \times U Y_{1}$, and let $t_{0}: T \rightarrow Y_{0}$ be a $U$-morphism. Then there exist a covering $c: \widetilde{T} \rightarrow T$ and a morphism $\tilde{t}_{1}: \widetilde{T} \rightarrow Y_{1}$ of $U$-schemes such that

$$
\tilde{t}_{1} \equiv t_{0} c \bmod \Phi \times U .
$$

However, there is no need to assume that $\tilde{t}_{1}$ is a morphism of $U$-schemes in the presence of (11.1): any morphism of $B$-schemes $\tilde{t}_{1}: \widetilde{T} \rightarrow Y_{1}$ satisfying (11.1) will, by definition of $\Phi \times U$, be a morphism of $U$-schemes. This means that in analyzing the equivalence relation $H_{\Phi}$ we have one less fact to check.

\section{Investigation of the adjointness property of $H_{f}(Z, \Phi)$}

We keep the assumptions and notation introduced in Section 10: $B$ is a noetherian and separated scheme; $\mathbf{S c h} / B$, which is the setting of our construction, is the category of noetherian separated schemes; $f: X \rightarrow S$ and $\zeta: Z \rightarrow X$ are morphisms such that $f$ is smooth of relative dimension $r$, 
geometrically connected, and proper; $g:=f \circ \zeta$ is quasi-projective; $\Phi$ is an i.e.r. such that $\Phi_{/ S}$ is differentially smooth (Definition 8.1) of rank $r$. We also assume that the infinitesimal equivalence relation $\Phi$ is schematic. The $S$-scheme $H_{f}(Z, \Phi)$ (Definition 10.4) will be abbreviated to $H$.

\subsection{The evaluation morphism}

By definition of $H$, there exists a morphism

$$
\Sigma=\left(\mathrm{ev}, \mathrm{pr}_{H}\right): X \times_{S, \pi} H \longrightarrow Z \times_{S, \pi} H
$$

which is a section of

$$
\zeta \times_{S} \operatorname{id}_{H}: Z \times_{S, \pi} H \longrightarrow X \times_{S, \pi} H .
$$

The morphism defined above,

$$
\text { ev : } X \times{ }_{S} H \longrightarrow Z,
$$

is henceforth called the evaluation morphism.

Proposition 12.1. The evaluation morphism (12.1) interweaves the infinitesimal equivalence relations $X_{\infty} \times{ }_{S} H_{\Phi}$ and $\Phi$.

Proof. Let $U \in \mathbf{S c h} / B$ and consider $U$-points $\left(x_{0}, u_{0}\right)$ and $\left(x_{1}, u_{1}\right)$ of $X \times_{S} H$. Put

$$
z_{i}=\operatorname{ev}\left(x_{i}, u_{i}\right) .
$$

We suppose $x_{0} \equiv x_{1} \bmod X_{\infty}$ and $u_{0} \equiv u_{1} \bmod H_{\Phi}$, and set out to show that $\left(z_{0}, \operatorname{id} d_{U}\right) \equiv$ $\left(z_{1}, \operatorname{id}_{U}\right) \bmod \Phi \times U$. Ensuing from $\left(x_{0}, u_{0}\right) \equiv_{\infty}\left(x_{1}, u_{1}\right)$ is the fact that $z_{0}$ and $z_{1}$ are infinitesimally close. Consequently, the two $U$-points $\left(z_{0}, \mathrm{id}_{U}\right)$ and $\left(z_{1}, \mathrm{id}_{U}\right)$ of $Z \times U$ are $(Z \times U / U)_{\infty}$-close.

The existence of $u_{i}$ defines, via $\Sigma$, a section

$$
\sigma_{i}: X \times_{S, \pi u_{i}} U \longrightarrow Z \times_{S, \pi u_{i}} U
$$

of $Z \times_{S, \pi u_{i}} U \rightarrow X \times_{S, \pi u_{i}} U$. By construction of $\Sigma$, it is clear that

$$
\sigma_{i}=\left(\mathrm{ev} \circ\left(\mathrm{id}_{X} \times u_{i}\right), \mathrm{pr}_{U}\right) .
$$

In particular, using

$$
z_{i}=\operatorname{ev} \circ\left(\operatorname{id}_{X} \times u_{i}\right) \circ\left(x_{i}, \operatorname{id}_{U}\right): U \longrightarrow Z,
$$

we conclude that

$$
\left(z_{i}, \operatorname{id}_{U}\right)=\sigma_{i} \circ\left(x_{i}, \operatorname{id}_{U}\right) .
$$

Let $Y_{0}$ and $Y_{1}$ be the closed subschemes of $Z \times U$ associated with $\sigma_{0}$ and $\sigma_{1}$, respectively. Due to the equivalence $u_{0} \equiv u_{1} \bmod H_{\Phi}$, we have $Y_{0} \equiv Y_{1} \bmod \Phi \times U$, so that it is possible to find a covering $c: \tilde{U} \rightarrow U$ and a point $\tilde{v}_{1} \in \operatorname{Hom}_{B}\left(\tilde{U}, Y_{1}\right)$ such that

$$
\tilde{v}_{1} \equiv\left(z_{0}, \operatorname{id}_{U}\right) \circ c \quad \bmod \Phi \times U .
$$

A fortiori,

$$
\tilde{v}_{1} \equiv\left(z_{0}, \operatorname{id}_{U}\right) \circ c \bmod (Z \times U / U)_{\infty},
$$

as $\Phi \times U \subseteq(Z \times U / U)_{\infty}$. Employing the equivalence

$$
\left(z_{0}, \operatorname{id}_{U}\right) \equiv\left(z_{1}, \operatorname{id}_{U}\right) \quad \bmod (Z \times U / U)_{\infty},
$$

it follows that

$$
\tilde{v}_{1} \equiv\left(z_{1}, \operatorname{id}_{U}\right) \circ c \quad \bmod (Z \times U / U)_{\infty} .
$$




\section{J. P. DOS SANTOS}

As $Y_{1}$ is tangent to $\Phi \times U$ and both $\tilde{v}_{1}$ and $\left(z_{1}, \operatorname{id}_{U}\right)$ are points of $Y_{1}$, we obtain

$$
\tilde{v}_{1} \equiv\left(z_{1}, \operatorname{id}_{U}\right) \circ c \bmod \Phi \times U .
$$

Using (12.2) and (12.3), we conclude that

$$
\left(z_{0}, \operatorname{id}_{U}\right) \circ c \equiv\left(z_{1}, \operatorname{id}_{U}\right) \circ c \bmod \Phi \times U .
$$

Due to the fact that $\Phi \times U$ is a sheaf, (12.4) guarantees the desired conclusion.

\subsection{Adjointness of $H_{f}(Z, \Phi)$}

We remind the reader that $H$ denotes the $S$-scheme $\pi: H_{f}(Z, \Phi) \rightarrow S$, and that $H_{\Phi}$ denotes the infinitesimal equivalence relation on $H$ introduced in Definition 11.3.

Let $(T, \Psi) \rightarrow\left(H, H_{\Phi}\right)$ be a morphism of IER/ $S_{\infty}$. (For the notation, see $\S 7.2$.) As ev (see $\S 12.1$ ) interweaves $H_{\Phi} \times_{S} X_{\infty}$ and $\Phi$ (Proposition 12.1), the composition

$$
\left(T \times_{S} X, \Psi \times_{S} X_{\infty}\right) \longrightarrow\left(H \times_{S} X, H_{\Phi} \times_{S} X_{\infty}\right) \stackrel{\text { ev }}{\longrightarrow}(Z, \Phi)
$$

interweaves $\Psi \times_{S} X_{\infty}$ and $\Phi$. We obtain a map

$$
\operatorname{Hom}_{S_{\infty}}\left(\Psi, H_{\Phi}\right) \longrightarrow \operatorname{Hom}_{X_{\infty}}\left(\Psi \times_{S} X_{\infty}, \Phi\right)
$$

which fits into the commutative diagram

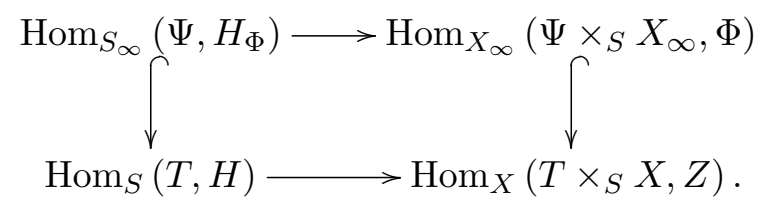

From the facts that $H$ is a closed subscheme of $H_{0}$ and that

$$
\operatorname{Hom}_{S}\left(T, H_{0}\right) \simeq \operatorname{Hom}_{X}\left(T \times_{S} X, Z\right)
$$

by definition of $H_{0}$ (see (10.1)), we conclude that arrow (12.5) is injective.

Let

$$
\sigma: T \times_{S} X \longrightarrow Z
$$

be an $X$-morphism which interweaves $\Psi \times{ }_{S} X_{\infty}$ and $\Phi$. In particular,

$$
\left(\mathrm{pr}_{T}, \sigma\right): T \times_{S} X \longrightarrow T \times{ }_{S} Z
$$

interweaves $T \times_{S}(X / S)_{\infty}\left(\subseteq \Psi \times_{S} X_{\infty}\right)$ and $T \times_{S} \Phi_{/ S}$, which means that $\left(\mathrm{pr}_{T}, \sigma\right)$ is tangent rel. $T$ to $T \times{ }_{S} \Phi_{/ S}$. This guarantees the existence of an arrow

$$
T \longrightarrow H
$$

of $S$-schemes which is mapped to $\sigma$ by the bottom horizontal arrow of (12.6).

Proposition 12.2. The arrow $T \rightarrow H$ generated above interweaves $\Psi$ and $H_{\Phi}$. That is, the arrow $\sigma \in \operatorname{Hom}_{X_{\infty}}\left(\Psi \times_{S} X_{\infty}, \Phi\right)$ belongs to the image of $\operatorname{Hom}_{S_{\infty}}\left(\Psi, H_{\Phi}\right)$.

Proof. Let $t_{0}, t_{1}: U \rightarrow T$ be $\Psi$-equivalent points, and write $s_{i}: U \rightarrow S$ to indicate the morphisms induced by $T \rightarrow S$. Using (12.7) we obtain two $U$-points of $H$. These are defined by the closed embeddings

$$
\left(\operatorname{pr}_{U}, \sigma \circ\left(t_{i} \times \operatorname{id}_{X}\right)\right): U \times_{s_{i}, S} X \longrightarrow U \times Z .
$$

Their images will be denoted by $Y_{i}$. (Note that $Y_{i}$ is in fact a closed subscheme of $U \times{ }_{s_{i}, S} Z$.) Our goal is to establish that $Y_{0}$ and $Y_{1}$ are $U \times \Phi$-equivalent. 


\section{HOMOTOPY EXACT SEQUENCE}

Let $\omega: U_{\text {red }} \hookrightarrow U$ be the reduced subscheme. Since $t_{0} \equiv_{\infty} t_{1}$, we know that $s_{0} \omega=s_{1} \omega$; we are then in a position to consider the following nilpotent embeddings:

$$
\omega_{i}=\omega \times \operatorname{id}_{X}: U_{\text {red }} \times{ }_{S} X \longrightarrow U \times_{s_{i}, S} X .
$$

To simplify the notation, let us write $Y_{i}$ instead of $U \times_{s_{i}, S} X$ in the remainder of the subsection.

Let $\rho: \widetilde{Y}_{0} \rightarrow Y_{0}$ be a Zariski covering of $Y_{0}$ with $\widetilde{Y}_{0}$ affine. We then arrive at a commutative diagram

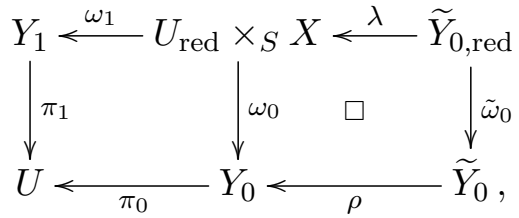

where $\pi_{1}$ is the natural projection, the rightmost square is cartesian, and the fibre product is identified with $\tilde{Y}_{0 \text {,red }}$ because $U_{\text {red }} \times_{S} X$ is reduced. As $Y_{1}$ is formally smooth over $U$, we obtain an arrow $\psi: \widetilde{Y}_{0} \rightarrow Y_{1}$ rendering commutative the diagram

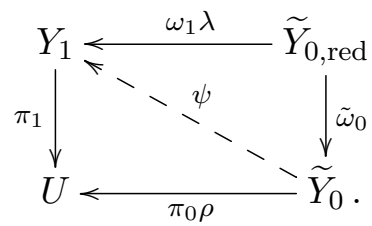

Let $V \in \mathbf{S c h} / B$, and consider an arbitrary $V$-point $w=\left(v, x_{0}\right): V \rightarrow Y_{0}=U \times_{s_{0}, S} X$. Let

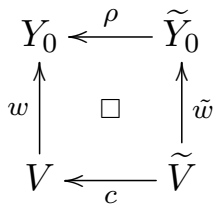

be the cartesian diagram deduced from it. Using $\psi$, we produce a $\widetilde{V}$-point $\psi \tilde{w}: \widetilde{V} \longrightarrow Y_{1}$. Due to the commutativity of diagrams (12.9) and (12.10), we have $\psi \tilde{w}=\left(v c, \tilde{x}_{1}\right)$ for some $\widetilde{V}$-point $\tilde{x}_{1}$ of $X$.

Since $t_{0}, t_{1}: U \rightarrow T$ are $\Psi$-equivalent, so are $t_{0}(v c)$ and $t_{1}(v c)$. As $\widetilde{V}_{\text {red }} \rightarrow \widetilde{V} \rightarrow \widetilde{Y}_{0}$ factors through $\tilde{\omega}_{0}: \widetilde{Y}_{0, \text { red }} \rightarrow \widetilde{Y}_{0}$ and $\operatorname{pr}_{X} \circ \omega_{0}=\operatorname{pr}_{X} \circ \omega_{1}$, it is not hard to conclude that $x_{0} c$ and $\tilde{x}_{1}$ are infinitesimally close. Hence the $\widetilde{V}$-points $\left(t_{0}(v c), x_{0} c\right)$ and $\left(t_{1}(v c), \tilde{x}_{1}\right)$ of $T \times_{S} X$ are $\Psi \times_{S} X_{\infty^{-}}$ equivalent. By the hypothesis on $\sigma$, the $\widetilde{V}$-points $\sigma\left(t_{0}(v c), x_{0} c\right)$ and $\sigma\left(t_{1}(v c), \tilde{x}_{1}\right)$ of $Z$ are $\Phi$ equivalent.

To summarize, we have started with an arbitrary $\left(v, z_{0}\right) \in U(V) \times Z(V)$ (here $z_{0}=\sigma \circ\left(t_{0} \times\right.$ $\left.\left.\operatorname{id}_{X}\right)\left(v, x_{0}\right)\right)$ belonging to $Y_{0}$ and obtained a Zariski covering $c: \widetilde{V} \rightarrow V$ and a $\widetilde{V}$-point $\left(v c, \tilde{z}_{1}\right)$ of $U \times Z$ belonging to $Y_{1}$ (here $\left.\tilde{z}_{1}=\sigma \circ\left(t_{1} \times \mathrm{id}_{X}\right)\left(v c, \tilde{x}_{1}\right)\right)$ and $U \times \Phi$-equivalent to $\left(v c, z_{0} c\right)$. This shows that $Y_{0} \leqslant_{U \times \Phi} Y_{1}$, and as the case $Y_{1} \leqslant_{U \times \Phi} Y_{0}$ is completely analogous, we conclude that $Y_{0}$ and $Y_{1}$ are $U \times \Phi$-equivalent.

Corollary 12.3. The morphism

$$
\begin{aligned}
\operatorname{Hom}_{S_{\infty}}\left(\Psi, H_{\Phi}\right) & \longrightarrow \operatorname{Hom}_{X_{\infty}}\left(\Psi \times_{S} X_{\infty}, \Phi\right) \\
(t: T \rightarrow H) & \longmapsto\left(\mathrm{ev} \circ\left(t \times_{S} \operatorname{id}_{X}\right): T \times_{S} X \rightarrow Z\right)
\end{aligned}
$$

is a bijection. 


\section{J. P. DOs SANTOS}

\subsection{Compatibility of $H$ with base change}

We consider a cartesian diagram

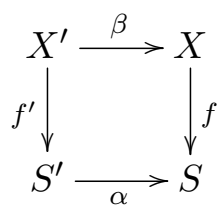

in $\mathbf{S c h} / B$. Denote $Z \times_{X} X^{\prime}$ by $Z^{\prime}$ and $\Phi \times_{X} X_{\infty}^{\prime}$ by $\Phi^{\prime}$. (Note that $\Phi_{/ S^{\prime}}^{\prime}=\Phi_{/ S} \times_{S} S^{\prime}$, which is differentially smooth of rank $r$.) Our goal is to compare the i.e.r. $H_{\Phi^{\prime}}$ in $H_{f^{\prime}}\left(Z^{\prime}, \Phi^{\prime}\right)$ with the i.e.r. $H_{\Phi} \times_{S} S_{\infty}^{\prime}$ in $H_{f}(Z, \Phi) \times{ }_{S} S^{\prime}$. In order to do this, one possible approach is to use the well-known fact that "right adjoints commute with limits", but due to technical inconveniences we opt to give a direct verification. In one way or another, this is a straightforward task.

If $\theta: T \rightarrow S^{\prime}$ is a morphism, we note that

$$
\rho:=\left(\mathrm{pr}_{T}, \beta \circ \mathrm{pr}_{X^{\prime}}\right): T \times_{S^{\prime}} X^{\prime} \longrightarrow T \times_{S} X
$$

is an isomorphism (the structure of $S$-scheme on $T$ is the obvious one); this permits the definition of the canonical bijection

$$
R: \operatorname{Hom}_{X}\left(T \times_{S} X, Z\right) \longrightarrow \operatorname{Hom}_{X^{\prime}}\left(T \times_{S^{\prime}} X^{\prime}, Z^{\prime}\right), \quad \varphi \longmapsto\left(\varphi \circ \rho, \operatorname{pr}_{X^{\prime}}\right) .
$$

This is functorial in $T \in \mathbf{S c h} / S^{\prime}$. Let $\Psi$ be an infinitesimal equivalence relation on $T$ and assume that $\varphi \in \operatorname{Hom}_{X}\left(T \times_{S} X, Z\right)$ interweaves $\Psi \times_{S} X_{\infty}$ and $\Phi$. Then, for a variable scheme $U$ and two $\Psi \times_{S^{\prime}} X_{\infty}^{\prime}$-equivalent $U$-points $\left(t_{0}, x_{0}^{\prime}\right)$ and $\left(t_{1}, x_{1}^{\prime}\right)$ of $T \times_{S^{\prime}} X^{\prime}$, it follows that

$$
R(\varphi)\left(t_{0}, x_{0}^{\prime}\right)=\left(\varphi\left(t_{0}, \beta\left(x_{0}^{\prime}\right)\right), x_{0}^{\prime}\right) \quad \text { and } \quad R(\varphi)\left(t_{1}, x_{1}^{\prime}\right)=\left(\varphi\left(t_{1}, \beta\left(x_{1}^{\prime}\right)\right), x_{1}^{\prime}\right)
$$

are $\Phi^{\prime}$-equivalent $U$-points of $Z^{\prime}$; therefore, $R(\varphi)$ interweaves $\Psi \times_{S^{\prime}} X_{\infty}^{\prime}$ and $\Phi^{\prime}$. Conversely, assume that $R(\varphi)$ interweaves $\Psi \times_{S^{\prime}} X_{\infty}^{\prime}$ and $\Phi^{\prime}$. Let $\left(t_{0}, x_{0}\right)$ and $\left(t_{1}, x_{1}\right)$ be any two given $\Psi \times_{S}$ $X_{\infty}$-equivalent $U$-points of $T \times_{S} X$ corresponding to $U$-points $\left(t_{0},\left[x_{0}, \theta\left(t_{0}\right)\right]\right)$ and $\left(t_{1},\left[x_{1}, \theta\left(t_{1}\right)\right]\right)$, respectively, of $T \times S_{S^{\prime}} X^{\prime}$. It is obvious that

$$
\left(t_{0},\left[x_{0}, \theta\left(t_{0}\right)\right]\right) \equiv\left(t_{1},\left[x_{1}, \theta\left(t_{1}\right)\right]\right) \quad \bmod \Psi \times_{S^{\prime}} X_{\infty}^{\prime},
$$

so that

$$
\left(\varphi\left(t_{0}, x_{0}\right),\left[x_{0}, \theta\left(t_{0}\right)\right]\right) \equiv\left(\varphi\left(t_{1}, x_{1}\right),\left[x_{1}, \theta\left(t_{1}\right)\right]\right) \bmod \Phi^{\prime},
$$

and, a fortiori, $\varphi\left(t_{0}, x_{0}\right) \equiv_{\Phi} \varphi\left(t_{1}, x_{1}\right)$. Thus we have shown that the dotted arrow in

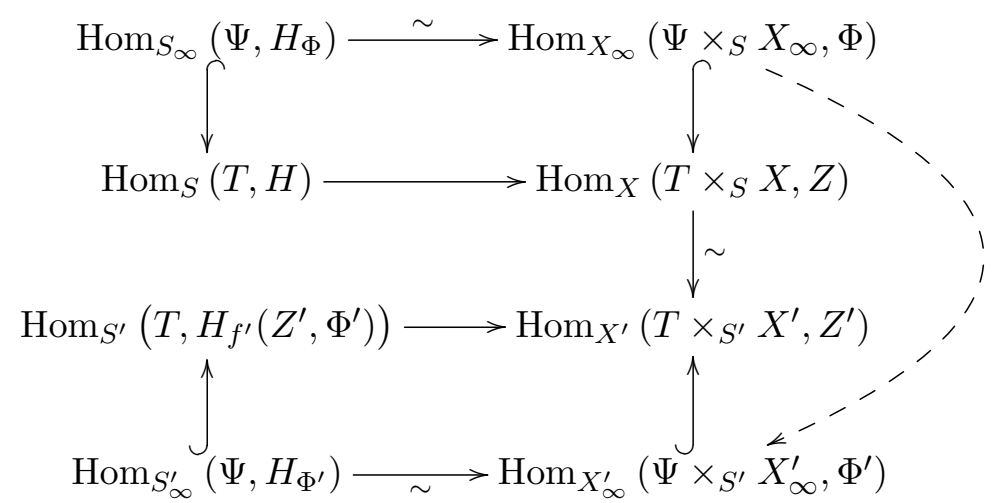

is bijective. Such a fact, when allied to the Yoneda lemma applied to IER/ $S_{\infty}^{\prime}$ and to the obvious 
isomorphism in $\mathbf{S e t}^{\left(\mathbf{I E R} / S_{\infty}^{\prime}\right)^{\text {op }}}$

$$
\operatorname{Hom}_{S_{\infty}}\left(\bullet, H_{\Phi}\right) \stackrel{\cong}{\longrightarrow} \operatorname{Hom}_{S_{\infty}^{\prime}}\left(\bullet, H_{\Phi} \times_{S} S_{\infty}^{\prime}\right),
$$

ensures the existence of an isomorphism of infinitesimal equivalence relations $H_{\Phi} \times_{S} S_{\infty}^{\prime} \cong H_{\Phi^{\prime}}$. These findings are collected in the following statement.

Corollary 12.4. Let $S^{\prime} \rightarrow S$ be a morphism of $\mathbf{S c h} / B$. Denote by $X^{\prime}$ the $S^{\prime}$-scheme $X \times_{S} S^{\prime}$, by $Z^{\prime}$ the $X^{\prime}$-scheme $Z \times_{X} X^{\prime}$, and by $\Phi^{\prime}$ the infinitesimal equivalence relation $\Phi \times_{X} X_{\infty}^{\prime}$ on $Z^{\prime}$. Then, there exists an isomorphism of infinitesimal equivalence relations over $S_{\infty}^{\prime}$ between $H_{\Phi} \times_{S} S_{\infty}^{\prime}$ and $H_{\Phi^{\prime}}$.

An afterthought. The application of the Yoneda lemma employed above can profit from further remarks, since $\left(H, H_{\Phi}\right)$ is not really an object of IER/ $S_{\infty}$. For $\left(H, H_{\Phi}\right)$ to belong to $\mathbf{I E R}(B)$, according to the convention of Subsection 7.2, the scheme $H$ should be quasi-compact. With this in mind, we introduce $\mathbf{S} \mathbf{c h}^{\prime} / B$, the category of locally noetherian separated schemes whose connected components are quasi-compact. Accompanying $\mathbf{S} \mathbf{c h}^{\prime} / B$ is the category $\mathbf{I E R}(B)$, whose definition is obtained by interchanging $\mathbf{S c h} / B$ and $\mathbf{S} \mathbf{c h}^{\prime} / B$ in Definition 7.3. But this is not yet what we need, and we introduce $c \mathbf{I E R}(B)$, the full subcategory of $\mathbf{I E R}(B)$ consisting of those equivalence relations which respect disjoint unions. We define $c \mathbf{I E R}^{\prime}(B)$ analogously. It is then easily proved that the evident functor $c \mathbf{I E R}(B) \rightarrow c \mathbf{I E R}^{\prime}(B)$ is full and dense (in the sense of [ML98, X.6, p. 246]) From that, the dual of [ML98, X.6, Proposition 2, p. 246] gives us the necessary Yoneda lemma. That $\left(H, H_{\Phi}\right)$ in fact belongs to $c \mathbf{I E R}(B)$ follows directly by using that $\Phi$, being schematic, belongs to $c \mathbf{I E R}(B)$.

\section{Conditions for the equivalence relation on $H_{f}(Z, \Phi)$ to come from a stratification}

Let $B$ be a noetherian separated base scheme, and let $\mathbf{S c h} / B$ be the category of noetherian separated $B$-schemes. All arrows and objects are in $\mathbf{S c h} / B$. Let

$$
Z \stackrel{\zeta}{\longrightarrow} X \stackrel{f}{\longrightarrow} S
$$

be smooth morphisms of smooth schemes. Denote the composition $f \circ \zeta$ by $g$ and the relative dimension of $f$ by $r$. Let $\Phi$ be a $B$-linear i.e.r. in $Z$ which is totally complementary to $\zeta$ (Definition 7.10); that is, it comes from a $B$-linear stratification of $\zeta$. (In particular, $\Phi$ is differentially smooth of rank $\operatorname{dim} X / B$.) Our goal here is to derive Corollary 13.10 below. This corollary is a translation of the following.

Proposition 13.1 (Parallel transport). Let $u, u^{\prime}: U \rightarrow S$ be infinitesimally close. Let $Y$ be a closed subscheme of $Z \times{ }_{S, u} U$ which is tangent to $\Phi_{/ g} \times_{S, u} U$ rel. $U$ and which is sent isomorphically, via $\zeta \times_{S, u} \operatorname{id}_{U}$, to $X \times_{S, u} U$. Then, there exists a unique closed subscheme $Y^{\prime} \subseteq Z \times_{S, u^{\prime}} U$ which is

(i) $\Phi \times U$-equivalent to $Y$;

(ii) tangent to $\Phi_{/ g} \times_{S, u^{\prime}} U$ rel. $U$; and

(iii) sent isomorphically, via $\zeta \times_{S, u^{\prime}} \operatorname{id}_{U}$, to $X \times_{S, u^{\prime}} U$.

We begin by showing that property (ii) follows from property (i). First, it is sufficient to prove that $Y^{\prime}$ is tangent to $\Phi \times U$ rel. $U$, since $Y^{\prime}$ is a closed subscheme of $Z \times_{S, u^{\prime}} U$. Second, we have the following result. 


\section{J. P. DOs SANTOS}

Lemma 13.2. Let $Y \subseteq Z \times U$ and $Y^{\prime} \subseteq Z \times U$ be closed subschemes which are equivalent modulo $\Phi \times U$. Assume that $Y$ is tangent to $\Phi \times U$ rel. $U$. Then $Y^{\prime}$ is tangent to $\Phi \times U$ rel. $U$.

Proof. We write $Z_{U}=Z \times U$ and $\Phi_{U}=\Phi \times U$. Let $t_{0}^{\prime}, t_{1}^{\prime} \in \operatorname{Hom}_{U}(T, Y)$ be infinitesimally close. Since $Y$ and $Y^{\prime}$ are equivalent modulo $\Phi_{U}$, there exist a covering $c: \widetilde{T} \rightarrow T$ and $\widetilde{T}$ points $\tilde{t}_{0}, \tilde{t}_{1} \in \operatorname{Hom}_{U}\left(\widetilde{T}, Y^{\prime}\right)$ such that $t_{i}^{\prime} \circ c \equiv \tilde{t}_{i} \bmod \Phi_{U}$. Since $\Phi_{U}$ is infinitesimal, $\tilde{t}_{0}$ and $\tilde{t}_{1}$ are infinitesimally close points in $Y$. Due to the tangency assumption made on $Y$, we have $\tilde{t}_{0} \equiv \tilde{t}_{1} \bmod \Phi_{U}$. Hence, $t_{0}^{\prime} \circ c \equiv t_{1}^{\prime} \circ c \bmod \Phi_{U}$. Because $\Phi_{U}$ is a sheaf, it follows that $t_{0}^{\prime} \equiv t_{1}^{\prime}$ $\bmod \Phi_{U}$

Continuing with the peeling off of minor assertions made in Proposition 13.1, we now show that "uniqueness" also comes without much effort. Since $\Phi$ is totally complementary to $\zeta$ (Definition 7.10), $\Phi_{/ g}$ is also totally complementary to $\zeta$ with respect to $S$ (see Remark 7.11), so $\Phi_{/ g}$ is differentially smooth of $\operatorname{rank} \operatorname{dim} X / S=r$ over $S$. Now, let $Y^{\prime}$ be as in Proposition 13.1. From properties (ii) and (iii) and Lemma 9.6, it follows that $Y^{\prime}$ is invariant under $\Phi_{/ g} \times_{S, u^{\prime}} U$. Consequently, $Y^{\prime}$ is also invariant under $\Phi_{/ g} \times U$, as a simple manipulation demonstrates. Such a $Y^{\prime}$ must be unique, as stated in the following lemma.

Lemma 13.3. Let $j^{*}: Y^{*} \rightarrow Z \times U$ and $j^{\prime}: Y^{\prime} \rightarrow Z \times U$ be closed subschemes which are invariant under $(\Phi \times U)_{/ g \times \operatorname{id}_{U}}$ and $\Phi \times U$-equivalent. Let $\bar{u}: U \rightarrow S \times U$ be a $U$-morphism and assume that $\left(g \times_{U}\right) \circ j^{*}$ and $\left(g \times \operatorname{id}_{U}\right) \circ j^{\prime}$ factor through $\bar{u}: U \rightarrow S \times U$. (That is, they are both on the same "fibre" above $S \times U$.) Then $Y^{*}=Y^{\prime}$.

Proof. To ease the notation, we write $(\bullet)_{U}=\bullet \times U$. We want to prove that the two subfunctors $Y^{*}$ and $Y^{\prime}$ are identical. Let $t^{\prime}: T \rightarrow Y^{\prime} \in \operatorname{Hom}_{U}\left(T, Y^{\prime}\right)$. Since $Y^{*}$ and $Y^{\prime}$ are $\Phi_{U^{-}}$-equivalent, there exists a covering $c: \widetilde{T} \rightarrow T$ together with $\tilde{t}^{*} \in \operatorname{Hom}_{U}\left(\widetilde{T}, Y^{*}\right)$ such that $j^{\prime} t^{\prime} c \equiv j^{*} \tilde{t}^{*} \bmod \Phi_{U}$. Using the commutative diagrams (unnamed arrows are the canonical ones)
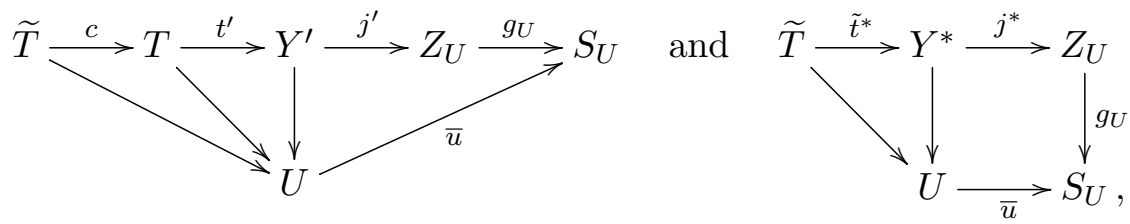

it follows that $j^{\prime} t^{\prime} c \equiv j^{*} \tilde{t}^{*} \bmod \left(\Phi_{U}\right)_{/ g_{U}}$. Since $Y^{*}$ is invariant under $\left(\Phi_{U}\right)_{/ g_{U}}$, we have $j^{\prime} t^{\prime} c=$ $j^{*} \tilde{t}_{1}^{*}$ for some $\tilde{t}_{1}^{*} \in \operatorname{Hom}_{U}\left(\widetilde{T}, Y^{*}\right)$. By descent, $\tilde{t}_{1}^{*}=t_{1}^{*} c$ with $t_{1}^{*} \in \operatorname{Hom}_{U}\left(T, Y^{*}\right)$. We have proved that $j^{\prime}\left(\operatorname{Hom}_{U}\left(T, Y^{\prime}\right)\right) \subseteq j^{*}\left(\operatorname{Hom}_{U}\left(T, Y^{*}\right)\right)$. Applying the same argument with $Y^{*}$ and $Y^{\prime}$ interchanged, we arrive at $Y^{*}=Y^{\prime}$.

The proof of Proposition 13.1 can now be achieved by something more concrete.

Proposition 13.4. Assume $B=U=\operatorname{Spec}(C), S=\operatorname{Spec}(\Lambda), X=\operatorname{Spec}(R)$, and $Z=\operatorname{Spec}(\mathcal{O})$. Assume the existence of etale coordinates $\boldsymbol{x}=\left(x_{1}, \ldots, x_{\ell}\right), \boldsymbol{y}=\left(y_{1}, \ldots, y_{r}\right)$, and $\boldsymbol{z}=\left(z_{1}, \ldots, z_{n}\right)$ for $\Lambda / C, R / \Lambda$, and $\mathcal{O} / R$, respectively. Suppose that the ideal $\mathfrak{A}$ of $\Phi$ in $\widehat{\mathcal{P}}_{\mathcal{O} / C}$ is generated by $n$ elements $F_{1}, \ldots, F_{n}$.

Let $u, u^{\prime}: \Lambda \rightarrow C$ be infinitesimally close $C$-morphisms and write $\mathfrak{u}=\operatorname{Ker}(u)$ and $\mathfrak{u}^{\prime}=\operatorname{Ker}\left(u^{\prime}\right)$. Let $\sigma: \mathcal{O} \rightarrow R / \mathfrak{u} R$ be a $C$-morphism enjoying the following properties:

(a) The morphism $\sigma$ annihilates $\mathfrak{u}$.

(b) The morphism $\sigma$ induces a section of $R / \mathfrak{u} R \rightarrow \mathcal{O} / \mathfrak{u} \mathcal{O}$. 


\section{HOMOTOPY EXACT SEQUENCE}

(c) The closed subscheme $\{\operatorname{Ker}(\sigma)=0\} \subseteq \operatorname{Spec}(\mathcal{O})$ is tangent to the i.e.r. $\Phi_{/ \Lambda}$ rel. $C$.

Then there exists a unique morphism

$$
T\left(\sigma, u^{\prime}\right): \mathcal{O} \longrightarrow R / \mathfrak{u}^{\prime} R
$$

annihilating $\mathfrak{u}^{\prime}$ and inducing a section of $R / \mathfrak{u}^{\prime} R \rightarrow \mathcal{O} / \mathfrak{u}^{\prime} \mathcal{O}$ such that

(i) the subscheme $\left\{\operatorname{Ker}\left(T\left(\sigma, u^{\prime}\right)\right)=0\right\}$ is $\Phi$-equivalent to $\{\operatorname{Ker}(\sigma)=0\}$; and

(ii) the subscheme $\left\{\operatorname{Ker}\left(T\left(\sigma, u^{\prime}\right)\right)=0\right\}$ is tangent to $\Phi_{/ \Lambda}$ rel. $C$.

Let us illustrate the situation using diagrams:
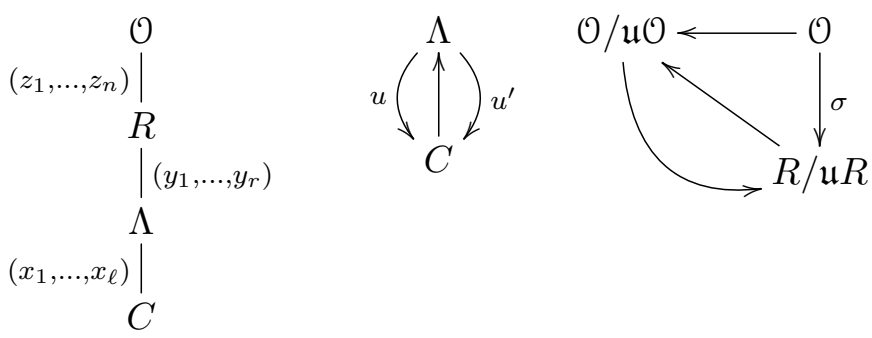

As Lemma 13.3 and the discussion preceding it clarify, the uniqueness of $T\left(\sigma, u^{\prime}\right)$ ensues from properties (i) and (ii). Also, as Lemma 13.2 and the discussion preceding it elucidate, property (ii) is guaranteed by property (i). Finally, the hypothesis made on the number of generators of $\mathfrak{A}$ is not seriously restrictive, as $\widehat{P}_{\Phi} \rightarrow \widehat{P}_{Z / B}$ is a quasi-regular immersion of codimension $n$, due to the total complementarity of $\Phi$ and [EGA, $\mathrm{IV}_{4}$, Proposition 19.1.5, p. 186]. We are then left with the construction of $T\left(\sigma, u^{\prime}\right)$, which is the subject of the following lines.

Write $\boldsymbol{c}=u(\boldsymbol{x})$ and $\boldsymbol{c}^{\prime}=u^{\prime}(\boldsymbol{x})$, so that $\boldsymbol{x}-\boldsymbol{c} \in \mathfrak{u}^{\times \ell}$ and $\boldsymbol{x}-\boldsymbol{c}^{\prime} \in \mathfrak{u}^{\prime \times \ell}$. Fix an arbitrary morphism of noetherian $C$-algebras $\varphi: \mathcal{O} \rightarrow A$ which factors through $\sigma$, and put $\varphi=\bar{\varphi} \circ \sigma$. Note that $\varphi(\boldsymbol{x})=\boldsymbol{c} \cdot 1_{A}$. Define

$$
C[\boldsymbol{x}, \boldsymbol{y}, \boldsymbol{z}] \longrightarrow A, \quad\left\{\begin{aligned}
\boldsymbol{x} & \longmapsto \boldsymbol{c}^{\prime} \\
\boldsymbol{y} & \longmapsto \varphi(\boldsymbol{y}) \\
\boldsymbol{z} & \longmapsto \varphi(\boldsymbol{z})+\boldsymbol{\alpha},
\end{aligned}\right.
$$

where $\boldsymbol{\alpha} \in A^{\times n}$ is nilpotent and will be adjusted later. Since $C[\boldsymbol{x}, \boldsymbol{y}, \boldsymbol{z}] \rightarrow \mathcal{O}$ is etale, we obtain a commutative diagram

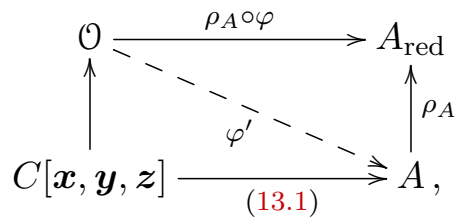

where $\rho_{A}: A \rightarrow A_{\text {red }}$ is the obvious map.

Lemma 13.5. The morphism $\varphi^{\prime}$ constructed above enjoys the following properties:

(i) The morphisms $\varphi$ and $\varphi^{\prime}$ are infinitesimally close.

(ii) $\varphi^{\prime}(\boldsymbol{x})=\boldsymbol{c}^{\prime} \cdot 1_{A}$.

(iii) For each $\lambda \in \Lambda$, we have $\varphi^{\prime}(\lambda)=u^{\prime}(\lambda) \cdot 1_{A}$.

Proof. Only statement (iii) requires justification. The desired conclusion will be reached once we have shown $\rho_{A} \circ \varphi^{\prime}(\lambda)=u^{\prime}(\lambda) \cdot 1_{A_{\text {red }}}$ for all $\lambda \in \Lambda$, in other words, that $\varphi^{\prime}: \Lambda \rightarrow A$ and $u^{\prime}: \Lambda \rightarrow A$ 


\section{J. P. DOs SANTOS}

are infinitesimally close. For $\varphi^{\prime}$ and $u^{\prime}$, send $\boldsymbol{x}$ to $\boldsymbol{c}^{\prime}$, so that the aforementioned equality leads to a commutative diagram

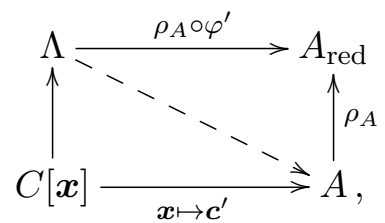

where the dotted arrow can be either $\varphi^{\prime}$ or $u^{\prime}$; as $C[\boldsymbol{x}] \rightarrow \Lambda$ is etale, we arrive at the sought conclusion.

Now,

$$
\begin{array}{rlrl}
\rho_{A}\left\{\varphi^{\prime}(\lambda)\right\} & =\rho_{A}\{\varphi(\lambda)\} & & \left(\text { definition of } \varphi^{\prime}\right) \\
& =\rho_{A}\{\bar{\varphi} \circ \sigma(\lambda)\} & & (\text { definition of } \varphi) \\
& =\rho_{A}\{\bar{\varphi}(u(\lambda))\} & & (\lambda-u(\lambda) \in \mathfrak{u} \mathcal{O} \subseteq \operatorname{Ker}(\sigma)) \\
& =\rho_{A}\left\{\bar{\varphi}\left(u^{\prime}(\lambda)\right)\right\} & & \left(u^{\prime}(\lambda)-u(\lambda)\right. \text { is nilpotent) } \\
& =\rho_{A}\left\{u^{\prime}(\lambda) \cdot 1_{A}\right\} & (\bar{\varphi} \text { is } C \text {-linear }) \\
& =u^{\prime}(\lambda) \cdot 1_{A_{\text {red }}} . &
\end{array}
$$

Corollary 13.6. The ideal $\mathfrak{u}^{\prime} \mathcal{O}$ is annihilated by $\varphi^{\prime}$.

Let us now assume

$$
\varphi: \mathcal{O} \longrightarrow A=\sigma: \mathcal{O} \longrightarrow R / \mathfrak{u} R
$$

Accordingly,

$$
\sigma^{\prime}: \mathcal{O} \longrightarrow R / \mathfrak{u} R=\varphi^{\prime}: \mathcal{O} \longrightarrow A \text {. }
$$

We remind the reader that the definition of $\sigma^{\prime}$ depends upon a parameter $\boldsymbol{\alpha} \in \operatorname{Nil}(R / \mathfrak{u} R)^{\times n}$ which we will have the liberty to adjust further down.

Lemma 13.7. The restriction of $\sigma^{\prime}$ to $R$ induces an isomorphism of $C$-algebras $R / \mathfrak{u}^{\prime} R \rightarrow R / \mathfrak{u} R$.

Proof. Contrary to $\left.\sigma\right|_{R}$, the restriction $\left.\sigma^{\prime}\right|_{R}: R \rightarrow R / \mathfrak{u} R$ does not need to be $R$-linear. But it is certainly $C$-linear and satisfies

$$
\begin{aligned}
\rho_{R / \mathfrak{u} R} \circ \sigma^{\prime}(r) & =\rho_{R / \mathfrak{u} R} \circ \sigma(r) & & \left(\text { construction of } \sigma^{\prime}\right) \\
& =r \cdot 1_{(R / \mathfrak{u} R)_{\mathrm{red}}} & & (\text { definition of } \sigma)
\end{aligned}
$$

for all $r \in R$. In particular, $\sigma^{\prime}: R \rightarrow R / \mathfrak{u} R$ is surjective modulo $\operatorname{Nil}(R / \mathfrak{u} R)$. As $C \rightarrow R / R \mathfrak{u}$ is smooth, it follows that $\operatorname{Nil}(C) \cdot(R / \mathfrak{u} R)=\operatorname{Nil}(R / \mathfrak{u} R)$, from which we deduce that

$$
R / \mathfrak{u}^{\prime} R \stackrel{\sigma^{\prime}}{\longrightarrow} R / \mathfrak{u} R \longrightarrow(R / \mathfrak{u} R) \otimes_{C} C / \operatorname{Nil}(C)
$$

is surjective. We now show that $\sigma^{\prime}: R / \mathfrak{u}^{\prime} R \rightarrow R / \mathfrak{u} R$ is an isomorphism when tensored over $C$ with $C / \operatorname{Nil}(C)$. This will show that $\sigma^{\prime}$ is an isomorphism by a well-known result [Sch68, Lemma 3.3, p. 216]. (We were unable to find a more convenient reference.) Let $r \in R$. If $\sigma^{\prime}(r)$ belongs to

$$
\operatorname{Nil}(C) \cdot(R / \mathfrak{u} R)=\operatorname{Nil}(R / \mathfrak{u} R),
$$

then $\rho_{R / \mathfrak{u} R}\left(\sigma^{\prime}(r)\right)=0$, which gives $r \in \sqrt{\mathfrak{u} R}$. As $u$ and $u^{\prime}$ are infinitesimally close, we have $\sqrt{\mathfrak{u}}=\sqrt{\mathfrak{u}^{\prime}}$, which entails $\sqrt{\mathfrak{u} R}=\sqrt{\mathfrak{u}^{\prime} R}$ (due to smoothness of $\Lambda \rightarrow R$ ). Thus, $r \in \sqrt{\mathfrak{u}^{\prime} R}$, so that $r+\mathfrak{u}^{\prime} R$ is an element of $\operatorname{Nil}\left(R / \mathfrak{u}^{\prime} R\right)$, which equals $\operatorname{Nil}(C) \cdot\left(R / \mathfrak{u}^{\prime} R\right)$. We are done. 
Write $\boldsymbol{F}=\left(F_{1}, \ldots, F_{n}\right)$ and interpret the $F_{i}$ as elements in $\mathcal{O} \llbracket \boldsymbol{d} \boldsymbol{x}, \boldsymbol{d} \boldsymbol{y}, \boldsymbol{d} \boldsymbol{z} \rrbracket=\widehat{\mathcal{P}}_{\mathcal{O} / C}($ see $(8.7)$ and the references nearby). Let $\boldsymbol{F}^{\sigma}$ be the list obtained by mapping each $F_{i}$ to its image in $R / \mathfrak{u} R \llbracket \boldsymbol{d} \boldsymbol{x}, \boldsymbol{d} \boldsymbol{y}, \boldsymbol{d} \boldsymbol{z} \rrbracket$. Clearly, the following holds.

Lemma 13.8. The $R / \mathfrak{u} R$-points of $\operatorname{Spec}(\mathcal{O})$ defined by $\sigma$ and $\sigma^{\prime}$ are $\Phi$-close if and only if

$$
\boldsymbol{F}^{\sigma}\left(\boldsymbol{c}^{\prime}-\boldsymbol{c}, \mathbf{0}, \boldsymbol{\alpha}\right)=\mathbf{0},
$$

where $\boldsymbol{\alpha} \in \operatorname{Nil}(R / \mathfrak{u} R)^{\times n}$ is the parameter introduced in (13.1).

Lemma 13.9. There exists a unique $\boldsymbol{\alpha} \in \operatorname{Nil}(R / R \mathfrak{u})^{\times n}$ satisfying (13.3).

Proof. This is a consequence of Hensel's lemma. Write $\boldsymbol{F}(\boldsymbol{d} \boldsymbol{x}, \mathbf{0}, \boldsymbol{d} \boldsymbol{z})=\sum_{i, j} \boldsymbol{F}_{i j} \cdot \boldsymbol{d} \boldsymbol{x}^{i} \boldsymbol{d} \boldsymbol{z}^{j}$. Fix a positive integer $\mu$ such that $\operatorname{Nil}(R / R \mathfrak{u})^{\mu+1}=0$, and define

$$
\boldsymbol{G}:=\sum_{|i|,|j| \leqslant \mu} \sigma\left(\boldsymbol{F}_{i j}\right)\left(\boldsymbol{c}^{\prime}-\boldsymbol{c}\right)^{i} \cdot \boldsymbol{W}^{j} \in R / R \mathfrak{u}\left[W_{1}, \ldots, W_{n}\right]^{\times n} .
$$

Clearly, for each $\boldsymbol{w} \in \operatorname{Nil}(R / R \mathfrak{u})^{\times n}$ we have $\boldsymbol{F}^{\sigma}\left(\boldsymbol{c}^{\prime}-\boldsymbol{c}, \mathbf{0}, \boldsymbol{w}\right)=\boldsymbol{G}(\boldsymbol{w})$. As the i.e.r. $\Phi$ is complementary to $\operatorname{Spec}(\mathcal{O}) \rightarrow \operatorname{Spec}(R)$, the restriction $\Phi_{/ R}$ is the trivial i.e.r., so

$$
(d x, d y, d z)=(d x, d y, F) .
$$

Regarding $\boldsymbol{c}^{\prime}-\boldsymbol{c}$ and $\boldsymbol{G}$ as line vectors, we then have

$$
W_{i} \equiv\left(\boldsymbol{c}^{\prime}-\boldsymbol{c}\right) \cdot \boldsymbol{L}_{i}+\boldsymbol{G} \cdot \boldsymbol{M}_{i} \bmod \left(W_{1}, \ldots, W_{n}\right)^{\mu+1}, \quad 1 \leqslant i \leqslant n,
$$

where $\boldsymbol{L}_{i}$, respectively $\boldsymbol{M}_{i}$, is an $\ell \times 1$, respectively $n \times 1$, matrix with coefficients in $R / \mathfrak{u} R[\boldsymbol{W}]$. Due to the fact that $\boldsymbol{F}_{00}=\mathbf{0}$, we have

$$
\boldsymbol{G}(\mathbf{0})=\sum_{0<|i| \leqslant \mu} \sigma\left(\boldsymbol{F}_{i j}\right) \cdot\left(\boldsymbol{c}^{\prime}-\boldsymbol{c}\right)^{i} \in \operatorname{Nil}(R / R \mathfrak{u})^{\times n} .
$$

Taking partial derivatives in (13.4) and making $\boldsymbol{W}=\mathbf{0}$, we obtain

$$
\frac{\partial \boldsymbol{G}}{\partial W_{j}}(\mathbf{0}) \cdot \boldsymbol{M}_{i}(\mathbf{0})=\delta_{i j}-\underbrace{\left(\boldsymbol{c}^{\prime}-\boldsymbol{c}\right) \cdot \frac{\partial \boldsymbol{L}_{i}}{\partial W_{j}}(\mathbf{0})}_{\text {nilpotent in } R / \mathfrak{u} R}-\underbrace{\boldsymbol{G}(\mathbf{0}) \cdot \frac{\partial \boldsymbol{M}_{i}}{\partial W_{j}}(\mathbf{0})}_{\text {nilpotent in } R / \mathfrak{u} R} .
$$

Consequently,

$$
{ }^{t}(\operatorname{Jac}(\boldsymbol{G})(\mathbf{0})) \cdot \boldsymbol{M}_{i}(\mathbf{0})=\left[\begin{array}{c}
\delta_{i 1} \\
\vdots \\
\delta_{\text {in }}
\end{array}\right]+\text { nilpotents }
$$

which forces $\operatorname{Jac}(\boldsymbol{G})(\mathbf{0})$ to be in $\mathrm{GL}_{n}(R / \mathfrak{u} R)$. By Hensel's lemma [BouAC, III.4.5, Corollaire 2, p. 270], there exists a unique $\boldsymbol{\alpha} \in \operatorname{Nil}(R / \mathfrak{u} R)^{\times n}$ such that $\boldsymbol{G}(\boldsymbol{\alpha})=\mathbf{0}$, which gives $\boldsymbol{F}^{\sigma}\left(\boldsymbol{c}^{\prime}-\right.$ $\boldsymbol{c}, \mathbf{0}, \boldsymbol{\alpha})=\mathbf{0}$, and we are done.

Now, let $\sigma^{\prime}: \mathcal{O} \rightarrow R / \mathfrak{u} R$ be the morphism defined by means of the element $\boldsymbol{\alpha} \in \operatorname{Nil}(R / \mathfrak{u} R)^{\times n}$ obtained in Lemma 13.9. Since the $R / \mathfrak{u} R$-points of $\operatorname{Spec}(\mathcal{O})$ defined by $\sigma$ and $\sigma^{\prime}$ are $\Phi$-close, it follows that the closed subschemes $\{\operatorname{Ker}(\sigma)=0\}$ and $\left\{\operatorname{Ker}\left(\sigma^{\prime}\right)=0\right\}$ are $\Phi$-equivalent. Let

$$
T\left(\sigma, u^{\prime}\right):=\left(\text { inverse of isomorphism in Lemma 13.7) } \circ \sigma^{\prime}\right. \text {. }
$$

Then $\operatorname{Ker}\left(T\left(\sigma, u^{\prime}\right)\right)=\operatorname{Ker}\left(\sigma^{\prime}\right)$, so that $\left\{\operatorname{Ker}\left(T\left(\sigma, u^{\prime}\right)\right)=0\right\}$ and $\{\operatorname{Ker}(\sigma)=0\}$ are $\Phi$-equivalent. Furthermore, since $\sigma^{\prime}$ annihilates $\mathfrak{u}^{\prime} \mathcal{O}$, the very definition of the isomorphism in Lemma 13.7 shows that $T\left(\sigma, u^{\prime}\right)$ is a section of $R / \mathfrak{u}^{\prime} R \rightarrow \mathcal{O} / \mathfrak{u}^{\prime} \mathcal{O}$. 


\section{J.P. DOS SAntos}

We have therefore proved the existence of the morphism $T\left(\sigma, u^{\prime}\right)$ complying with the two conditions mentioned in Proposition 13.4. (Recall that the tangency property follows from the other properties, as explained in the lines following Proposition 13.1.) With this, we have concluded the proof of Proposition 13.4, and arrive at our goal.

Corollary 13.10. Assume in addition that $f: X \rightarrow S$, respectively $g: Z \rightarrow S$, is proper and geometrically connected, respectively quasi-projective. Let $\pi: H_{f}(Z, \Phi) \rightarrow S$ be the $S$-scheme of Definition 10.4 and endow it with the i.e.r. found in Definition 11.3. Then $H_{\Phi}$ is totally complementary (Definition 7.10 and following lines) to $\pi: H_{f}(Z, \Phi) \rightarrow S$.

\section{Description of $H_{f}(Z, \Phi)$ in the particular case where $S=\operatorname{Spec} k$ and $Z$ stems from the fundamental group scheme}

Let $k$ be an algebraically closed field. The category $\mathbf{S c h}$ is the category of all noetherian separated $k$-schemes and in this section, all schemes and morphisms are to be taken in Sch. Let $X \in \mathbf{S c h}$ be of finite type.

\subsection{Simple stratified schemes}

Let

$$
\left\{\alpha_{\mu}: P_{X}^{\mu} \times_{X} E \longrightarrow E: \mu \in \mathbb{N}\right\}
$$

define a $k$-linear stratification of the $X$-scheme $\theta: E \rightarrow X$ (see Section 5). Write $\alpha$ for the morphism of formal schemes $\widehat{P}_{X} \times_{X} E \rightarrow E$ induced by the system $\left(\alpha_{\mu}\right)$.

Recall that a closed subscheme $Y \subseteq E$ is invariant or saturated if $\alpha$ sends $Y \times_{X} \widehat{P}_{X}$ into $Y$.

Definition 14.1. With the above notation, we say that $E$ is a simple stratified $X$-scheme if the only non-empty invariant closed subscheme of $E$ is $E$ itself.

If $\theta$ is affine and $X$ is smooth, then invariance of $Y$ means invariance of the ideal $\mathcal{J}_{Y} \subseteq \mathcal{O}_{E}$ by $\mathcal{D}_{X / k}$. We also note that if $\alpha$ is simple, then $E$ must be connected, since $|\alpha|$ is just the identity on the underlying topological spaces.

Proposition 14.2 (Universal simplicity). Let $X$ be smooth and irreducible, and let $\theta$ be affine. Suppose that the stratification $\alpha$ is simple. Fix a scheme of finite type $T$, and let $j: Y \rightarrow E \times T$ be a non-empty closed subscheme. Assume that

(i) the morphism $\alpha_{\mu} \times \operatorname{id}_{T}:\left(E \times_{X} P_{X}^{\mu}\right) \times T \rightarrow E \times T$ preserves $Y$ for each $\mu$; and

(ii) the morphism $Y \rightarrow T$ has a section $\sigma: T \rightarrow Y$.

Then $Y=E \times T$.

Proof. If $U \rightarrow T$ is a morphism of schemes, then $Y \times_{T} U \subseteq E \times U$ is also invariant and $Y \times_{T} U \rightarrow U$ has a section. By Lemma 14.3 below, it is therefore sufficient to treat the case where $T=\operatorname{Spec} A$ is the spectrum of a local Artin $k$-algebra. We proceed by induction on $\operatorname{dim}_{k} A$ to show that $j$ is an isomorphism. If $\operatorname{dim}_{k} A=1$, there is nothing to be proved. Let $\varepsilon \in \mathfrak{r}(A) \backslash\{0\}$ be such that $\varepsilon \cdot \mathfrak{r}(A)=0$ and denote by $A_{1}$ the quotient $A / \varepsilon A$. Now $Y \otimes_{A} A_{1} \subseteq E \otimes_{k} A_{1}$ is invariant and hence must equal $E \otimes_{k} A_{1}$. Let $\mathcal{J} \subseteq \mathcal{O}_{E} \otimes_{k} A$ be the ideal sheaf of $Y$; since $Y \otimes_{A} A_{1}=E \otimes_{k} A_{1}$, it follows that $\mathcal{J} \subseteq \mathcal{O}_{E} \otimes_{k} \varepsilon A$. Define a sheaf of ideals of $\mathcal{O}_{E}$ by

$$
\mathcal{J}^{\prime}:=\left\{\psi \in \mathcal{O}_{E}:(\psi \otimes 1) \cdot(1 \otimes \varepsilon) \in \mathcal{J}\right\} .
$$




\section{HOMOTOPY EXACT SEQUENCE}

This is clearly coherent (as a kernel between a morphism of coherent $\mathcal{O}_{E}$-modules). Because $\mathcal{P}_{X}^{\mu}$ is locally free, it is easy to verify that the closed subscheme of $E$ cut out by $\mathcal{J}^{\prime}$ is invariant and, therefore, $\mathcal{J}^{\prime}$ is either $\mathcal{O}_{E}$ or $(0)$. Since $\mathcal{J} \neq(0)$ and $\varepsilon A \simeq k$, it is not hard to see that $\mathcal{J}^{\prime} \neq(0)$. Thus, 1 is a global section of $\mathcal{J}^{\prime}$, so $1 \otimes \varepsilon$ is a global section of $\mathcal{J}$. This gives a contradiction with the fact that $Y \rightarrow T$ is dominant.

Lemma 14.3. Let $f: M \rightarrow T$ be a morphism of algebraic $k$-schemes, and let $j: N \hookrightarrow M$ be a closed subscheme. Then $j$ is an isomorphism if for each spectrum $D$ of a local Artin $k$-algebra of residue field $k$ and each morphism $\tau: D \rightarrow T$, the closed embedding $j \times_{T} \mathrm{id}_{D}$ is an isomorphism.

An application of Proposition 14.2 which will be useful later is as follows.

Lemma 14.4. Let $X$ be smooth and connected. Denote by $\mathfrak{U}_{X}$ the universal torsor and by $\mathfrak{U}_{X}[\bullet]$ the functor

$$
\left\{\begin{array}{c}
\text { actions } \rho \text { of } \Pi(X) \text { on the left of an algebraic } \\
\text { scheme } F \text { such that } \mathcal{U}_{X} \times^{\rho} F \text { is a scheme }
\end{array}\right\} \longrightarrow\{\text { stratified } X \text {-schemes }\} .
$$

Then $\mathcal{U}_{X}[\bullet]$ is fully faithful.

Proof. This is easy, but tedious. Denote by $\mathcal{S}_{X}$ the domain of $\mathfrak{U}_{X}[\bullet]$. For each $F \in \mathcal{S}_{X}$, let $\chi_{F}$ denote the natural projection from $\mathcal{U}_{X} \times F$ to the quotient $\mathcal{U}_{X}[F]$. We know [Jan87, Part 1, 5.14, p. 88] that

$$
\left(\mathrm{pr}, \chi_{F}\right): \mathfrak{U}_{X} \times F \longrightarrow \mathcal{U}_{X} \times_{X} \mathcal{U}_{X}[F]
$$

is an isomorphism of $\mathcal{U}_{X}$-schemes. In particular, $\mathcal{U}_{X}[\bullet]$ is faithful. Endowing $\mathcal{U}_{X} \times F$ with its standard action of $\Pi(X)$ and $\mathfrak{U}_{X} \times_{X} \mathfrak{U}_{X}[F]$ with the action on the first factor only, it follows that (14.1) is $\Pi(X)$-equivariant. Furthermore, endowing $\mathcal{U}_{X} \times_{X} \mathcal{U}_{X}[F]$ with the product stratification, it follows that $\left(\mathrm{pr}, \chi_{F}\right)$ is an isomorphism of stratified $X$-schemes.

We now deal with fullness. We take $F, F^{\prime} \in \mathcal{S}_{X}$ (recall that they are separated), and let $\varphi: \mathcal{U}_{X}[F] \rightarrow \mathcal{U}_{X}\left[F^{\prime}\right]$ be a morphism of stratified $X$-schemes. Using (14.1), we obtain a morphism of $\mathcal{U}_{X}$-schemes

$$
\bar{\varphi}=(\operatorname{pr}, \theta): \mathcal{U}_{X} \times F \longrightarrow \mathcal{U}_{X} \times F^{\prime} .
$$

It is not hard to see that $\bar{\varphi}$ respects the usual stratifications.

Recall that $\mathcal{U}_{X}$ is a projective limit of stratified torsors:

$$
\mathcal{U}_{X}=\underset{\lambda}{\lim } \mathcal{U}_{\lambda}
$$

where each $\mathcal{U}_{\lambda} \rightarrow X$ is a torsor under an algebraic group scheme. In fact, $\mathcal{U}_{\lambda}$ corresponds to some affine quotient $\Pi(X) \rightarrow \Pi_{\lambda}$, so that any closed non-empty subscheme of $\mathcal{U}_{\lambda}$ which is invariant under the stratification corresponds to a closed subgroup scheme of $\Pi_{\lambda}$ which is invariant under $\Pi(X)$; consequently, the stratification of $\mathcal{U}_{\lambda}$ is simple.

From [EGA, $\mathrm{IV}_{3}$, Théorème 8.8.2, p. 28] there exist an index $\lambda$ and a morphism of $\mathcal{U}_{\lambda \text {-schemes }}$

$$
\bar{\varphi}_{\lambda}=\left(\operatorname{pr}, \theta_{\lambda}\right): \mathcal{U}_{\lambda} \times F \longrightarrow \mathcal{U}_{\lambda} \times F^{\prime}
$$

such that

$$
\bar{\varphi}_{\lambda} \times{u_{\lambda}}_{\lambda} \operatorname{id}_{\mathfrak{u}_{X}}=\bar{\varphi}
$$




\section{J. P. DOs SANTOS}

In particular, we have a commutative diagram

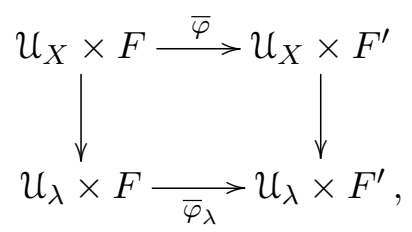

where the vertical arrows respect stratifications and are faithfully flat and affine morphisms. Using this diagram, one is able to verify that $\bar{\varphi}_{\lambda}$ is a morphism of stratified $X$-schemes.

Let $u_{0}$ be a $k$-point of $\mathcal{U}_{\lambda}$ above $x_{0}$, and let $Y$ be the closed subscheme of $\mathcal{U}_{\lambda} \times F$ where $\theta_{\lambda}$ coincides with $(u, y) \mapsto \theta_{\lambda}\left(u_{0}, y\right)$ (recall that all schemes we consider here are separated). Since $\bar{\varphi}_{\lambda}$ is a morphism of stratified schemes, it follows that $Y$ fulfils conditions (i) and (ii) of Proposition 14.2 , so that $Y=\mathcal{U}_{\lambda} \times F$. Let $a: F \rightarrow F^{\prime}$ be the morphism $y \mapsto \theta_{\lambda}\left(u_{0}, y\right)$; we have just proved that $\bar{\varphi}_{\lambda}=\operatorname{id}_{\mathcal{U}_{\lambda}} \times a$. It is now easily verified that $a$ induces $\varphi$.

\subsection{Determination of $H(Z, \Phi)$}

Let us take $X$ smooth and projective. We give ourselves a projective scheme $F$, an algebraic group $G$, a principal $G$-bundle $\theta: E \rightarrow X$, and an action $G \rightarrow \mathbf{A u t}_{k}(F)$. Moreover, we assume that $E$ is a stratified principal $G$-bundle and let $\Gamma$ be the accompanying i.e.r. Note that, in this case, the equivalence classes of $\Gamma$ are preserved by the action of $G$. Last, we take for granted that the source of the associated fibration [Jan87, $\S 5$, Part I]

$$
\zeta: E \times{ }^{G} F \longrightarrow X
$$

is represented by a quasi-projective $X$-scheme $Z$. We will denote $(\Gamma \times F) / G$, the induced i.e.r. in $Z$, by $\Phi$.

In the following proposition, $F^{G}$ is the scheme defined in [Jan87, Part I, 2.6, p. 27ff].

Proposition 14.5. Assume that the stratification on $E$ is simple. Then $H(Z, \Phi)$ is isomorphic to $F^{G}$.

Before proceeding to the proof, we describe how such a $Z$ should come about. Let $G$ be a quotient of the fundamental stratified group scheme $\Pi(X)$ (see $\S 6.1$ ) of $X$ at some $k$-rational point of $X$. We define $E$ as being the stratified torsor associated with $G$, that is, $E=\mathcal{U}_{X} \times{ }^{\Pi(X)} G$; see again $\S 6.1$. (This also equals the quotient of $\mathcal{U}_{X}$ by the kernel of $\Pi(X) \rightarrow G$.) It is not hard to note that this stratification is simple, as an invariant closed subscheme gives rise to an invariant ideal of $\mathcal{O}_{E}$; see also the justification of simplicity offered for " $\mathfrak{U}_{\lambda}$ " (lines following (14.2)). We then choose a representation $\rho: G \rightarrow \mathbf{P G L}_{m}$ leaving invariant a subscheme $F$ of $\mathbb{P}^{m-1}$ and projective descent [BLR90, $\S 6.1$, Theorem 7, p. 138] ensures that $Z=E \times^{G} F$ is a scheme.

Proof of Proposition 14.5. We start by defining a natural transformation

$$
\omega: F^{G} \longrightarrow H(Z, \Phi) \text {. }
$$

For this, in all that follows, we let $G$ act on the right of $E \times F$ in the standard fashion, namely $(p, y) \cdot g=\left(p g, g^{-1} y\right)$, and trivially on parameter schemes $T$.

Given a parameter scheme $T \in \mathbf{S c h}$ and $a: T \rightarrow F^{G}$, we note that $\operatorname{id}_{E} \times a: E \times T \rightarrow E \times F$ is $G$-equivariant and interweaves $\Gamma \times T$ and $\Gamma \times F$. Consequently, using that quotients commute with products [DG70, III.1.1.8, p. 287], we can define a morphism of $X$-schemes

$$
\omega_{T}(a): X \times T \longrightarrow Z
$$




\section{HOMOTOPY EXACT SEQUENCE}

interweaving $\Gamma / G \times T$, which is just $X_{\infty} \times T$ as $\Gamma$ is totally complementary to $\theta: E \rightarrow X$, and $\Phi=(\Gamma \times F) / G$. Clearly, $\omega_{T}$ respects compositions, and we have constructed the natural transformation $\omega$.

If $\chi: E \times F \rightarrow Z$ denotes the quotient morphism, the arrow

$$
\left(\mathrm{pr}_{E}, \chi\right): E \times F \longrightarrow E \times_{X} Z
$$

is a G-equivariant isomorphism [Jan87, Part I, 5.14, p. 88] of E-schemes, which results from the fact that $a: T \rightarrow F^{G}$ can be reobtained from $\omega(a)$. Hence, $\omega_{T}: F^{G}(T) \rightarrow H(Z, \Phi)(T)$ is always injective.

We now aim at showing that $\omega_{T}$ is surjective. Let $T \in \mathbf{S c h}$ and let

$$
\sigma: X \times T \longrightarrow Z
$$

be a morphism of $X$-schemes (a family of sections of $\zeta$ ). By means of $\sigma$ and the isomorphism in (14.4), we derive a $G$-equivariant morphism

$$
h_{\sigma}: E \times T \longrightarrow F
$$

rendering

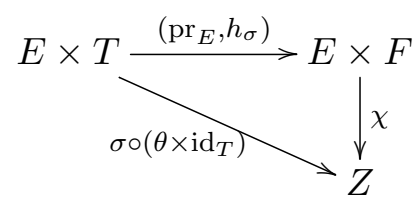

commutative. At this point we wish to show that $h_{\sigma}$ is "constant on certain equivalence classes of $\Gamma \times T^{\prime \prime}$.

Lemma 14.6. Fix $U \in \mathbf{S c h}$ and let $\left(p_{0}, t\right),\left(p_{1}, t\right) \in E(U) \times T(U)$ be $\Gamma \times T$-equivalent. Assume that the $U$-points

$$
\chi\left(p_{0}, h_{\sigma}\left(p_{0}, t\right)\right) \text { and } \chi\left(p_{1}, h_{\sigma}\left(p_{1}, t\right)\right)
$$

of $Z$ are $\Phi$-equivalent. Then $h_{\sigma}\left(p_{0}, t\right)=h_{\sigma}\left(p_{1}, t\right)$.

Proof. Let us write $h=h_{\sigma}$ to simplify. The fact that $\chi\left(p_{0}, h\left(p_{0}, t\right)\right)$ and $\chi\left(p_{1}, h\left(p_{1}, t\right)\right)$ are equivalent modulo $\Phi$ means that we can find an fppf covering $c: U^{\prime} \rightarrow U$ and an element $g^{\prime} \in G\left(U^{\prime}\right)$ such that

$$
\left(p_{0} c, h\left(p_{0}, t\right) c\right) \cdot g^{\prime} \equiv\left(p_{1} c, h\left(p_{1}, t\right) c\right) \quad \bmod \Gamma \times F .
$$

Hence, $\left(p_{0} c\right) \cdot g^{\prime} \equiv_{\Gamma} p_{1} c$; since $p_{0} \equiv_{\Gamma} p_{1}$, we obtain

$$
\left(p_{1} c\right) \cdot g^{\prime} \equiv_{\Gamma}\left(p_{0} c\right) \cdot g^{\prime} \equiv_{\Gamma} p_{1} c .
$$

Since $\Gamma$ is complementary to $\theta: E \rightarrow X$ (Definition 7.9) and $\theta\left(p_{1} c\right)=\theta\left(p_{1} c \cdot g^{\prime}\right)$, we arrive at $\left(p_{1} c\right) \cdot g^{\prime}=p_{1} c$, which gives $g^{\prime}=\mathrm{id}$. Therefore, $h\left(p_{0}, t\right) c=h\left(p_{1}, t\right) c$ and hence $h\left(p_{0}, t\right)=h\left(p_{1}, t\right)$ by descent.

We now assume that $T$ is algebraic and that $\sigma$ interweaves $X_{\infty} \times T$ and $\Phi$, so that $\sigma$ corresponds to a $T$-point of $H(Z, \Phi)$. Let $p_{0}$ : Spec $(k) \rightarrow E$ be a point of $E$; by means of $p_{0}$, define a closed subscheme $Y$ of $E \times T$ having as $U$-points the set

$$
Y(U)=\left\{(p, t) \in E(U) \times T(U): h_{\sigma}(p, t)=h_{\sigma}\left(p_{0}, t\right)\right\} .
$$

This $Y$ enjoys the following properties: 


\section{J. P. DOS SANTOS}

(a) The scheme $Y$ is invariant under $\Gamma \times T$ due to the interweaving property of $\sigma$, diagram (14.5), and Lemma 14.6.

(b) The morphism $\left(p_{0}, \mathrm{id}_{T}\right): T \rightarrow Y$ is a section of $\operatorname{pr}_{T}: Y \rightarrow T$.

As the stratification on $E$ is simple, Proposition 14.2 lets us conclude that $Y=E \times T$. Hence, for each $U \in \mathbf{S c h}, p \in E(U)$, and $t \in T(U)$, we have $h_{\sigma}(p, t)=h_{\sigma}\left(p_{0}, t\right)$, which means that, writing

$$
a_{\sigma}:=\left.h_{\sigma}\right|_{\left\{p_{0}\right\} \times T},
$$

we have

$$
h_{\sigma}=a_{\sigma} \circ \mathrm{pr}_{T} .
$$

From the fact that $h_{\sigma}$ is $G$-equivariant (recall that $T$ has the trivial $G$-action) we conclude that $a_{\sigma}$ takes its values in $F^{G}$.

We hence conclude that $\sigma=\omega_{T}\left(a_{\sigma}\right)$, which shows that $\omega_{T}$ is surjective for $T \in \mathbf{S c h}$ algebraic. A standard limit argument [EGA, $\mathrm{IV}_{3}$, Théorème 8.8.2, p. 28] then shows that $\omega_{T}$ is unrestrictedly surjective, and the proof of Proposition 14.5 is achieved.

\section{Proof of the main result}

We now prove the main result, Theorem 1.1(ii). The notation and conventions are those of the statement. We rarely mention references to base points from now on.

We wish to deal with some preparatory material on category theory. Our arrows go in the opposite direction of those in [ML98, III.1, Definition, p. 55].

Definition 15.1. Let $F: A \rightarrow B$ be a functor, and let $b \in B$. A universal arrow from $F$ to $b$ is a pair $\left(b_{*}, u\right)$ consisting of an object $b_{*}$ of $A$ and an arrow $u: F b_{*} \rightarrow b$ such that for any $\alpha: F a \rightarrow b$, there exists a unique $\widetilde{\alpha}: a \rightarrow b_{*}$ rendering commutative the diagram

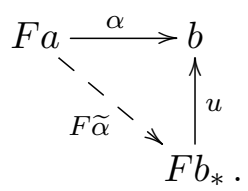

An element $b_{*}$ is universal from $F$ to $b$ if there exists a universal arrow from $F$ to $b$ of the form $(u, b)$.

Another way to define the previous concept is the following [ML98, III.2, p. 59]. If $u: F b_{*} \rightarrow b$ is universal from $F$ to $b$, then

$$
\eta_{\left(u, b_{*}\right)}: \operatorname{Hom}_{A}\left(-, b_{*}\right) \longrightarrow \operatorname{Hom}_{B}(F(-), b), \quad\left(x \stackrel{\beta}{\rightarrow} b_{*}\right) \longmapsto\left(F x \stackrel{F \beta}{\rightarrow} F b_{*} \stackrel{u}{\rightarrow} b\right)
$$

is a natural isomorphism of contravariant functors. Conversely, any natural isomorphism between these two functors is of the form $\eta_{\left(u, b_{*}\right)}$ for a unique universal arrow $u: F b_{*} \rightarrow b$.

LEMMA 15.2. Let

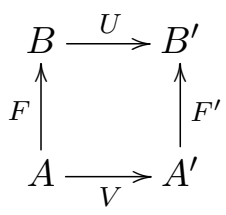




\section{HOMOTOPY EXACT SEQUENCE}

be a commutative diagram between categories (up to natural isomorphism). Assume that $U$ and $V$ are fully faithful. Let $b \in B$ and let $b_{*} \in A$ be such that $V\left(b_{*}\right)$ is universal from $F^{\prime}$ to $U b$. Then $b_{*}$ is universal from $F$ to $b$.

Proof. We have natural isomorphisms

$$
\begin{aligned}
& \operatorname{Hom}_{A}\left(-, b_{*}\right) \stackrel{\sim}{\longrightarrow} \operatorname{Hom}_{A^{\prime}}\left(V(-), V\left(b_{*}\right)\right) \\
& \stackrel{\sim}{\longrightarrow} \operatorname{Hom}_{B^{\prime}}\left(F^{\prime} V(-), U(b)\right) \\
& \stackrel{\sim}{\longrightarrow} \operatorname{Hom}_{B^{\prime}}(U F(-), U(b)) \\
& \stackrel{\sim}{\longrightarrow} \operatorname{Hom}_{B}(F(-), b) \text {. }
\end{aligned}
$$

This lemma will be applied to the categories and functors introduced of $\S 6.2$. Recall from that section that $\mathcal{R}_{X}$ denotes the category of proper schemes $F$ endowed with a left action of $\Pi(X)$ such that $\mathcal{U}_{X} \times{ }^{\Pi(X)} F$ is an $X$-scheme. We then have functors

$$
\mathcal{U}_{X}[\bullet]:=\mathcal{U}_{X} \times{ }^{\Pi(X)}(\bullet) \text { and } \mathcal{U}_{S}[\bullet]:=\mathcal{U}_{S} \times{ }^{\Pi(S)}(\bullet) .
$$

(See $\S 6.2$ for more details concerning them.) Using the isomorphism of stratified presheaves

$$
\left(\mathcal{U}_{X} \times F\right) / \Pi(X) \stackrel{\sim}{\longrightarrow}\left\{\left[\mathcal{U}_{X} \times \Pi(S)\right] / \Pi(X) \times F\right\} / \Pi(S),
$$

the isomorphism of stratified schemes $\mathfrak{U}_{X} \times \Pi(X) \Pi(S) \simeq \mathcal{U}_{S} \times_{S} X$ [Nor76, Proposition 2.9(c)], the isomorphism of stratified presheaves

$$
\left\{\left(\mathcal{U}_{S} \times_{S} X\right) \times F\right\} / \Pi(S) \stackrel{\sim}{\longrightarrow}\left\{\left(\mathcal{U}_{S} \times F\right) / \Pi(S)\right\} \times_{S} X,
$$

and the fact that "taking the associated sheaf commutes with fibre products" [SGA4, II, Proposition 4.3 and I, Définition 2.5], it is possible to verify that the following diagram commutes:

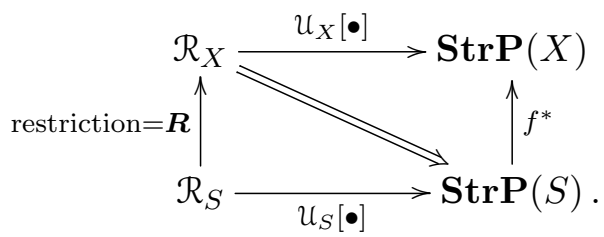

Let $\widetilde{\rho}: \Pi(X) \rightarrow \mathbf{G L}(V)$ be a finite-dimensional representation of $\Pi(X)$, and let $\rho: \Pi(X) \rightarrow$ $\mathbf{P G L}(V)$ be the induced projective representation. Let

$$
\pi: H=H_{f}\left(\mathcal{U}_{X}[\mathbb{P}(V), \rho]\right) \longrightarrow S
$$

denote the $S$-scheme of Definition 10.4. We know from Corollary 13.10 and Theorem 10.5 that $H$ is a stratified scheme over $S$ whose connected components are projective. (There is no reason a priori for $\pi$ to be quasi-compact.) Consequently, any connected component is a stratified proper scheme over $S$ and, as such, is mapped onto $S$; see Lemma 6.2.

We also know that $\pi^{-1}\left(s_{0}\right) \simeq \mathbb{P}(V)^{\operatorname{Im}(a)}$ : apply Corollary 12.4 , the commutativity of the above diagram in the case $X_{0} \rightarrow X$, and Proposition 14.5. As $\mathbb{P}(V)^{\operatorname{Im}(a)}$ has only finitely many connected components, we conclude that $H$ has only finitely many connected components. We have deduced that $\pi$ is proper.

As the automorphism group scheme of $\mathbb{P}(V)^{\operatorname{Im}(a)}$ is affine (see Proposition 16.3), we obtain a homomorphism $\tau: \Pi(S) \rightarrow \mathbf{A u t}_{k}\left(\mathbb{P}(V)^{\operatorname{Im}(a)}\right)$ and an isomorphism $\operatorname{in} \operatorname{Str} \mathbf{S}(S)$

$$
\mathcal{U}_{S}\left[\mathbb{P}(V)^{\operatorname{Im}(a)}, \tau\right] \simeq H .
$$




\section{J. P. DOS SANTOS}

(See Proposition 6.4.) Since $H$ is universal from $f^{*}$ to $\mathcal{U}_{X}[\mathbb{P}(V), \rho]$ (see Corollary 12.3) and both $\mathcal{U}_{S}[\bullet]$ and $\mathcal{U}_{X}[\bullet]$ are fully faithful (Lemma 14.4 ), Lemma 15.2 allows us to say that $\mathbb{P}(V)^{\operatorname{Im}(a)}$, with the aforementioned action of $\Pi(S)$, is universal from $\boldsymbol{R}$ to $(\mathbb{P}(V), \rho)$. Using that $\Pi(X) / \operatorname{Ker}(b) \simeq$ $\Pi(S)$, we deduce that $\mathbb{P}(V)^{\operatorname{Ker}(b)}$ is universal from $\boldsymbol{R}$ to $(\mathbb{P}(V), \rho)$. We conclude that the $\Pi(S)$ schemes $\mathbb{P}(V)^{\operatorname{Im}(a)}$ and $\mathbb{P}(V)^{\operatorname{Ker}(b)}$ are isomorphic. A fortiori, the reduced $k$-schemes underlying $\mathbb{P}(V)^{\operatorname{Ker}(b)}$ and $\mathbb{P}(V)^{\operatorname{Im}(a)}$ are isomorphic, so that the closed embedding

$$
\mathbb{P}(V)^{\operatorname{Ker}(b)} \longrightarrow \mathbb{P}(V)^{\operatorname{Im}(a)}
$$

must induce a bijection on $k$-points. This means that Property 4.2 holds, and we conclude that $\operatorname{Im}(a)=\operatorname{Ker}(b)$ using Lemma 4.3 and then Lemma 4.2 .

Remark 15.3. The cautious reader must have realized

(i) that the fact that $\mathcal{U}_{X}[\mathbb{P}(V), \rho]$ is a scheme and $\mathcal{U}_{X}[\mathbb{P}(V), \rho] \rightarrow X$ is projective and smooth needs justification;

(ii) that $F:=\mathbb{P}(V)^{\operatorname{Ker}(b)}$ is not necessarily an element of $\mathcal{R}_{S}$; for that, we need $\mathcal{U}_{S} \times \Pi(S) F$ to be a scheme. (See (6.2) for the definition.)

In the following lines we justify these claims.

(i) Pick an algebraic quotient $G$ of $\Pi(X)$ such that $\Pi(X) \rightarrow \mathbf{G L}(V)$ factors through $G$. Then

$$
\mathcal{U}_{X} \times{ }^{\Pi(X)} \mathbb{P}(V) \simeq\left(\mathcal{U}_{X} \times{ }^{\Pi(X)} G\right) \times{ }^{G} \mathbb{P}(V) \simeq P \times{ }^{G} \mathbb{P}(V),
$$

and $P \rightarrow X$ is a $G$-torsor. It follows from [BLR90, Theorem 7, p. 138] that $P{ }^{G} \mathbb{P}(V)$ is a scheme and that the obvious morphism to $X$ is quasi-projective. That it is proper, respectively smooth, follows from [EGA, $\mathrm{IV}_{2}$, Proposition 2.7.1, p. 29], respectively [EGA, IV 4 , Corollaire 17.7.3, p. 72].

(ii) Pick an algebraic quotient $G$ of $\Pi(X)$ such that $\Pi(X) \rightarrow \mathbf{G L}(V)$ factors through $G$. Let $K \unlhd \Pi(X)$ be the kernel of $\Pi(X) \rightarrow G$. Let $L$ be the image of $K$ in $\Pi(S)$. As $b$ is a quotient morphism, $L \unlhd \Pi(S)$ and $H:=\Pi(S) / L$ is a quotient of $G$. It is then straightforward to show that $L$ acts trivially on $F$. Hence,

$$
\begin{aligned}
\mathcal{U}_{S} \times{ }^{\Pi(S)} F & \simeq\left(\mathcal{U}_{S} \times{ }^{\Pi(S)} H\right) \times{ }^{H} F \\
& \simeq P \times{ }^{H} F,
\end{aligned}
$$

where $P \rightarrow S$ is an $H$-torsor. (This detour intends simply to avoid analysis of the morphism $\Pi(S) \rightarrow \mathbf{A u t}_{k}(F)$.) As $H$ and $P$ are reduced and $k$ is perfect, $(P \times F)_{\text {red }}=P \times F_{\text {red }}$ is invariant under $H$. We are then in a position to apply [DG70, III.2.7.1, p. 318], which guarantees that $(P \times F) / H$ is representable if $\left(P \times F_{\text {red }}\right) / H$ is likewise. Since $\operatorname{Ker}(b)$ acts on $\mathbb{A}(V)$, the scheme $F_{\text {red }}$ is a disjoint union of projective spaces. Consequently, the action of $H$ on $F_{\text {red }}$ can be linearized by using the anti-canonical bundle [GIT, Chapter 1, $\S 3$; projective fpqc descent [BLR90, 6.1, Theorem 7, p. 138] ensures the representability of $\left(P \times F_{\text {red }}\right) / H$.

\section{Digression on specific automorphism group schemes}

Our goal here is to study a simple instance where automorphism group schemes are affine. These are tailored to fit some technical requisites found in studying the schemes $\mathbb{P}(V)^{G}$ appearing in $\S$ 4.2. The most relevant result here is Proposition 16.3. Indeed, if $G$ is an affine group scheme 


\section{Homotopy EXACT SEQUENCE}

(over $k$ ) and $V$ is a finite-dimensional representation of it, then

$$
\left(\mathbb{P}(V)^{G}\right)_{\text {red }}=\coprod_{\lambda \text { a character of } G} H_{\lambda}
$$

where

$$
H_{\lambda}=\mathbb{P}(\{v \in V: G \text { acts on } v \text { through } \lambda\}) .
$$

Note that, already in the case where $\mathbb{G}_{a}$ acts on $\mathbb{P}^{1}$ by "translations", the fixed-point scheme is not reduced.

Throughout, Sch denotes the category of schemes over an algebraically closed field $k$, where all morphisms and constructions take place. The full subcategory of all reduced schemes is denoted by $\mathbf{R S c h}$.

The reader should bear in mind that for a proper scheme $M$, the functor of its automorphisms

$$
\operatorname{Aut}_{k}(M): \mathbf{S c h}^{o} \longrightarrow \operatorname{Grp}, \quad T \longmapsto \operatorname{Aut}_{T}(M \times T)
$$

is represented by a group scheme which is locally of finite type. For a proof under the extra assumption of projectivity, see [Nit05, Exercise following Theorem 5.23, p. 133]. The more general case is in [MO67, Theorem 3.7, p. 17].

We now present some basic tools employed in this section. First, we state two results which will be much used: Lemma 16.1 and Lemma 16.2. Proofs are in [EGA, I, Corollaire 5.1.10, p. 131] and [SGA3, $\mathrm{VI}_{\mathrm{A}}, 2.4 .1$, p. 304], respectively.

Lemma 16.1. Let $W$ be a scheme of finite type over $k$. Then $W$ is affine if and only if $W_{\text {red }}$ is likewise.

Lemma 16.2. Let $G$ be a group scheme locally of finite type over $k$. If $G$ is connected, then $G$ is of finite type.

Second, we discuss the neutral component of a group scheme. Given a group scheme $G$ locally of finite type over $k$, we let $G^{0}$ denote the connected component of the identity of the topological space underlying $G$. Since $G$ is locally noetherian, $G^{0}$ is an open subset of $G$ [EGA, I, Corollaire 6.1.9]. As $G^{0} \times G^{0}$ is connected, we obtain a group scheme structure on $G^{0}$ which turns the inclusion into a morphism of group schemes. The group scheme $G^{0}$ is called the neutral component of $G$. Due to Lemma $16.2, G^{0}$ is of finite type over $k$. For more on this, the reader should consult [SGA3, $\left.\mathrm{VI}_{\mathrm{A}}, \S 2\right]$.

Proposition 16.3. Let $M$ be projective. Assume that, for each connected component $C$ of $M_{\text {red }}$, $\mathbf{A u t}_{k}(C)$ is affine and its Néron-Severi group

$$
\mathrm{NS}(C)=\operatorname{Pic}(C) / \operatorname{Pic}^{0}(C)
$$

has rank one (as a finitely generated abelian group). Then $\mathbf{A u t}_{k}(M)$ is affine.

The reader should bear in mind that NS is of finite type; see [SGA6, XIII, Théorème 5.1, p. 650]. The proof of Proposition 16.3 is built up of the following results.

Lemma 16.4. Let $W$ be projective and connected. Let $W^{\prime}$ be the disjoint union of $n$ copies of $W$.

(i) Let $\mathfrak{S}_{n}$ be the constant group scheme associated with the symmetric group on $n$ letters. Then there exists a morphism of group schemes

$$
\sigma: \operatorname{Aut}_{k}\left(W^{\prime}\right) \longrightarrow \mathfrak{S}_{n}
$$

whose kernel is $\operatorname{Aut}_{k}(W)^{\times n}$. 


\section{J. P. DOS SANTOS}

(ii) In particular, if $\mathbf{A u t} \mathbf{t}_{k}(W)$ is affine, then $\mathbf{A u t}_{k}\left(W^{\prime}\right)$ is likewise.

Proof. First, we label $W_{1}, \ldots, W_{n}$ the connected components of $W^{\prime}$. Let $T \in$ Sch be connected and consider an automorphism of $T$-schemes $\varphi: W^{\prime} \times T \rightarrow W^{\prime} \times T$. It is clear that $W^{\prime} \times T$ is the disjoint union of the $W_{i} \times T$ and that each one of these is connected. Then $\varphi$ induces a permutation $\sigma(\varphi)$ of the set $\{1, \ldots, n\}$. It is easily verified that if $U \rightarrow T$ is a morphism from a connected scheme, then $\sigma\left(\varphi \times \times_{T} U\right)=\sigma(\varphi)$. This constructs the morphism of group functors $\sigma$. The kernel is easily determined and we conclude our proof of part (i). From this it follows that

$$
\left(\operatorname{Aut}_{k}(W)^{\times n}\right)^{0} \stackrel{\simeq}{\longrightarrow}\left(\operatorname{Aut}_{k}\left(W^{\prime}\right)\right)^{0} .
$$

Since each connected component of $\mathbf{A u t}_{k}\left(W^{\prime}\right)$ is a scheme isomorphic to $\mathbf{A u t}_{k}\left(W^{\prime}\right)^{0}$, it follows that $\mathbf{A u t}_{k}\left(W^{\prime}\right)$ is a disjoint union of affine schemes. Moreover, since $\sigma$ is surjective -in fact there exists a section $\mathfrak{S}_{n} \rightarrow \mathbf{A u t}_{k}\left(W^{\prime}\right)$-we conclude that $\mathbf{A u t}_{k}\left(W^{\prime}\right)$ is a disjoint union of a finite number of quasi-compact spaces. Consequently, $\mathbf{A u t}_{k}\left(W^{\prime}\right)$ is quasi-compact, so that its number of connected components is finite. A finite disjoint union of finitely many affine schemes is affine.

The proof of the following result is quite easy and we leave it to the reader.

Corollary 16.5. Let $M$ be a projective scheme. If $\mathbf{A u t}_{k}(V)$ is affine for each connected component $V$ of $M$, then $\operatorname{Aut}_{k}(M)$ is affine.

One important ingredient in verifying that an automorphism group scheme is affine relies on verifying that there are only finitely many connected components. Here is our result in this direction.

Lemma 16.6. Let $M$ be a projective scheme. Assume that its Néron-Severi group

$$
\operatorname{NS}(M)=\operatorname{Pic}(M) / \operatorname{Pic}^{0}(M)
$$

has rank one (as a finitely generated abelian group). Then $\mathbf{A u t}_{k}(M)$ is of finite type.

Proof. Given a very ample invertible sheaf $\mathcal{L}$ on $M$, we obtain an open immersion [Nit05, Exercise following Theorem 5.23, p. 133] $\gamma: \operatorname{Aut}_{k}(M) \rightarrow \mathbf{H i l b}_{M \times M}$ defined by associating with each automorphism its graph. (We use the very ample sheaf $\operatorname{pr}_{1}^{*} \mathcal{L} \otimes \operatorname{pr}_{2}^{*} \mathcal{L}$ on $M \times M$.) Write, as usual, $\mathbf{H i l b}_{M \times M}^{P}$ for the open subset of $\mathbf{H i l b}_{M \times M}$ associated with the polynomial $P \in \mathbb{Q}[m]$. If $a$ is a $k$-point of $\mathbf{A u t}_{k}(M)$, its image in $\mathbf{H i l b}_{M \times M}$ lies in $\mathbf{H i l b}_{M \times M}^{P}$, where $P(m)=\chi\left(\mathcal{L}^{m} \otimes a^{*} \mathcal{L}^{m}\right)$ for large $m$. From this, it follows that, if $a^{*} \mathcal{L}$ is $\tau$-equivalent to $\mathcal{L}$ (see [SGA6, XIII.4.4, p. 29]) for every $a \in \mathbf{A u t}_{k}(M)(k)$, then $\gamma$ sends $\mathbf{A u t}_{k}(M)$ into $\mathbf{H i l b}_{M \times M}^{P}$, where $P(m)=\chi\left(\mathcal{L}^{2 m}\right)$ for large $m$ (as explained in [SGA6, XIII, Théorème 4.6]). In particular, $\operatorname{Aut}_{k}(M)$ is quasi-projective.

Let $\mathbf{P i c}{ }_{M}^{\lambda}$ be a connected component of $\mathbf{P i c}{ }_{M}$ which generates the free part of $\mathrm{NS}(M)$ and which has a very ample class $\mathcal{L}$. We conclude that for each $a \in \mathbf{A u t}_{k}(M)(k)$, the sheaf $a^{*} \mathcal{L}$ is $\tau$-equivalent to $\mathcal{L}$.

Let us now fix a connected and projective scheme $V$. Denote the nilradical [EGA, I, 5.1] of $V$ by $\mathcal{N}$. For each $\mu$, let $V_{\mu}$ be the closed subscheme of $V$ cut out by $\mathcal{N}^{\mu}$.

Given any reduced $k$-scheme $T$, the closed subscheme $V_{\text {red }} \times T$ of $V \times T$ is reduced [EGA, $\mathrm{IV}_{2}$, Proposition 4.6.5, p. 69] and has the same underlying topological space as $V \times T$. Consequently, the nilradical of $\mathcal{O}_{V \times T}$ is the pullback of $\mathcal{N}$ by the obvious projection from $\mathcal{N} \times T$. Thus, for each $\varphi \in \operatorname{Aut}_{T}(V \times T)$, the associated isomorphism of rings

$$
\varphi^{\#}: \mathcal{O}_{V \times T, \varphi(x)} \longrightarrow \mathcal{O}_{V \times T, x}
$$




\section{Homotopy EXACT SEQUENCE}

maps $(\mathcal{N} \times T)_{\varphi(x)}$ onto $(\mathcal{N} \times T)_{x}$, and we can associate an automorphism of $V_{\mu} \times T$ with $\varphi$. In this way, we are able to define a morphism $\rho_{\mu}$ from the functor

$$
\mathbf{A}_{V}: \mathbf{R S c h}^{o} \longrightarrow \operatorname{Grp}, \quad T \longmapsto \operatorname{Aut}_{T}(V \times T)
$$

to the functor

$$
\mathbf{A}_{V_{\mu}}: \mathbf{R S c h}^{o} \longrightarrow \operatorname{Grp}, \quad T \longmapsto \operatorname{Aut}_{T}\left(V_{\mu} \times T\right) .
$$

Lemma 16.7. Let $\mu$ be such that $\mathcal{N}^{\mu}$ is of square zero. Then the functors

$$
\mathbf{R S c h}^{o} \longrightarrow \text { Set }, \quad T \longmapsto \operatorname{Ker} \rho_{\mu}(T)
$$

and

$$
\mathbf{R S c h}^{o} \longrightarrow \text { Set }, \quad T \longmapsto \operatorname{Der}_{\mathcal{O}_{T}}\left(\mathcal{O}_{V \times T}, \mathcal{N}^{\mu} \times T\right)
$$

are isomorphic, where $\mathcal{N}^{\mu} \times T$ is the pullback of $\mathcal{N}^{\mu}$ by the obvious projection.

Proof. The proof is in [SGA1, III, 5.1]. Let $T$ be reduced, and let

$$
\varphi: V \times T \longrightarrow V \times T
$$

be an isomorphism of $T$-schemes inducing the identity on $V_{\mu} \times T$. Then, $\varphi$ is determined by an automorphism of $\mathcal{O}_{T}$-algebras

$$
\varphi^{\#}: \mathcal{O}_{V \times T} \longrightarrow \mathcal{O}_{V \times T}
$$

such that

$$
\varphi^{\#}(a) \equiv a \quad \bmod \mathcal{N}^{\mu} \times T, \quad \forall a \in \mathcal{O}_{V \times T} .
$$

Thus, $\varphi$ is determined by the element

$$
\varphi^{\#}-\operatorname{id}_{\mathcal{O}_{V \times T}} \in \operatorname{Der}_{\mathcal{O}_{T}}\left(\mathcal{O}_{V \times T}, \mathcal{N}^{\mu} \times T\right) .
$$

Conversely, any element $\delta$ of the above group defines a morphism id $+\delta: \mathcal{O}_{V \times T} \rightarrow \mathcal{O}_{V \times T}$ of $\mathcal{O}_{T}$-algebras. Since this morphism is an isomorphism (the identity) modulo a nilpotent ideal, it follows that id $+\delta$ is an automorphism.

Let $\mu$ be as in the Lemma 16.7. Note that the functors $\mathbf{A}_{V}$ and $\mathbf{A}_{V_{\mu}}$ are represented by $\mathbf{A u t}_{k}(V)_{\text {red }}$ and $\mathbf{A u t}_{k}\left(V_{\mu}\right)_{\text {red }}$, respectively; as the category RSch possesses direct products, $\operatorname{Aut}_{k}(V)_{\text {red }}$ and $\mathbf{A u t}_{k}\left(V_{\mu}\right)_{\text {red }}$ are naturally group objects in $\mathbf{R S c h}$ and $\rho_{\mu}$ is a morphism of group objects. So we have a morphism of group schemes

$$
\rho_{\mu}: \operatorname{Aut}_{k}(V)_{\text {red }} \longrightarrow \mathbf{A u t}_{k}\left(V_{\mu}\right)_{\text {red }} .
$$

By [Nit05, Theorem 5.8] together with Lemma 16.7 and the equality

$$
\operatorname{Der}_{\mathcal{O}_{T}}\left(\mathcal{O}_{V \times T}, \mathcal{N}^{\mu} \times T\right)=\operatorname{Hom}_{V \times T}\left(\Omega_{V \times T / T}^{1}, \mathcal{N}^{\mu} \times T\right),
$$

the functor

$$
\mathbf{R S c h}^{o} \longrightarrow \text { Set }, \quad T \longmapsto \operatorname{Ker} \rho_{\mu}(T)
$$

is represented by an affine space $\mathbb{A}^{\ell}$. (We are not affirming that it is represented by $\mathbb{G}_{a}^{\ell}$ !) Hence, $\operatorname{Ker}\left(\rho_{\mu}\right)_{\text {red }}$ is affine and connected.

LEMma 16.8. We maintain the above notation. If $\left(\mathbf{A u t}_{k}\left(V_{\mu}\right)_{\mathrm{red}}\right)^{0}$ is affine, then $\mathbf{A u t}_{k}(V)^{0}$ is likewise.

Proof. Let $G=\operatorname{Aut}_{k}(V)_{\text {red }}$ and $H=\operatorname{Aut}_{k}\left(V_{\mu}\right)_{\text {red }}$. Let $K$ be the kernel of $\rho_{\mu}: G \rightarrow H$; above we showed that $K_{\text {red }}$ is connected and affine. By Lemmas 16.1 and 16.2, the kernel $K$ is affine, 


\section{J. P. DOS SANTOS}

connected, and of finite type. Due to the connectedness of $K$, the arrow $K \hookrightarrow G$ factors through $G^{0}$.

According to [SGA3, $\mathrm{VI}_{\mathrm{A}}, 3.2$ ], the quotient of the fppf sheaf $G^{0}$ by $K$ is representable by a group scheme $G^{0} / K$. Moreover, $\mathfrak{p}: G^{0} \rightarrow G^{0} / K$ is faithfully flat and of finite presentation [DG70, III.3.2.5, p. 328]. As $K$ is affine, [DG70, III.3.2.6, p. 329] shows that $\mathfrak{p}$ is affine. Now we only need to show that $G^{0} / K$ is affine.

Since $G^{0}$ is of finite type and $\mathfrak{p}$ is affine and faithfully flat, [BouAC, I.3.6, Proposition 11] guarantees that $G^{0} / K$ is locally of finite type. Since $G^{0}$ is quasi-compact and $\mathfrak{p}$ is surjective, $G^{0} / K$ is quasi-compact. As the induced morphism $G^{0} / K \rightarrow H^{0}$ is a monomorphism, it is a closed embedding by [DG70, II.5.5.1, p. 249]. It follows that $G^{0} / K$ is affine. The proof that $G^{0}$ is affine is then completed.

Since the topological spaces underlying $G^{0}$ and $\mathbf{A u t}_{k}(V)^{0}$ coincide, it follows from Lemmas 16.1 and 16.2 that $\mathbf{A u t}_{k}(V)^{0}$ is affine.

Proof of Proposition 16.3. Let $V$ be a connected component of $M$. Using [SGA6, XII, Théorème 1.1] (see also [SGA6, XIII, Lemme 4.3]), we know that

$$
\mathrm{NS}\left(V_{\text {red }}\right) /(\text { torsion }) \simeq \mathbb{Z} \quad \Longrightarrow \quad \mathrm{NS}(W) /(\text { torsion }) \simeq \mathbb{Z}
$$

for any nilpotent immersion $W \rightarrow V$. Using this fact, induction, Lemma 16.6, Lemma 16.8 and the affineness of $\mathbf{A u t}_{k}\left(V_{\text {red }}\right)$, we conclude that $\mathbf{A u t}_{k}(V)$ is affine. Corollary 16.5 now completes the proof.

\section{ACKNOWLEDGEMENTS}

This work profited greatly from P. H. Hai's ideas. In fact, it was by understanding some points of [Hai13] that the author developed the idea of considering the criteria defined in Section 4. Equally important was the encouraging interest P. H. Hai showed in some parts of the present work during a visit to Jussieu in 2011. We would also like to thank the referee for an exemplary job, B. Conrad for a very useful e-mail exchange, and H. Esnault for alerting us to overlapping work.

\section{REFERENCES}

AM94 M. Atiyah and I. MacDonald, Introduction to Commutative Algebra (Westview Press, 1994).

Ber74 P. Berthelot, Cohomologie cristalline des schémas de caractéristique $p>0$, Lecture Notes in Math., vol. 407 (Springer-Verlag, Berlin - New York, 1974); http://dx.doi.org/10.1007/ BFb0068636.

BLR90 S. Bosch, W. Lütkebohmert, and M. Raynaud, Néron models, Ergeb. Math. Grenzgeb. (3), vol. 21 (Springer-Verlag, Berlin, 1990); http://dx.doi.org/10.1007/978-3-642-51438-8.

BO78 P. Berthelot and A. Ogus, Notes on crystalline cohomology, (Princeton University Press, Princeton, N.J.; University of Tokyo Press, Tokyo, 1978).

BouAC N. Bourbaki, Éléments de mathématique, Algèbre commutative, Chapitres 1 à 4, Chapitres 5 à 7, Chapitres 8 et 9, and Chapitre 10 (Springer-Verlag, Berlin - Heidelberg, 2006-2007); http://dx.doi.org/10.1007/978-3-540-33976-2, http://dx.doi.org/ 10.1007/978-3-540-33979-3, http://dx.doi.org/10.1007/3-540-33980-9, and http:// dx.doi.org/10.1007/978-3-540-34395-0.

CLN85 C. Camacho and A. Lins Neto, Geometric Theory of Foliations (Birkhäuser Boston, Boston, MA, 1985); http://dx.doi.org/10.1007/978-1-4612-5292-4. 


\section{HOMOTOPY EXACT SEQUENCE}

DG70 M. Demazure and P. Gabriel, Groupes algébriques. Tome I: Géométrie algébrique, généralités, groupes commutatifs (Masson \& Cie, Éditeur, Paris; North-Holland Publishing Co., Amsterdam, 1970).

DM82 P. Deligne and J. Milne, Tannakian categories, Lecture Notes in Math., vol. 900, 101-228 (Springer-Verlag, Berlin-New York, 1982).

EGA A. Grothendieck and J. Dieudonné, Éléments de géométrie algébrique, Publ. Math. Inst. Hautes Études Sci. 4 (Chapter 0, 1-7, and I, 1-10), 8 (II, 1-8), 11 (Chapter 0, 8-13, and III, 1-5), 17 (III, 6-7), 20 (Chapter 0, 14-23, and IV, 1), 24 (IV, 2-7), 28 (IV, 8-15), and $\mathbf{3 2}$ (IV, 16-21), 1960-1967; available at http://www.numdam.org.

Ehr50 C. Ehresmann, Les connexions infinitésimales dans une espace fibré différentiable, Colloque de Topologie, Bruxelles, 1950.

EHV H. Esnault, H. H. Phùng, and E. Viehweg, On the homotopy exact sequence for Nori's fundamental group, arXiv:0908.0498.

Eke87 T. Ekedahl, Foliations and inseparable morphisms, Algebraic geometry, Bowdoin, 1985 (Brunswick, Maine, 1985), 139-149, Proc. Sympos. Pure Math., vol. 46 (2) (Amer. Math. Soc., Providence, RI, 1987); http://dx.doi.org/10.1090/pspum/046.2/927978.

ESB05 T. Ekedahl and N I. Shepherd-Barron, Tangent liftings of deformations in mixed characteristic, J. Algebra 291 (2005), no. 1, 108-128; http://dx.doi .org/10.1016/j · jalgebra. 2005.05.023.

GIT D. Mumford, J. Fogarty, and F. Kirwan, Geometric invariant theory, third ed., Ergeb. Math. Grenzgeb. (2), vol. 34 (Springer-Verlag, Berlin, 1994); http://dx.doi.org/10.1007/ 978-3-642-57916-5.

Hai13 P.H. Hai, Gauss-Manin stratification and stratified fundamental group schemes, Ann. Inst. Fourier (Grenoble) 63 (2013), no. 6, 2267-2285; http://dx.doi.org/10.5802/aif.2829.

Jan87 J. C. Jantzen, Representations of algebraic groups, Pure and Applied Mathematics, vol. 131 (Academic Press, Inc., Boston, MA, 1987).

Mat89 H. Matsumura, Commutative ring theory, second ed., Cambridge Stud. Adv. Math., vol. 8 (Cambridge Univ. Press, Cambridge, 1989); http://dx.doi.org/10.1017/CB09781139171762.

Miy87 Y. Miyaoka, Deformations of a Morphism along a Foliation and Applications, Algebraic Geometry, Bowdoin, 1985 (Brunswick, Maine, 1985), 245-268, Proc. Sympos. Pure Math., vol. 46 (1) (Amer. Math. Soc., Providence, RI, 1987); http://dx.doi.org/10.1090/pspum/046.1.

ML98 S. Mac Lane, Categories for the working mathematician, second ed., Grad. Texts in Math., vol. 5 (Springer-Verlag, New York, 1998); http://dx.doi.org/10.1007/978-1-4757-4721-8.

MM03 I. Moerdijk and J. Mrcun, Introduction to foliations and Lie groupoids, Cambridge Stud. Adv. Math., vol. 91 (Cambridge Univ. Press, Cambridge, 2003); http://dx.doi.org/10.1017/ CB09780511615450.

MO67 H. Matsumura and F. Oort, Representability of group functors, and automorphisms of algebraic schemes, Invent. Math. 4 (1967), 1-25; http://dx.doi.org/10.1007/BF01404578.

Mum70 D. Mumford, Abelian varieties, Tata Inst. Fund. Res. Stud. Math., vol. 5 (Oxford University Press, London, 1970).

Nit05 N. Nitsure, Construction of hilbert and quot schemes, Fundamental Algebraic Geometry, Grothendieck's FGA Explained, 105-137, Math. Surveys Monogr., vol. 123 (Amer. Math. Soc., Providence, RI, 2005).

Nor76 M. V. Nori, On the representations of the fundamental group, Compos. Math. 33 (1976), no. 1, 29-41; http://www . numdam.org/item?id=CM_1976__33_1_29_0.

dSan07 J.P.P. dos Santos, Fundamental group schemes for stratified sheaves, J. Algebra 317 (2007), no. 2,691-713; http://dx.doi.org/10.1016/j.jalgebra.2007.03.005.

Sch68 M. Schlessinger, Functors of Artin rings, Trans. Amer. Math. Soc. 130 (1968), 208-222; http: //dx.doi.org/10.2307/1994967. 


\section{J. P. DOS SANTOS}

Sei67 A. Seidenberg, Differential ideals in rings of finitely generated type, Amer. J. Math. 89 (1967), 22-42; http://www.jstor.org/stable/2373093.

SGA1 A. Grothendieck and M. Raynaud, Revêtements étales et groupe fondamental, Séminaire de géométrie algébrique du Bois Marie 1960-61, Updated and annotated reprint of the 1971 original, Doc. Math. (Paris), vol. 3 (Soc. Math. France, Paris, 2003).

SGA3 M. Artin, J.-E. Bertin, M. Demazure, A. Grothendieck, P. Gabriel, M. Raynaud, and J.-P. Serre, Jean-Pierre, Schémas en groupes, Séminaire de Géométrie Algébrique du Bois Marie 1962-64, Revised and annotated edition of the 1970 French original, edited by P. Gille and P. Polo, Doc. Math. (Paris), vol. 7 (Soc. Math. France, Paris, 2011).

SGA4 M. Artin, A. Grothendieck, and J.-L. Verdier, Théorie de topos et cohomologie étale des schémas I, II, III, Lecture Notes in Math. 269, 270, 305 (Springer-Verlag, 1971).

SGA6 P. Berthelot, A. Grothendieck, and L. Illusie, Théorie des intersections et Théorème de RiemannRoch, Lecture Notes in Math. 225 (Springer-Verlag, 1971).

SR72 N. Saavedra Rivano, Catégories Tannakiennes, Lecture Notes in Math., vol. 265, Springer-Verlag, Berlin - New York, 1972; http://dx.doi.org/10.1007/BFb0059108.

TDTE A. Grothendieck, Technique de descente et théorèmes d'existence en géométrie algébrique, Séminaire Bourbaki 5 (I: Exp. no. 190, 299-327 and II: Exp. no. 195, 369-390), 6 (III: Exp. no. 212, 99-118 and IV: Exp. no. 221, 249-276), 7 (V: Exp. no. 232, 143-161 and VI: Exp. no. 236, 221-243), 1959-1962; available at http://www. numdam.org.

Tei93 B. Teissier, Résultats récents sur l'approximation des morphismes en algèbre commutative, (d'après André, Artin, Popescu et Spivakovsky), Astérisque 227 (1995), Exp. No. 784, 4, 259-282.

Wat79 W. Waterhouse, Introduction to affine group schemes, Graduate Texts in Math., vol. 66, SpringerVerlag, New York - Berlin, 1979; http://dx.doi.org/10.1007/978-1-4612-6217-6.

Zha13 L. Zhang, The homotopy sequence of the algebraic fundamental group, Int. Math. Res. Not. IMRN 2014 (2014), no. 22, 6155-6174; http://dx.doi.org/10.1093/imrn/rnt163.

João Pedro dos Santos joao_pedro.dos_santos@yahoo.com

Institut de Mathématiques de Jussieu, Paris Rive Gauche, 4, Place Jussieu, 75005 Paris, France 